Existência e concentração de soluções para sistemas elípticos com condição de Neumann

Marcos Tadeu de Oliveira Pimenta 

SERVIÇO DE PÓS-GRADUAÇÃO DO ICMC-USP

Data de Depósito: 25 de janeiro de 2008

Assinatura:

\section{Existência e concentração de soluções para sistemas elípticos com condição de Neumann ${ }^{1}$}

\section{Marcos Tadeu de Oliveira Pimenta}

\section{Orientador: Prof. Dr. Sérgio Henrique Monari Soares}

Dissertação apresentada ao Instituto de Ciências Matemáticas e de Computação - ICMC/USP, como parte dos requisitos para obtenção do título de Mestre em Matemática.

\section{USP - São Carlos}

Março/2008

\footnotetext{
${ }^{1}$ Projeto financiado pela FAPESP
} 

Aos meus pais

Aloísio e Ana, ao meu irmão João Paulo e à minha esposa Lane. 



\section{Agradecimentos}

Agradeço primeiramente a Deus pela graça imensa que sempre me concedeu nos momentos em que a ele apelei e também nos momentos, embora poucos, em que dele me esqueci. A toda a minha família, sobretudo a meus pais, que sempre me proporcionaram todas as oportunidades de estudos que estavam ao alcance deles e também por terem pensado única e exclusivamente no meu melhor. Ao meu incansável pai Aloísio, pelo exemplo de trabalho, hombridade e responsabilidade para com a família, na sua batalha pela vida em todos os momentos literais de sol ou chuva, investindo sempre na educação dos filhos. A minha mãe Ana, exemplo maior da minha vida, lutadora que é e sempre foi, constantemente apoiando-me em todas as decisões que tomei, por sua solidariedade, doação, responsabilidade e dedicação integral e irrestrita à família, principalmente aos filhos, para que buscassem a realização de seus sonhos. Ao meu irmão João Paulo, que sempre foi o meu companheiro de todas as horas e com quem dividi os momentos mais importantes da minha vida, pelo seu espírito protetor e zeloso para com o seu irmão mais novo, transmitindo a segurança necessária para que eu pudesse alçar vôos mais altos na minha formação.

Agradeço a minha esposa Lane pelo apoio incondicional e pela tranqüilidade que sempre me transmitiu ao longo desta caminhada e pela forma amorosa, carinhosa, zelosa e companheira como sempre me tratou, por todas as palavras de carinho e pela paciência que teve e continua a ter, enquanto suporta os $450 \mathrm{~km}$ que nos separam atualmente, mas se Deus quiser, por pouco tempo. Agradeço também à família da minha esposa que, desde que nos casamos, passou a ser minha também. Ao meu sogro Moacyr e a minha sogra Vera, por sempre terem me tratado e acolhido como a um filho, proporcionando-me o incentivo necessário para que eu continuasse a trilhar o caminho que escolhi. Às minhas cunhadas Talita e Vanessa, com as quais cultivo excelente relacionamento, pelos momentos de descontração, tão importantes em dias de trabalho árduo.

Agradeço também a todos os funcionários, professores e colegas da Escola Estadual Prof ${ }^{a}$ Maria Luiza Formozinho Ribeiro de Presidente Prudente, onde cursei os Ensinos Fundamental 
e Médio, muito especialmente à Professora de Matemática, Miriam Luiza, que sempre me incentivou com palavras de orientação e apoio.

Agradeço a todos os funcionários e professores do Departamento de Matemática da FCT UNESP de Presidente Prudente, onde conclui meus estudos de graduação, especialmente ao Prof ${ }^{\circ}$ Dr. Marcelo Messias, pela excelente orientação que me ofereceu durante a minha iniciação científica. A todos os inesquecíveis bons amigos que fiz por lá, que comigo dividiram alguns dos momentos mais engraçados e memoráveis da minha vida. Agradeço a todos os funcionários e professores do ICMC - USP, sobretudo ao Prof ${ }^{\circ}$ Dr. Sérgio Henrique Monari Soares, que me conduziu e orientou nesse trabalho de uma forma absolutamente exemplar, séria e atenciosa, me oferecendo assim um exemplo de profissional a seguir. Agradeço a todos os amigos que fiz aqui no ICMC, sobre tudo aos companheiros de turma. A todos os companheiros da sala 4009 (Gil, Castilho, Claudinei, Leitão, Lucas e Wescley) e 4232. Aos amigos Hartmann, Ubarana, Edinho, Eduardo, Thaís, Yuri e todos os companheiros de república Juliano, Cláudio, Fabrício, Dusse e Alex, pelos momentos de descontração e alegria.

Por fim, porém não menos importante, agradeço à Fapesp pelo fomento. 
Estudamos a seguinte classe de sistemas elípticos

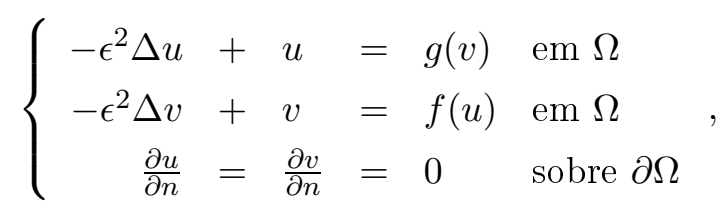

onde $\Omega \subset \mathbb{R}^{N}$ é um domínio limitado, com bordo regular e $N \geq 3$. As não linearidades $f$ e $g$ são funções com crescimento superlinear e subcrítico no infinito. Estudamos resultados sobre existência de soluções que se concentram, quando $\epsilon$ tende a zero, em um ponto da fronteira que maximiza a sua curvatura média. Para isso utilizamos um resultado abstrato sobre existência de pontos críticos para funcionais fortemente indefinidos. 

We study the following elliptic system

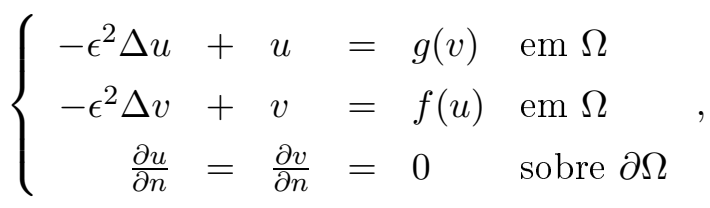

when $\Omega \in \mathbb{R}^{N}$ is a smooth bounded domain, $N \geq 3$ and $f$ and $g$ are nonlinearities having superlinear and subcritical growth at infinity. We study an abstract result about existence of critical points of strongly indefinite functionals to study the existence of solutions with concentrate, as $\epsilon$ goes to zero, at a point of the boundary which maximizes the mean curvature of the boundary. 



\section{Índice de Notações}

$\begin{array}{ll}E^{+}\left(E^{-}\right) & \text {Auto espaço positivo (negativo) de um operador } \\ P_{V} & \text { Projeção ortogonal do subespaço } V \text { sobre o espaço } E \\ \operatorname{dim}_{W} V & \text { Dimensão relativa de } V \text { com respeito a } W \\ m_{\left(E^{+}, E^{-}\right)}(x ; f) & \text { Índice de Morse relativo de } x \text { com respeito a fatoração } E=E^{+} \oplus E^{-} \\ B_{r}(x) & \text { Bola aberta de centro em } x \text { e raio } r>0 \\ B_{r} & \text { Bola aberta de centro em } 0 \text { e raio } r>0 \\ \partial \Omega & \text { Fronteira do conjunto } \Omega \subset \mathbb{R}^{N} \\ i n t(\Omega) & \text { Conjunto dos pontos interiores de } \Omega \\ K_{c} & \text { Conjunto dos pontos críticos em } E \text { no nível } c \\ f^{c} & \text { Conjunto dos } x \in E \text { tais que } f(x) \leq c \\ C^{m}(E) & \text { Espaço das funções } m \text { vezes continuamente diferenciáveis sobre } \mathbb{R} \\ \|f\|_{L^{p}(\Omega)} & \|f\|_{L^{p}(\Omega)}=\left(\int_{\Omega}|f|^{p}\right)^{\frac{1}{p}} \\ L^{p}(\Omega) & \text { Conjunto das funções Lebesgue mensuráveis com }|\cdot|_{L^{p}}<\infty \\ H^{1}(\Omega) & \text { Espaço de Sobolev } W^{1,2}(\Omega) \\ p^{*} & p^{*}=\frac{p N}{N-p} \\ p^{\prime} & p^{\prime}=\frac{p}{p-1} \\ s u p p(\varphi) & \text { Fecho do conjunto }\{x \in E ; \varphi(x)=0\} \\ \nabla \Phi & \text { Vetor gradiente de } \Phi\end{array}$





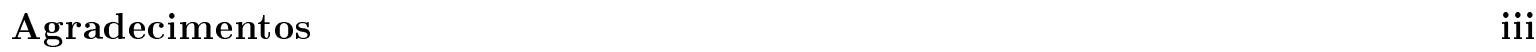

Resumo $v$ v

$\begin{array}{lll}\text { Abstract } & \text { vii }\end{array}$

$\begin{array}{ll}\text { Îndice de Notações } & \text { ix }\end{array}$

$\begin{array}{ll}\text { Introdução } & 1\end{array}$

1 Estimativas de Morse $\quad 5$

1.1 Introdução . . . . . . . . . . . . . . . . . . . . . 5 5

1.2 Definições e resultados preliminares . . . . . . . . . . . . . . 5 5

1.3 Existência de pontos críticos e estimativa de Morse . . . . . . . . . . . . . . 7

2 Existência e concentração de soluções para um sistema elíptico 15

2.1 Introdução . . . . . . . . . . . . . . . . . . . . . . . . . . . . . . 15

2.2 Estimativas a priori de soluções . . . . . . . . . . . . . . . . . . . 16

2.3 Forma das soluções positivas . . . . . . . . . . . . . . . . . . 30

2.4 Estimativas de Energia . . . . . . . . . . . . . . . . . . . . . . 33

2.5 Resultados de Concentração . . . . . . . . . . . . . . . . . . . . . . 40

2.6 Localização dos Pontos de Máximo . . . . . . . . . . . . . . . . 45

$\begin{array}{ll}\text { Referências Bibliográficas } & 53\end{array}$ 



\section{Introdução}

Nesse trabalho estudamos a questão de existência e algumas características das soluções do seguinte sistema elíptico semilinear:

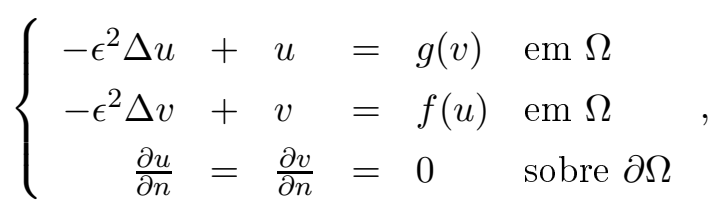

onde $\Omega \in \mathbb{R}^{N}$ é um domínio limitado com fronteira suave e $N \geq 3$.

Trata-se de um sistema Hamiltoniano singularmente perturbado, onde estudamos o comportamento assintótico das soluções não triviais, quando o parâmetro $\epsilon$ tende a 0 .

Quando considera-se o problema escalar $-\epsilon^{2} \Delta u+u=f(u)$ em $\Omega$, com condição de Neumann na fronteira, observa-se que as soluções $u_{\epsilon}$ apresentam a formação de um pico cada vez mais esticado em torno do seu ponto de máximo $x_{\epsilon}$, a medida em que $\epsilon$ converge para 0 . Isto parece bastante natural se considerarmos o reescalonamento das soluções dado por $\tilde{u}_{\epsilon}(x)=u_{\epsilon}\left(\epsilon x+x_{\epsilon}\right)$, pois $\tilde{u}_{\epsilon}$ satisfaz a equação $-\Delta \tilde{u}_{\epsilon}+\tilde{u}_{\epsilon}=f\left(\tilde{u}_{\epsilon}\right)$ no domínio expandido $\Omega_{j}=\frac{1}{\epsilon}\left(\Omega-x_{\epsilon}\right)$ e converge em $C_{l o c}^{2}$ para $u$ solução da equação limite $-\Delta u+u=f(u)$ em $\omega$, onde $\omega=\mathbb{R}^{N}$ ou um semiplano. Em qualquer dos casos, sob algumas condições sobre a não linearidade $f$, a equação limite apresenta uma solução que tem um ponto de máximo em 0 e é exponencialmente decrescente em qualquer direção radial. Dessa forma, podemos inferir um comportamento semelhante para $\tilde{u}_{\epsilon}$ e, conseqüentemente para $u$. Assim, uma pergunta que surge naturalmente é se tal resultado é verdadeiro para o sistema (1) e onde se localizam esses pontos de máximo das funções $u_{\epsilon}$ e $v_{\epsilon}$ que compõem a solução.

Graficamente, procuramos soluções que apresentem o seguinte comportamento. 


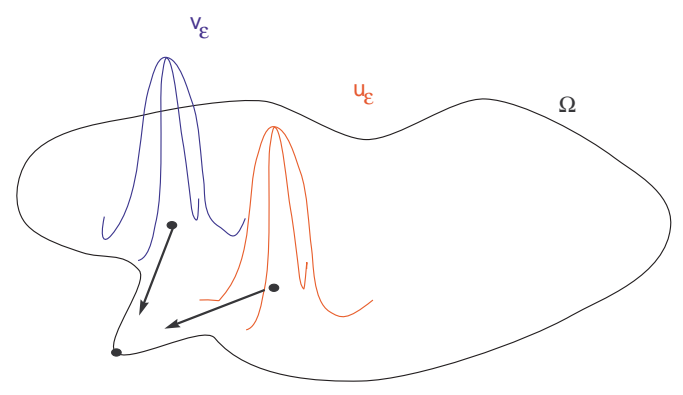

No Capítulo 1, vamos apresentar um resultado abstrato sobre a existência de pontos críticos para funcionais da forma $f(x)=\frac{1}{2}(L x, x)+h(x)$, onde $x \in H, H$ é um espaço de Hilbert, $L$ é um operador invertível autoadjunto e $h \in C^{2}$ com $h^{\prime}$ compacto. Este teorema fornecerá ainda uma estimativa para o índice de Morse relativo dos pontos críticos que será de fundamental importância no Capítulo 2, onde quase todos os resultados dependem de tal limitação uniforme.

No Capítulo 2 usaremos os resultados estudados no Capítulo 1 para tratar das questões de existência, do comportamento assintótico e localização dos pontos de máximo das soluções do problema, quando $\epsilon \rightarrow 0$. Consideraremos o seguinte conjunto de hipóteses sobre as não linearidades $f$ e $g$ :

(H) $f, g \in C^{1}(\mathbb{R}), f(0)=g(0)=f^{\prime}(0)=g^{\prime}(0)=0$ e existem $l_{1}, l_{2}>0$ e $p, q>2$ tais que $\frac{1}{p}+\frac{1}{q}>\frac{N-2}{N} \mathrm{e}$

$$
\lim _{|s| \rightarrow \infty} \frac{f^{\prime}(s)}{|s|^{p-2}}=l_{1} \quad \text { e } \quad \lim _{|s| \rightarrow \infty} \frac{g^{\prime}(s)}{|s|^{q-2}}=l_{2} .
$$

Além disso, para algum $\delta>0$ e todo $s \in \mathbb{R}, s \neq 0$,

$$
f(s) s \geq(2+\delta) F(s)>0 \quad \text { e } \quad f^{2}(s) \leq 2 f^{\prime}(s) F(s)
$$

e igualmente para $g$.

Sob essas hipóteses, vamos verificar que o funcional energia associado ao sistema (1), satisfaz as condições geométricas do teorema fundamental do Capítulo 1, que fornecerá soluções com índice de Morse limitados. Vamos utilizar as estimativas de Morse e o conjunto de hipóteses $(\mathrm{H})$ para mostrar que as soluções obtidas são não constantes, para valores de $\epsilon$ suficientemente pequenos. Mostraremos também que a limitação uniforme no índice de Morse implicará numa limitação uniforme na norma $L^{\infty}$ das soluções. Dessa forma, o funcional energia estará bem definido sobre o espaço $E=H^{1}(\Omega) \times H^{1}(\Omega)$. Utilizaremos uma estimativa superior para a energia do sistema para mostrar que as funções $u_{\epsilon}$ e $v_{\epsilon}$ que formam a solução do sistema (1), atingem seus valores máximos em um único e comum ponto da fronteira de $\Omega$, para valores de $\epsilon$ suficientemente pequenos. Por fim, utilizaremos também uma estimativa inferior da energia do sistema em termos da curvatura média da fronteira, para provar que este ponto onde $u_{\epsilon} \mathrm{e}$ $v_{\epsilon}$ atingem seus valores máximos, maximiza a curvatura média da fronteira. 
Para estimar o índice e Morse, seguiremos o trabalho [1], enquanto que para a existência e características das soluções, seguiremos o trabalho [8]. Na última seção, onde se prova a localização dos pontos de máximo, foi seguido o trabalho [6].

Caso o leitor deseje, a leitura do texto pode ser iniciada pelo Capítulo 2, necessitando para isso, apenas assumir o enunciado do Teorema 1.3.2. 



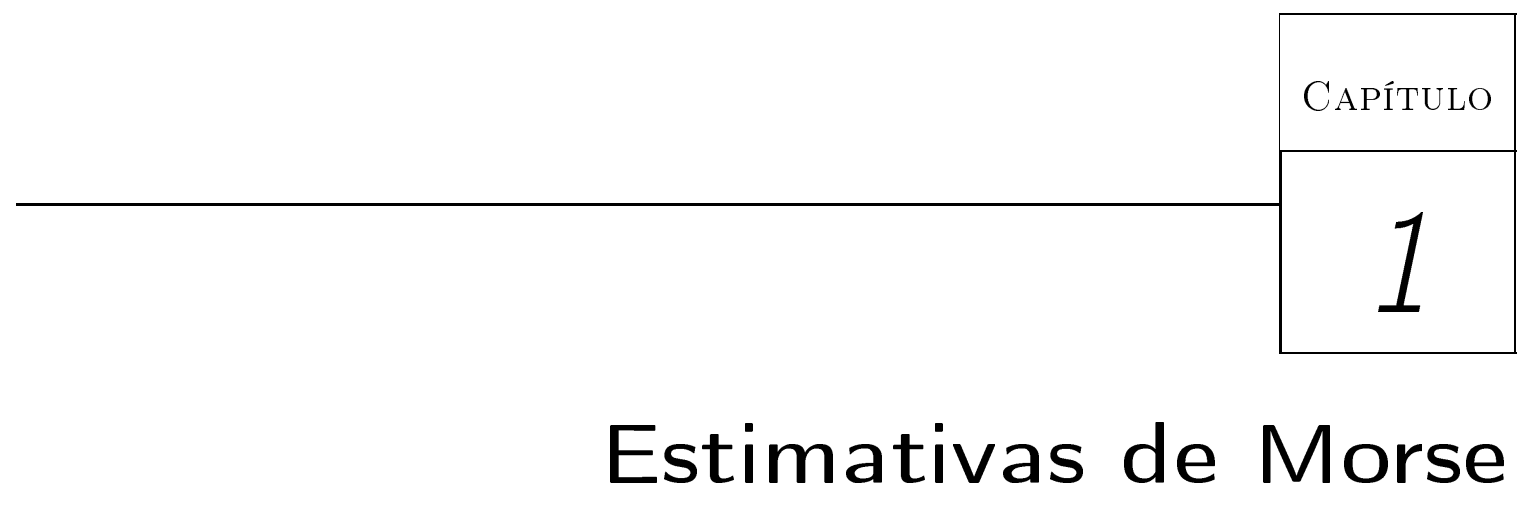

\subsection{Introdução}

Nesse capítulo estudaremos uma extensão do teorema de linking de Benci-Rabinowitz, devido a Abbondandolo, Felmer e Molina, para funcionais fortemente indefinidos satisfazendo a condição de Palais-Smale. Esta nos fornecerá pontos críticos do funcional estudado, bem como uma limitação superior do índice de Morse.

A parte de existência do resultado principal desse capítulo será usado para fornecer as soluções do sistema elíptico que estudaremos no Capítulo 2, que mostraremos que satisfazem um fenômeno de concentração. Para isso, será de fundamental importância uma estimativa superior no índice de Morse relativo, que por sua vez nos fornecerá uma estimativa superior na norma $L^{\infty}$ de tais soluções. Isto nos possibilitará trabalhar com o espaço $E=H^{1}(\Omega) \times H^{1}(\Omega)$. Por último, utilizaremos a estimativa para mostrar que as soluções obtidas são não constantes, uma vez que estas, possuem índice de Morse crescentes.

Toda a teoria e a forma de argumentação presentes nesse capítulo, seguiu o trabalho [1].

\subsection{Definições e resultados preliminares}

Seja $E$ espaço de Hilbert com produto interno $(\cdot, \cdot)$. Consideraremos funcionais $f: E \rightarrow \mathbb{R}$ escritos na seguinte forma:

$$
f(u)=\frac{1}{2}(L u, u)+h(u)
$$


onde $L: E \rightarrow E$ é um operador autoadjunto invertível, $h \in C^{2}(E)$ e $h^{\prime}$ compacto. Nosso interesse se voltará para o caso fortemente indefinido, ou seja, quando os autoespaços positivo e negativo de $L$ são de dimensão infinita.

Denotemos por $E^{+}$e $E^{-}$, respectivamente, os autoespaços positivo e negativo de $L$ e, dado $V \subset E$ subespaço fechado, $P_{V}$ a projeção ortogonal de $V$ sobre $E$.

A seguir, enunciaremos uma série de observações e definições que serão utilizadas posteriormente na demonstração de resultados fundamentais deste capítulo.

Definição 1.2.1. Dados $V, W \subset E$ subespaços fechados, dizemos que $V$ e $W$ são comensuráveis se $P_{V}{ } P_{W}$ e $P_{W}{ }^{\perp} P_{V}$ são operadores compactos. Nesse caso, a dimensão relativa de $V$ com respeito a $W$ está bem definida como o inteiro:

$$
\operatorname{dim}_{W} V=\operatorname{dim}\left(V \cap W^{\perp}\right)-\operatorname{dim}\left(V^{\perp} \cap W\right) .
$$

Observe que a dimensão relativa é diferente da dimensão algébrica de um subespaço, pois a primeira pode inclusive assumir valores inteiros negativos.

Note que a comensurabilidade de $V$ e $W$ implica que as duas parcelas da soma acima são finitas. De fato, se por exemplo $\operatorname{dim} V \cap W^{\perp}=\infty$, então a bola unitária restrita a $V \cap W^{\perp}$ não é compacta. Porém o operador $P_{W} P_{V}$ leva a bola unitária nela própria. Assim este operador não seria compacto.

Dados $V, W$ e $Z$ espaços comensuráveis, temos as seguintes propriedades:

- $\operatorname{dim}_{W} V=-\operatorname{dim}_{V} W$;

- $\operatorname{dim}_{Z} V=\operatorname{dim}_{W} V+\operatorname{dim}_{Z} W$;

- $V^{\perp}$ e $W^{\perp}$ são comensuráveis e $\operatorname{dim}_{V^{\perp}} W^{\perp}=-\operatorname{dim}_{V} W$;

Definição 1.2.2. Um operador $T: E \rightarrow E$ é de Fredholm se a dimensão do núcleo e a codimensão da imagem são finitos. Definimos ainda o índice de $T$ como a diferença entre a dimensão do núcleo e a codimensão da imagem.

Proposição 1.2.3. Seja $T$ um operador de Fredholm autoadjunto. Se $K$ é um operador autoadjunto compacto, os autoespaços positivo (negativo) de $T$ e $T+K$ são comensuráveis. Reciprocamente, se $T$ é invertivel e autoadjunto com autoespaço positivo (negativo) $E^{+}\left(E^{-}\right)$ e $E=W^{+} \oplus W^{-}$, onde $W^{+}\left(W^{-}\right)$é comensurável com $E^{+}\left(E^{-}\right)$, então existe um operador autoadjunto invertivel $M$ cujo autoespaço positivo (negativo) é $W^{+}\left(W^{-}\right)$. Além disso, $M-T$ é compacto.

Observação 1.2.4. Sejam $X$ e $Y$ dois subespaços fechados comensuráveis de $E$. Se $\operatorname{dim}_{Y} X>0$, então existe $T: E \rightarrow E \operatorname{com} T=I+K$ invertível tal que $T(X)=Y \oplus Y_{r}$ onde $\operatorname{dim} Y_{r}<\infty$ e $Y_{r} \neq \emptyset$.

Definição 1.2.5. Seja $x$ um ponto crítico de $f$ onde $f$ é duas vezes diferenciável. Seja $E=V^{+} \oplus V^{-} \oplus V^{0}$ decomposição de $E$ nos autoespaços positivo, negativo e nulo de 
$f^{\prime \prime}(x)=L+K$ onde $K$ é compacto autoadjunto. $\mathrm{O}$ índice de Morse relativo de $x$ com respeito à fatoração $E^{+} \oplus E^{-}$de $E$ é o inteiro:

$$
m_{\left(E^{+}, E^{-}\right)}(x ; f)=\operatorname{dim}_{E^{-}} V^{-} .
$$

Observação 1.2.6. Note que $m_{\left(E^{+}, E^{-}\right)}(x ; f)$ está bem definido, pois como $f^{\prime \prime}=L+K$, onde $K$ é compacto, então pela Proposição $1.2 .3, V^{-}$e $E^{-}$são comensuráveis.

Proposição 1.2.7. Seja $\left(K_{n}\right)$ uma sequência de operadores compactos autoadjuntos que converge para $K$. Seja $\left(f_{n}\right)$ uma sequência $C^{2}$ em $x$ e suponha que $f_{n}^{\prime \prime}(x)=L+K_{n}$ e $f^{\prime \prime}(x)=L+K$. Então:

$$
m_{\left(E^{+}, E^{-}\right)}(x ; f) \leq \liminf _{n \rightarrow \infty} m_{\left(E^{+}, E^{-}\right)}\left(x ; f_{n}\right)
$$

\subsection{Existência de pontos críticos e estimativa de Morse}

A seguir apresentamos o resultado principal deste capítulo que será de fundamental importância no Capítulo 2.

Definição 1.3.1. Seja $E$ um espaço de Banach e $f$ um funcional $C^{1}$ sobre $E$. Dizemos que $f$ satisfaz a condição de Palais Smale no nível $c \in \mathbb{R}$ se qualquer sequência $\left(u_{n}\right) \subset E$ tal que $f\left(u_{n}\right) \rightarrow c$ e $f^{\prime}\left(u_{n}\right) \rightarrow 0$ tem uma subsequência convergente. Neste caso dizemos que $f$ satisfaz a condição $(P S)_{c}$. Dizemos ainda que $f$ satisfaz a condição de Palais Smale se qualquer sequência $\left(u_{n}\right) \subset E$ tal que $\left|f\left(u_{n}\right)\right|$ é limitada e $f^{\prime}\left(u_{n}\right) \rightarrow 0$ tem uma subsequência convergente. Nesse caso dizemos simplesmente que $f$ satisfaz $(P S)$.

Teorema 1.3.2. Seja $E=W^{+} \oplus W^{-}$uma decomposição de $E$ onde $W^{+}$é comensurável com $E^{+}$e $W^{-}$com $E^{-}$. Seja $e \in \partial B_{1} \cap W^{+} e$

$$
S=\partial B_{\rho} \cap W^{+} \quad e \quad Q=B_{s} \cap W^{-} \oplus[0, r] e,
$$

onde $r>\rho>0$ e $s>0$. Denotando por $\partial Q$ a fronteira de $Q$ em $W^{-} \oplus \mathbb{R}$, suponha que existam reais $\alpha<\beta$ tais que:

$$
\sup _{\partial Q} f<\alpha<\inf _{S} f \quad e \quad \sup _{Q} f<\beta .
$$

Suponha ainda que $f$ satisfaça $(P S)_{c}$ para todo $c \in[\alpha, \beta]$. Então $f$ tem um ponto crítico $x$ tal que $\alpha \leq f(x) \leq \beta$. Além disso, se $f \in C^{2}(E)$ temos a seguinte estimativa para o indice de Morse relativo:

$$
m_{\left(E^{+}, E^{-}\right)}(x ; f) \leq \operatorname{dim}_{E^{-}} W^{-}+1
$$

Graficamente, podemos representar as hipóteses geométricas do teorema acima da seguinte forma 


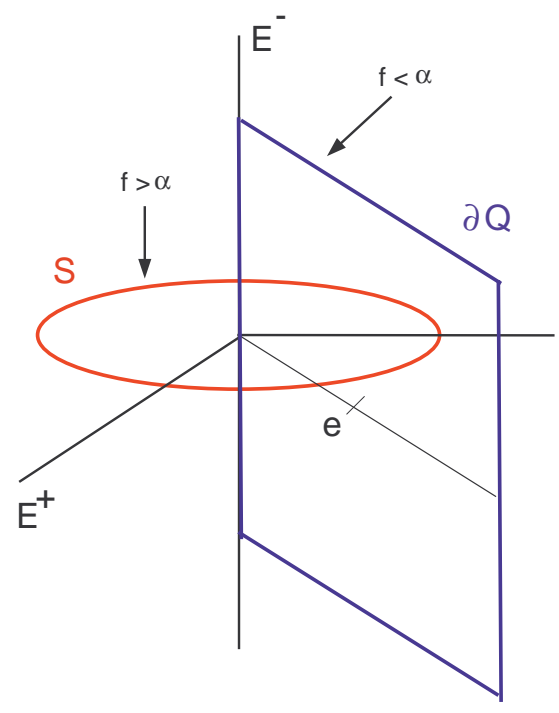

Supondo verdadeira a existência do valor crítico $c$ enunciado no Teorema 1.3.2, será mostrado que podemos supor, sem perda de generalidade, que todos os pontos críticos no nível $c$ são não degenerados.

Teorema 1.3.3. Sob as hipóteses do Teorema (1.3.2) e supondo que todos os pontos críticos no nivel c são não-degenerados, existe um ponto crítico $u_{0}$ tal que $f\left(u_{0}\right)=$ ce $m_{\left(W^{+}, W^{-}\right)}\left(u_{0} ; f\right) \leq$ 1.

Deixaremos a demonstração desse resultado, que é bastante técnica, para o final deste capítulo.

Definição 1.3.4. Seja $U(x)$ uma vizinhança aberta de $x \in E$. Dizemos que $f \in C^{2}(U(x))$ é uma função de Morse em $U(x)$ se as seguintes condições valem:

- $f(x)$ satisfaz (PS) em $U(x)$;

- $f$ tem somente pontos críticos não-degenerados em $U(x)$.

Observação 1.3.5. Seja $x$ um ponto crítico degenerado de $f \in C^{2}(U(X))$. Suponha que $f$ satisfaz (PS) em $U(x)$ e $f^{\prime \prime}(x)=T+K$ onde $T$ é invertível autoadjunto e $K$ é compacto autoadjunto. Então o Teorema de Marino-Prodi (que pode ser visto em [5]) afirma que existe uma sequência $\left(f_{n}\right)$ de funções de Morse, em $U(x)$ tal que: $f_{n}=f$ em $E \backslash B_{\frac{1}{n}}(x) \forall n \in \mathbb{N}$ e também $f_{n} \rightarrow f$ em $C^{2}(U(x))$.

Observação 1.3.6. Supondo verdadeiro o Teorema (1.3.3), para provar a estimativa de Morse contida no Teorema (1.3.2), não há perda de generalidade em supor que todos os pontos críticos do nível $c$ são não-degenerados.

De fato, seja $u_{0}$ um ponto crítico degenerado obtido no Teorema(1.3.2). Então existe uma sequência de pontos críticos $\left\{u_{n}\right\} \operatorname{com} u_{n} \rightarrow u_{0}$. Note que $f^{\prime \prime}\left(u_{0}\right)=T+K$ e como $f$ satisfaz $(P S)_{c}$ com $c \in[\alpha, \beta]$ e é contínua, $f$ satisfaz $(P S)$ em $U\left(u_{0}\right)$, para alguma vizinhança de $u_{0}$. 
Logo a Observação (1.3.5) garante a existência de uma sequência de funções de Morse $\left\{f_{n}\right\}$ tais que $f_{n}=f$ em $E \backslash B\left(u_{0}, \frac{1}{n}\right) \forall n \in \mathbb{N}$ e também $f_{n} \rightarrow f$ em $C^{2}\left(U\left(u_{0}\right)\right)$.

Para $n$ suficientemente grande, $f_{n}$ satisfaz as hipóteses do Teorema (1.3.2), logo, sem perda de generalidade, podemos considerar cada elemento $u_{n}$ da sequência como sendo um ponto crítico min-max no nível $c_{n}$ vindo da parte de existência do Teorema (1.3.2). Como cada $f_{n}$ é uma função de Morse então $u_{n}$ é ponto crítico não-degenerado de $f_{n}$, dessa forma, aplicando o Teorema (1.3.3) obtemos que

$$
m_{\left(W^{+}, W^{-}\right)}\left(u_{n}, f_{n}\right) \leq 1
$$

para todo $n$ suficientemente grande. Segue então da Proposição 1.2.7 que

$$
m_{\left(W^{+}, W^{-}\right)}\left(u_{n}, f\right) \leq 1 .
$$

Conseqüentemente $m_{\left(W^{+}, W^{-}\right)}\left(u_{0}, f\right) \leq 1$.

Vamos enunciar agora um resultado que é fundamental para a prova do Teorema 1.3.2.

Lema 1.3.7 (Lema da Deformação). Suponha que $f \in C^{1}(E)$ e satisfaça $(P S)_{c}$ para $\alpha \leq c \leq \beta$. Seja $K_{c}=\left\{u \in E ; f(u)=c\right.$ e $\left.f^{\prime}(u)=0\right\}$. Se c não for um valor crítico de $f$, então dado $\bar{\epsilon}>0$, existe $\epsilon \in(0, \bar{\epsilon})$ e $\eta \in C([0,1] \times E, E)$, tal que

- $\eta\left(1, f^{c+\epsilon}\right) \subset f^{c-\epsilon}$

- $\eta(1, u)=u$ se $f(u) \notin[c-\bar{\epsilon}, c+\bar{\epsilon}]$,

onde $f^{c}=\{u \in E ; f(u) \leq c\}$.

Graficamente, podemos representar a atuação da deformação $\eta$ no espaço $E$ da seguinte forma.

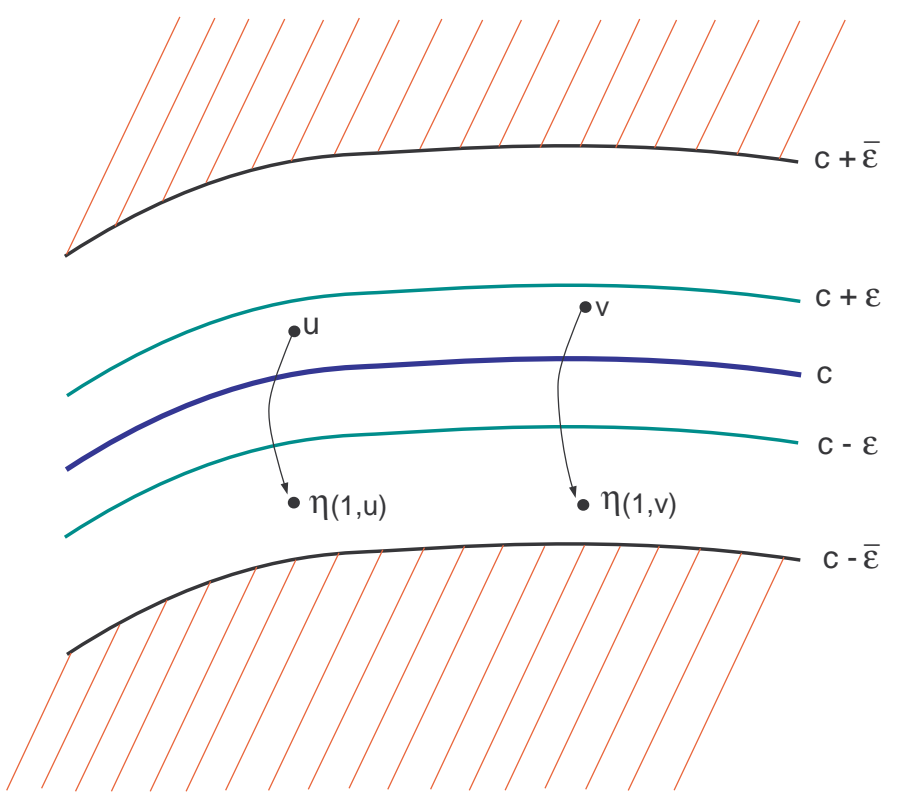


Apresentamos agora a prova do Teorema 1.3.2

Demonstração. Mostremos inicialmente que não há perda de generalidade em supor que $W^{+}=E^{+}$e $W^{-}=E^{-}$. De fato, como o operador $L$ é autoadjunto e invertível, pela Proposição 1.2.3, existe um operador autoadjunto invertível $M$ cujo autoespaço positivo (negativo) é $W^{+}\left(W^{-}\right)$e tal que $(L-M)$ é compacto. Então $f^{\prime \prime}(x)=L+K=M+$ $[(L-M)+K]$. Supondo então que a estimativa do índice de Morse relativo valha para $W^{+}=E^{+}$e $W^{-}=E^{-}$, teremos que $m_{\left(W^{+}, W^{-}\right)}(x, f) \leq 1$. Portanto, para o caso geral, denotando por $V^{+}\left(V^{-}\right)$o autoespaço positivo (negativo) de $f^{\prime \prime}(x)$,

$$
m_{\left(E^{+}, E^{-}\right)}(x, f)=\operatorname{dim}_{E^{-}} V^{-}=\operatorname{dim}_{W^{-}} V^{-}+\operatorname{dim}_{E^{-}} W^{-} \leq \operatorname{dim}_{E^{-}} W^{-}+1 .
$$

Seja $\Gamma=\left\{g \in C(E, E) ; g\right.$ satisfaz $\Gamma_{1}$ e $\left.\Gamma_{2}\right\}$

$\left(\Gamma_{1}\right) g(u)=u$ para $u \in \partial Q$

$\left(\Gamma_{2}\right) g(u)=s(u) e^{\theta(u) L} u+K(u)$ onde $\theta: E \rightarrow \mathbb{R}$ é Lipschitz, $K: E \rightarrow E$ é compacta e Lipschitz e $s: E \rightarrow[0,1]$ é Lipschitz com $s(E)>s_{0}>0$.

Seja

$$
c=\inf _{g \in \Gamma} \sup _{u \in Q} f(g(u)) .
$$

Utilizando resultados de Teoria do Grau e também de análise funcional, mostra-se em [1] que $c \geq \alpha$.

Mostremos agora que $c$ é de fato valor crítico para $f$. Suponhamos por contradição que não seja. Como $c \geq \alpha>\sup _{\partial Q} f$, tomando $\bar{\epsilon}=\frac{c-\sup _{\partial Q} f}{2}$ o Lema da Deformação (ver [7] ou [14]) nos garante a existência de um $\epsilon<\bar{\epsilon}$ e uma deformação $\eta:[0,1] \times E \rightarrow E$ tal que $\eta\left(1, f^{c+\epsilon}\right) \subset f^{c-\epsilon}$ e $\eta(t, u)=u \quad \forall t \in[0,1]$ se $f(u) \notin[c-\epsilon, c+\epsilon]$. Escolha agora uma $g \in \Gamma \operatorname{com} f(g(u))<c+\epsilon \forall u \in Q$. Então se $u \in \partial Q, g(u)=u$ e então $f(g(u))<\sup _{\partial Q} f<c-\frac{\left(c-\sup _{\partial Q} f\right)}{2}=c-\bar{\epsilon}<c-\epsilon, \operatorname{assim} \eta(1, g(u))=g(u)=u$. Logo $\eta(1, g(\cdot))$ satisfaz $(\Gamma 1)$. Por [7], Proposição A.18, a deformação $\eta$ satisfaz também (Г2). Portanto $\eta(1, g(\cdot)) \in \Gamma$. Dessa forma, para todo $u \in Q$

$$
c \leq \sup _{u \in Q} f(\eta(1, g(u)))<c-\epsilon
$$

já que $\eta\left(1, f^{c+\epsilon}\right) \subset f^{c-\epsilon}$, o que nos leva a uma contradição.

Portanto $c$ é valor crítico com $\alpha \leq c$ e $c<\beta$ já que $f(u) \leq \beta \forall u \in Q$.

A estimativa para o índice de Morse relativo segue pela Observação (1.3.6).

Os resultados a seguir serão utilizados na demonstração do Teorema (1.3.3).

Seja $u_{0}$ um ponto crítico não degenerado de $f$ onde $f\left(u_{0}\right)=c$. Suponha que $E=V^{-} \oplus V^{+}$ onde $V^{+}\left(V^{-}\right)$é o autoespaço positivo (negativo) de $f^{\prime \prime}\left(u_{0}\right)$. Seja $P_{+}\left(P_{-}\right)$a projeção de E em $V^{+}\left(V_{-}\right)$. Se $u \in E$, denotemos $u_{+}=P_{+}(u)$ e similarmente para $u_{-}$. 
Seja também $B_{-}=B\left(0, r_{1}\right) \cap V_{-}$e $B_{+}=B\left(0, r_{2}\right) \cap V_{+}$. Tomemos $r_{1}, r_{2}>0$ pequeno o suficiente para que, pelo Lema de Morse exista um $C^{1}$ isomorfismo $\Psi: 2 B_{-} \oplus B_{+} \rightarrow U$ onde $\Psi(0)=u_{0}$ e $U=\Psi\left(2 B_{-} \oplus 2 B_{+}\right)$e

$$
f\left(\Psi\left(z_{+}+z_{-}\right)\right)=c+\left\|z_{+}\right\|^{2}-\left\|z_{-}\right\|^{2} .
$$

Definamos $\Phi: E \rightarrow E$ para $r_{1}, r_{2}>0$ tal que $r_{2}^{2}-4 r_{1}^{2}>0$, da seguinte forma:

$$
\Phi(u)= \begin{cases}u & \text { se } u \notin U \\ \Psi\left(\varphi\left(\frac{\left\|\Psi^{-1}(u)_{-}\right\|}{r_{1}}-1\right) \Psi^{-1}(u)_{+}+\Psi^{-1}(u)_{-}\right) & \text {se } u \in U\end{cases}
$$

onde $\varphi: \mathbb{R} \rightarrow[0,1]$ é Lipschitz e tal que $\varphi(s)=\left\{\begin{array}{lll}0 & \text { se } & s \leq 0 \\ 1 & \text { se } & s \geq 1\end{array}\right.$

Lema 1.3.8. Seja $g \in \Gamma, 0<\alpha_{1}<r_{2}^{2}-4 r_{1}^{2}, c=f\left(u_{0}\right)$ e $U_{1}=\Psi\left(B_{-} \oplus B_{+}\right)$, então:

(i) Se $u \in U \backslash \operatorname{int}\left(U_{1}\right)$, então $f(\Phi(u)) \leq f(u)$;

(ii) Se $u \in \partial U_{1}$ e $f(u) \leq c+\alpha_{1}$, então $\Phi(u)=\Psi\left(\left(\Psi^{-1}(u)\right)_{-}\right)$e $\Phi(u) \in \Psi\left(\partial B_{-}\right)$;

(iii) $\Phi\left(E \backslash \operatorname{int}\left(U_{1}\right)\right) \subset E \backslash \operatorname{int}\left(U_{1}\right)$;

(iv) Suponha que $f(g(Q))<c+\alpha_{1}$ e $f(\partial Q) \leq \alpha<c$. Suponha ainda que $A \neq \emptyset$ onde $A=g^{-1}\left(\operatorname{int}\left(U_{1}\right)\right) \cap Q$. Então $\Phi(g(\partial A)) \subset \Psi\left(\partial B_{-}\left(0, r_{1}\right)\right)$

Demonstração. (i) Se $u \in U \backslash \operatorname{int}\left(U_{1}\right)$, então $\frac{\left\|\Psi^{-1}(u)_{-}\right\|}{r_{1}}-1 \geq 0$, dessa forma $\left\|\varphi\left(\frac{\left\|\Psi^{-1}(u)_{-}\right\|}{r_{1}}-1\right) \Psi^{-1}(u)_{+}\right\| \leq \Psi^{-1}(u)_{+}$. Logo por (1.1), $f(\Phi(u)) \leq f(u)$.

(ii) Como $u \in \partial U_{1}$, então $\Psi^{-1}(u) \in\left(\partial B_{-} \oplus B_{+}\right) \cup\left(B_{-} \oplus \partial B_{+}\right)$. Note que não pode ocorrer de $\Psi^{-1}(u) \in B_{-} \oplus \partial B_{+}$, pois se ocorresse, teríamos

$$
f(u)=f\left(\Psi\left(\Psi^{-1}(u)\right)\right)=c+\left\|\Psi^{-1}(u)_{+}\right\|^{2}-\left\|\Psi^{-1}(u)_{-}\right\|^{2}>c+r_{2}^{2}-4 r_{1}^{2}>c+\alpha_{1}
$$

o que contradiz a hipótese. Logo $\Psi^{-1}(u) \in \partial B_{-} \oplus B_{+}$, ou seja, $\frac{\left\|\Psi^{-1}(u)_{-}\right\|}{r_{1}}-1=0$ e então $\Phi(u)=\Psi\left(\left\{\Psi^{-1}(u)_{-}\right\}\right) \in \Psi\left(\partial B_{-}\right)$.

(iii) Segue pela definição.

(iv) Seja $u \in \partial A$, então supondo que $g^{-1}\left(\operatorname{int}\left(U_{1}\right)\right) \cap \partial Q=\emptyset$ (podemos considerar $r_{1}$ e $r_{2}$ suficientemente pequenos para que isto ocorra), temos $g(u) \in \partial U_{1}$. Como por hipótese $f(g(Q))<c+\alpha_{1}$ e $\partial A \subset \bar{Q}$, então $f(g(\partial A)) \leq c+\alpha_{1}$. Logo pelo item (ii), temos

$$
\Phi(g(u))=\Psi\left(\left\{\Psi^{-1}(u)\right\}_{-}\right) \in \Psi\left(\partial B_{-}\right) \quad \forall u \in \partial A
$$


Vamos assumir os três lemas seguintes para, posteriormente, apresentar a prova do Teorema 1.3 .3

Lema 1.3.9. Seja $X$ um espaço métrico e $Y \subset X$. Se $f: Y \rightarrow \mathbb{R}$ é uma função Lipschitz com constante $\alpha>0$, então $f$ pode ser estendida a uma função de $X$ em $\mathbb{R}$ com a mesma constante de Lipschitz $\alpha$.

Lema 1.3.10. Seja $H$ espaço de Hilbert e $f: H \rightarrow \mathbb{R}$ uniformemente contínua, então $f$ pode ser aproximada uniformemente por funçôes $C^{1}$.

Lema 1.3.11. Sejam $E$ e $F$ dois espaços de Banach separáveis, $h$ uma aplicação $C^{1}$ de Fredholm de indice 0 de $E$ em $F$. Se h não for sobrejetora, então $h$ não é localmente sobrejetora.

Prova do Teorema(1.3.3). Dado $c$ obtido na prova do Teorema (1.3.2), como $f$ satisfaz $(P S)_{c}$, então $K_{c}=\left\{u \in H ; f(u)=c\right.$ e $\left.f^{\prime}(u)=0\right\}$ é compacto. Como por hipótese todos os pontos críticos no nível $c$ são não-degenerados, então $K_{c}$ é formado por uma quantidade finita de pontos.

Suponhamos inicialmente que $K_{c}=\left\{u_{0}\right\}$, os demais casos seguirão por indução. Seja $W_{1}=W^{-} \oplus\{\lambda e ; \lambda \in \mathbb{R}\}$ e suponhamos por absurdo que $\operatorname{dim}_{W_{1}} V^{-}>0$, ou seja, que $0<\operatorname{dim}_{W_{1}} V^{-}=\operatorname{dim}_{W^{-}} V^{-}+\operatorname{dim}_{W_{1}} W^{-}=\operatorname{dim}_{W^{-}} V^{-}-1$, o que é equivalente a supor que $m_{W^{-}, W^{+}}\left(u_{0}, f\right)>1$. Para chegarmos à contradição que provará o teorema, é suficiente encontrarmos um aberto $\tilde{U}$ com $u_{0} \in \tilde{U}$ tal que, para todo $\epsilon$ suficientemente pequeno (menor que um determinado $\bar{\epsilon}$ ), existe $\gamma \in \Gamma$ satisfazendo

$$
\sup _{u \in Q} f(\gamma(u))<c+\epsilon \quad \text { e } \quad \gamma(Q) \cap \tilde{U}=\emptyset .
$$

De fato, dado $\bar{\epsilon}>0$ e $\tilde{U}$ vizinhança de $K_{c}$, existe $0<\epsilon<\bar{\epsilon}$ e uma deformação $\eta:[0,1] \times E \rightarrow E$ contínua tal que:

(i) $\eta(0, u)=u \quad \forall u \in E$,

(ii) $\eta(t, u)=u \quad \forall t \in[0,1]$ e $\forall u$ tal que $f(u) \notin[c-\epsilon, c+\epsilon]$,

(iii) $\eta\left(1, f^{c+\epsilon} \backslash \tilde{U}\right) \subset f^{c-\epsilon}$.

Como $c \geq \alpha \operatorname{esup}_{\partial Q} f<\alpha$, se o $\bar{\epsilon}$ for tomado de forma que $0<\bar{\epsilon}<c-\sup _{\partial Q} f$, então teremos

$$
\max _{u \in \partial Q} f(u)<c-\bar{\epsilon}<c-\epsilon .
$$

Então se $g \in \Gamma$ satisfaz (1.2), defina $g_{1}(u)=\eta(1, g(u))$. Assim, se $u \in \partial Q, g_{1}(u)=$ $\eta(1, g(u))=\eta(1, u)=u$ já que $f(u)<c-\epsilon$. Logo $g_{1} \in \Gamma$ pois $\left(\Gamma_{2}\right)$ segue de [7], Proposição A.18. Note ainda que, por (1.2), $g(Q) \subset f^{c+\epsilon} \backslash \tilde{U}$ e então $g_{1}(Q)=\eta(1, g(Q)) \subset f^{c-\epsilon}$, ou seja, $\left.\sup _{u \in Q} f\left(g_{1}(u)\right)\right)<c-\epsilon<c$, o que contradiz a definição de $c$. 
O resto da prova se resumirá em construir $\gamma$ e $\tilde{U}$ que satisfaçam (1.2).

Seja $\gamma \in \Gamma$ tais que $\sup _{u \in Q} f(g(u))<c+\epsilon \operatorname{com} \epsilon<\alpha_{1}$ e $\alpha_{1}$ é como no Lema 1.3.8. Sendo $A=g^{-1}\left(\operatorname{int}\left(U_{1}\right)\right) \cap Q$, podemos assumir que $A \neq \emptyset$, pois caso contrário $g(Q) \cap U_{1}=\emptyset$ e teríamos satisfeito (1.2), ou seja, nada mais teríamos a provar. Assim como na prova de (iv) do Lema 1.3.8, podemos supor que $g^{-1}\left(\operatorname{int}\left(U_{1}\right)\right) \cap \partial Q=\emptyset$.

Pelo Lema 1.3.8, $\Phi(g(\partial A)) \subset \Psi\left(\partial B_{-}\left(0, r_{1}\right)\right)$. Por outro lado, por [10], o $C^{1}$ isomorfismo $\Psi$ é tal que:

$$
\Psi=(I-K)\left|f^{\prime \prime}\left(u_{0}\right)\right|^{-\frac{1}{2}} \quad \Psi^{-1}=\left|f^{\prime \prime}\left(u_{0}\right)\right|^{\frac{1}{2}}\left(I+K_{1}\right),
$$

onde $K$ e $K_{1}$ são compactos.

Queremos que $\left.\left(\Psi^{-1} \circ g\right)\right|_{\partial A}$ tenha uma extensão suave ao fecho de $A$. Para isso usaremos os Lemas 1.3.9 e 1.3.10. Seja $u \in \partial A$, temos $\Psi^{-1}(g(u))=\left|f^{\prime \prime}\left(u_{0}\right)\right|^{\frac{-1}{2}}\left(I+K_{1}\right) g(u)=$ $\left|f^{\prime \prime}\left(u_{0}\right)\right|^{\frac{1}{2}} s(u) \exp \{\theta(u) L\} u+K(u)$.

Para todo $\epsilon>0$ pequeno o suficiente, existe uma aplicação compacta $K_{\epsilon}$, cuja dimensão da imagem é finita, definida em $\partial A$, tal que $\left\|K_{\epsilon}(u)-K(u)\right\|<\epsilon$. Usando o Lema 1.3.9, existe uma extensão diferenciável compacta $K_{4}$ de $K_{\epsilon}$. Da mesma forma, $\left.\theta\right|_{\partial A}$ pode ser estendida a uma função $\theta_{1}, C^{1}$ definida no fecho de $A$. Dessa forma, a extensão ao fecho de $A$ de $\left(\Psi^{-1} \circ g\right) \mid \partial A$ é $\Psi_{1}(u)=\left|f^{\prime \prime}\left(u_{0}\right)\right|^{\frac{1}{2}} s(u) \exp \left\{\theta_{1}(u) L\right\} u+K_{4}(u)$.

Por conta de

$$
\exp \left\{\theta_{1}(u) L\right\}-\exp \left\{\theta_{1}(u)\left[L+h^{\prime \prime}\left(u_{0}\right)\right]\right\}
$$

ser compacta, temos $\Psi_{1}(u)=\left|f^{\prime \prime}\left(u_{0}\right)\right|^{\frac{1}{2}} s(u) \exp \left\{\theta_{1}(u)\left[L+h^{\prime \prime}\left(u_{0}\right)\right]\right\} u+K_{5}$.

Como $W_{1}$ e $V^{-}$são comensuráveis, então $P_{\left(V^{-}\right)^{\perp}} \circ P_{W_{1}}$ é compacto, ou seja, $P_{\left(V^{-}\right)^{\perp}} \circ$ $\left.P_{W_{1}}\right|_{\bar{A}}=P_{\left(V^{-}\right)^{\perp}}$ é compacto e então $u=P_{V^{-}} u+P_{V^{+}} u$ e isso implica que

$$
u=P_{V^{-}} u+K_{A} u
$$

onde $K_{A}$ é compacto. Assim

$$
P_{-}\left(\Psi_{1}\right)(u)=s(u)\left|f^{\prime \prime}\left(u_{0}\right)\right|^{\frac{1}{2}} \exp \left\{\theta_{1}(u)\left[L+h^{\prime \prime}\left(u_{0}\right)\right]\right\} u+K_{6}(u) .
$$

Usando novamente (1.3) vemos que $\Psi_{2}=P_{-}\left(\Psi_{1}\right)$ é uma aplicação de Fredholm de índice $0\left(\left.\Psi_{2}\right|_{\bar{A}}: \bar{A} \rightarrow V^{-}\right)$. Como $\operatorname{dim}_{W_{1}} V^{-}>0$ então $\Psi_{2}$ não pode ser sobrejetora e pelo Lema 1.3.11, $\Psi_{2}$ não é localmente sobrejetora. Isto implica na existência de um $\delta \in\left(\frac{1}{2} r_{1}, \frac{3}{4} r_{1}\right)$ e $z \in B_{\delta}(0) \cap V^{-}$tal que $z \notin \operatorname{Im}\left(\Psi_{2}\right)$. Podemos agora projetar a porção de $\Psi_{2}(\bar{A})$ que intercepta $B_{\delta}(0)$ em $\partial B_{\delta}(0)$ através de $z$. Assim vemos que $\Psi_{2}$ aplica $\bar{A}$ em $V^{-} \backslash B_{\delta}(0)$.

Definamos agora a aplicação $\Psi_{3}$ por $\Psi_{3}(u)=\frac{\delta}{\left\|\Psi_{2}(u)\right\|} \Psi_{2}(u)$, ou seja

$$
\Psi_{3}(u)=\frac{\delta}{\left\|\Psi_{2}(u)\right\|}\left|f^{\prime \prime}\left(u_{0}\right)\right|^{\frac{1}{2}} s(u) \exp \left\{\theta_{1}(u) L\right\} u+K_{6}(u)
$$

Note que $\Psi_{3}$ é a normalização de $\Psi_{2}$ com relação a fronteira da bola $B_{\delta}(0)$. Pela definição de $\Psi$, temos $\Psi \circ \Psi_{3}(u)=s_{1}(u) \exp \left\{\theta_{1}(u) L\right\} u+K_{7}(u)$ onde $s_{1}(u)=\frac{\delta}{\left\|\Psi_{2}(u)\right\|} s(u) \in\left(s_{0}, 1\right), \operatorname{com}$ $s_{0}>0$ e $\Psi \circ \Psi_{3}(\bar{A}) \subset U \backslash \Psi\left(B_{\delta}(0)\right)$. 
Defina então

$$
\gamma(u)=\left\{\begin{array}{lll}
\Phi(g(u)) & \text { se } & u \notin A \\
\Psi \circ \Psi_{3}(u) & \text { se } & u \in \bar{A}
\end{array} .\right.
$$

Observe que $\Psi \circ \Psi_{3}$ pode diferir de $\Phi \circ g$ em alguns pontos de $\partial A$, mas como $\Psi \circ \Psi_{3}(\partial A)$ está fora de $\Psi\left(B_{\delta}(0)\right)$, podemos pensar que $\Phi \circ g$ e $\Psi \circ \Psi_{3}$ coincidem em $\partial A$.

Afirmamos então que a condição (1.2) vale para $\gamma$ e $\tilde{U}=\Psi\left(B_{\delta}(0)\right)$. Com efeito:

Dado $u \in Q$

- Se $u \notin A$ então como $u \in Q$, temos $g(u) \notin i n t\left(U_{1}\right)$. Pelo Lema 1.3.8 item (iii), $\Phi(g(u)) \in H \backslash \operatorname{int}\left(U_{1}\right)$. Logo, se $u \notin A, \gamma(u) \notin \Psi\left(B_{\delta}(0)\right)$;

- Se $u \in \bar{A}$, pela construção de $\Psi_{3}$, temos $\gamma(u)=\Psi \circ \Psi_{3}(u) \in U \backslash \Psi\left(B_{\delta}(0)\right)$ e então $\gamma(Q) \cap \Psi\left(B_{\delta}(0)\right)=\emptyset$.

Queremos mostrar agora que $\sup _{u \in Q} f(\gamma(u))<c+\epsilon$. Seja $u \in Q$ e suponha inicialmente que $u \notin A$, então $g(u) \notin \operatorname{int}\left(U_{1}\right)$. Temos então duas possibilidades:

- Se $g(u) \in U \backslash U_{1}$, temos pelo Lema 1.3.8 item $(i)$ que $f(\gamma(u))=f(\Phi(g(u))) \leq f(g(u))<$ $c+\epsilon$;

- Se $g(u) \notin U$ então pela definição de $\Phi, \gamma(u)=g(u)$ e novamente $f(\gamma(u))=f(g(u))<$ $c+\epsilon$.

Suponha agora que $u \in A$, então $f(\gamma(u))=f\left(\Psi \circ \Psi_{3}(u)\right)=c-\left\|P_{-}\left(\Psi_{3}(u)\right)\right\|^{2}<c<c+\epsilon$, pois $P_{+}\left(\Psi_{3}(u)\right)=0$.

Isto encerra portanto a prova. 


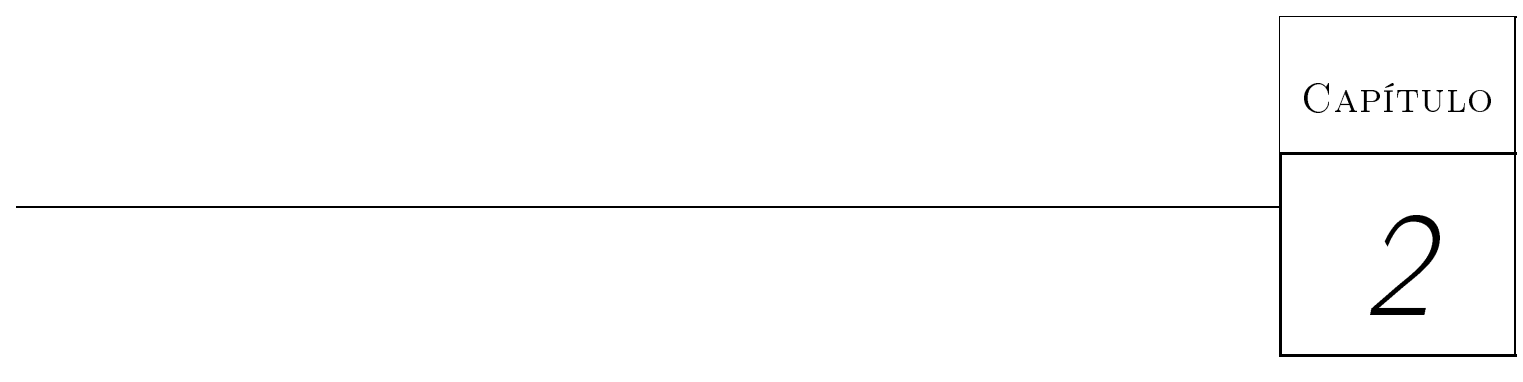

\section{Existência e concentração de} soluções para um sistema elíptico

\subsection{Introdução}

Nesse capítulo, vamos estudar a existência e algumas características interessantes das soluções do seguinte sistema elíptico com condição de Neumann na fronteira:

$$
\left\{\begin{array}{rlll}
-\epsilon^{2} \Delta u+u & =g(v) & \text { em } \Omega \\
-\epsilon^{2} \Delta v+v & =f(u) & \text { em } \Omega \\
\frac{\partial u}{\partial n}=\frac{\partial v}{\partial n} & =0 & \text { sobre } \partial \Omega
\end{array}\right.
$$

onde $\epsilon>0$ é pequeno, $\Omega \subset \mathbb{R}^{N}, N \geq 3$ é um domínio $C^{2}$ limitado e $n(x)$ é o vetor normal a $\partial \Omega$ em $x$.

Verificaremos que, para valores de $\epsilon$ suficientemente pequenos, as soluções $u_{\epsilon}, v_{\epsilon}$ apresentam a interessante característica de atingir o seu máximo em um único e comum ponto da fronteira de $\Omega$. Para isso, será muito importante aplicar toda a teoria estudada no capítulo anterior, sobre tudo as estimativas de Morse para os pontos críticos do funcional energia associado.

Para a verificação de todas as hipóteses e condições geométricas exigidas para a aplicação do Teorema (1.3.2), será necessário considerar algumas hipóteses sobre as não-linearidades $f$ e $g$. 
A técnica utilizada nesse capítulo tanto para a obtenção das soluções como para o seu posterior estudo qualitativo, seguiu o trabalho [8] de Ramos e Yang.

\subsection{Estimativas a priori de soluções}

Consideremos o sistema (2.1), onde as não linearidades $f$ e $g$ satisfazem as seguintes hipóteses:

(H) $f, g \in C^{1}(\mathbb{R}), f(0)=g(0)=f^{\prime}(0)=g^{\prime}(0)=0$ e existem $l_{1}, l_{2}>0$ e $p, q>2$ tais que

$$
\frac{1}{p}+\frac{1}{q}>\frac{N-2}{N}
$$

e

$$
\lim _{|s| \rightarrow \infty} \frac{f^{\prime}(s)}{|s|^{p-2}}=l_{1} \quad \text { e } \quad \lim _{|s| \rightarrow \infty} \frac{g^{\prime}(s)}{|s|^{q-2}}=l_{2} .
$$

Além disso, para algum $\delta>0$ e todo $s \in \mathbb{R}, s \neq 0$,

$$
f(s) s \geq(2+\delta) F(s)>0 \quad \text { e } \quad f^{2}(s) \leq 2 f^{\prime}(s) F(s)
$$

e igualmente para $g$.

Note que no caso em que $f$ é convexa, ou seja, $f^{\prime \prime} \geq 0$, a desigualdade $f^{2}(s) \leq 2 f^{\prime}(s) F(s)$ é satisfeita, pois, sendo $h(s)=2 f^{\prime}(s) F(s)-f^{2}(s)$, temos

$$
h^{\prime}(s)=2 f^{\prime \prime}(s) F(s)+2 f^{\prime}(s) f(s)-2 f(s) f^{\prime}(s)=2 f^{\prime \prime}(s) F(s) \geq 0 .
$$

Pois $f^{\prime \prime} \geq 0$ e $F(s) \geq 0$, já que $f(0)=f^{\prime}(0)=0$ e $f^{\prime \prime} \geq 0$ não permite que $f$ decresça. Logo, como $h(0)=0$ e $h^{\prime} \geq 0$, temos $h(s) \geq 0$.

Como modelo de não linearidades que satisfazem tais hipóteses, poderíamos considerar

$$
f(s)=|s|^{p-1} \quad \text { e } \quad g(s)=|s|^{q-1}
$$

Devido a (2.1) se tratar de um sistema Hamiltoniano, o respectivo funcional energia é dado por:

$$
I(u, v)=\int_{\Omega}\left(\epsilon^{2}\langle\nabla u, \nabla v\rangle+u v-F(u)-G(v)\right) d x
$$

onde $F(\xi)=\int_{0}^{\xi} f(t) d t$ e $G(\xi)=\int_{0}^{\xi} g(t) d t$. Observando a condição (2.2) sobre $p$ e $q$, pode ocorrer que, por exemplo, $p<2^{*}<q$, onde $2^{*}=\frac{2 N}{N-2}$ é o expoente crítico de Sobolev e, dessa forma, $I$ poderia não estar bem definido em $H^{1}(\Omega) \times H^{1}(\Omega)$. Como exemplo onde tal situação poderia ocorrer, tomemos $f$ e $g$ como em $(2.5)$, então $G(v)=\frac{|v|^{q}}{q}$, porém $H^{1}(\Omega)$ não está imerso em $L^{q}(\Omega)$ para $q>2^{*}$. Dessa forma, $v$ pode não ser q-integrável, ou seja, $\int_{\Omega} G(v) d x=\infty$. Para contornar este problema vamos obter uma estimativa a priori para a norma $L^{\infty}$ das soluções, que nos permitirá supor, sem perda de generalidade, que $2<p=q<2^{*}$. Suponhamos então que $2<p \leq q$ e $p<2^{*}$ e, para uma dada sequência $\left(a_{j}\right) \subset \mathbb{R}, a_{j} \rightarrow+\infty$, seja, para cada $j \in \mathbb{N}$ 


$$
g_{j}(s)=\left\{\begin{array}{l}
A_{j}|s|^{p-2} s+B_{j} \quad s \geq a_{j} \\
g(s) \\
\tilde{A}_{j}|s|^{p-2} s+\tilde{B}_{j} s \mid \leq a_{j}
\end{array}\right.
$$

onde os coeficientes devem ser tomados de maneira que $g_{j} \in C^{1}(\mathbb{R})$.

Como $a_{j} \rightarrow+\infty$ quando $j \rightarrow \infty$, então $\lim _{a_{j} \rightarrow+\infty} \frac{g^{\prime}\left(a_{j}\right)}{\left|a_{j}\right|^{q-2}}=l_{2}$. Então, pela continuidade de $g_{j}^{\prime}, \lim _{j \rightarrow \infty}(p-1) A_{j} a_{j}^{p-q}=l_{2}$. Portanto é suficiente definir $A_{j}=a_{j}^{q-p}\left(\frac{l_{2}}{p-1}+o(1)\right)$.

Da mesma forma, segue que $\tilde{A}_{j}=-A_{j}$. Para $B_{j}$ e $\tilde{B}_{j}$, note que

$$
\lim _{a_{j} \rightarrow \infty} \frac{g_{j}\left(a_{j}\right)}{\left|a_{j}\right|^{q-1}}(q-1)=\lim _{a_{j} \rightarrow \infty} \frac{g_{j}^{\prime}\left(a_{j}\right)}{\left|a_{j}\right|^{q-2}}=l_{2} .
$$

Pela continuidade de $g_{j}$, é suficiente considerar

$$
B_{j}=\left(\frac{l_{2}(p-q)}{(p-1)(q-1)}+o(1)\right) a_{j}^{q-1}=-\tilde{B}_{j} .
$$

Consideremos agora o problema modificado:

$$
\left\{\begin{array}{rlll}
-\epsilon^{2} \Delta u+u & =g_{j}(v) & \text { em } \Omega \\
-\epsilon^{2} \Delta v+v & =f(u) & \text { em } \Omega \\
\frac{\partial u}{\partial n}=\frac{\partial v}{\partial n} & =0 & \text { sobre } \partial \Omega
\end{array}\right.
$$

O correspondente funcional energia, que continuaremos a denotar por $I$, é dado por:

$$
I(u, v)=\int_{\Omega}\left(\epsilon^{2}\langle\nabla u, \nabla v\rangle+u v-F(u)-G_{j}(v)\right) d x
$$

onde $G_{j}(\xi)=\int_{0}^{\xi} g_{j}(t) d t$. Note que agora $I$ está bem definido sobre $H^{1}(\Omega) \times H^{1}(\Omega)$, pois, dado $u, v \in H^{1}(\Omega)$ :

$$
\begin{aligned}
\int_{\Omega} G_{j}(v) d x & =\int_{\Omega_{a_{j}}} G_{j}(v) d x+\int_{\Omega \backslash \Omega_{a_{j}}} G_{j}(v) d x \\
& =\int_{\Omega_{a_{j}}} G(v) d x+\int_{\Omega \backslash \Omega_{a_{j}}} \frac{A_{j}}{p}|v|^{p}+B_{j}|v| d x<\infty
\end{aligned}
$$

onde $\Omega_{a_{j}}=\left\{x \in \Omega ;|v(x)| \leq a_{j}\right\}$, já que $H^{1}(\Omega)$ está imerso em $L^{p}(\Omega)$ e $L^{1}(\Omega)$. Temos ainda que $I$ é um funcional $C^{2}$.

Para tratar o problema (2.1) fazendo uso do Capítulo 1, vamos munir o espaço de Hilbert $E=H^{1}(\Omega) \times H^{1}(\Omega)$ com o seguinte produto interno:

$$
\left(\left(u_{1}, v_{1}\right),\left(u_{2}, v_{2}\right)\right)=\int_{\Omega}\left(\epsilon^{2}\left(\left\langle\nabla u_{1}, \nabla u_{2}\right\rangle+\left\langle\nabla v_{1}, \nabla v_{2}\right\rangle\right)+u_{1} u_{2}+v_{1} v_{2}\right) d x
$$


Assim, considerando o operador invertível auto-adjunto $L: E \longrightarrow E$, dado por $L(u, v)=$ $(v, u)$, podemos escrever o funcional $I$ como:

$$
I(u, v)=\frac{1}{2}(L(u, v),(u, v))+h(u, v)
$$

onde $h: H^{1}(\Omega) \times H^{1}(\Omega) \longrightarrow \mathbb{R}$ é dado por $h((u, v))=-\int_{\Omega}\left(F(u)+G_{j}(v)\right) d x$. Note que $h$ é $C^{2}$ e $h^{\prime}$ é compacto. Os pontos críticos de $I$ correspondem a soluções fracas de (2.8), e por um argumento de bootstrap combinado com o resultado de regularidade em [11], tais soluções são clássicas. Temos ainda que a derivada segunda de $I$ é dada por:

$$
I^{\prime \prime}(u, v)(\phi, \psi)(\phi, \psi)=\int_{\Omega}\left(2 \epsilon^{2}\langle\nabla \phi, \nabla \psi\rangle+2 \phi \psi-f^{\prime}(u) \phi^{2}-g_{j}^{\prime}(v) \psi^{2}\right) .
$$

Sejam $E^{+}=\left\{(\phi, \phi) ; \phi \in H^{1}(\Omega)\right\}$ e $E^{-}=\left\{(\phi,-\phi) ; \phi \in H^{1}(\Omega)\right\}$, os autoespaços positivo e negativo, respectivamente, de $L$. Então temos a seguinte fatoração $E=E^{+} \oplus E^{-}$. Denotemos por $Q$, a parte quadrática do funcional I, ou seja:

$$
Q(u, v)=\int_{\Omega}\left(\epsilon^{2}\langle\nabla u, \nabla v\rangle+u v\right) .
$$

Temos que $Q$ é não negativo em $E^{+}$e não positivo em $E^{-}$. Se $(u, v)$ é solução de (2.8), denotamos por $m(u, v)$ o seu índice de Morse relativo, tal como definido no Capítulo 1. Vamos então enunciar o resultado principal dessa seção.

Teorema 2.2.1. Suponha verdadeira a hipótese $(H)$. Para qualquer sequência $\epsilon_{j} \rightarrow 0$ e $a_{j} \rightarrow \infty$, seja $\left(u_{j}, v_{j}\right)$ solução do problema (2.8) com $\epsilon=\epsilon_{j}$. Se existir $k \in \mathbb{N}$ tal que $m\left(u_{j}, v_{j}\right) \leq k \forall j \in \mathbb{N}$, então existe $K>0$ tal que:

$$
\left\|u_{j}\right\|_{L^{\infty}}+\left\|v_{j}\right\|_{L^{\infty}} \leq K \quad \forall j \in \mathbb{N} .
$$

Em particular, $\left(u_{j}, v_{j}\right)$ são soluçôes de (2.1), para $j$ suficientemente grande.

Observação 2.2.2. Para provar o resultado principal desse capítulo, será tomado uma sequência $\epsilon_{j} \rightarrow 0$, a qual corresponderá uma sequência de soluções $\left(u_{\epsilon_{j}}, v_{\epsilon_{j}}\right)$, cujo índice de Morse relativo será uniformemente limitado. Dessa forma o Teorema 2.2.1 nos permitirá supor que, sem perda de generalidade, $p, q$ na hipótese $(H)$ são tais que $2<p=q<2^{*}$. Com efeito, pelo Teorema 2.2.1, existe um $K \in \mathbb{R}$ que limita uniformemente a norma $L^{\infty}$ de $\left(u_{\epsilon_{j}}, v_{\epsilon_{j}}\right)$. Então mesmo que $p$ ou $q$ forem maiores que $2^{*}$, como $a_{j} \rightarrow \infty$, então para valores suficientemente grandes de $j$, conseguimos obter soluções do problema original (2.1).

A prova deste teorema usará, entre outros, o seguinte resultado:

Lema 2.2.3. Consideremos a hipótese $(H)$ e seja $(u, v)$ solução de $(P)_{j}$. Se existir $\lambda>0$ e $k+1$ funções em $H^{1}(\Omega), \phi_{1}, \ldots, \phi_{k+1}$, tendo suportes disjuntos e tais que:

$$
I^{\prime \prime}(u, v)\left(\phi_{i}, \lambda \phi_{i}\right)\left(\phi_{i}, \lambda \phi_{i}\right)<0 \quad \forall i \in\{1, \ldots, k+1\},
$$

então $m(u, v) \geq k+1$. 
Demonstração. Seja $V$ o autoespaço negativo de $I^{\prime \prime}(u, v)$. Por definição $m(u, v)=$ $m_{\left(E^{+}, E^{-}\right)}(u, v)=\operatorname{dim}_{E^{-}} V$, onde a dimensão de $V$ relativa a $E^{-}$foi definida na seção (1.2). Seja $W=\left\{(\lambda \phi,-\phi) ; \phi \in H^{1}(\Omega)\right\}$ e vamos usar a seguinte identidade enunciada na seção $(1.2)$ :

$$
\operatorname{dim}_{E^{-}} V=\operatorname{dim}_{W} V+\operatorname{dim}_{E^{-}} W .
$$

Mostremos que $\operatorname{dim}_{E^{-}} W=0$. De fato, como $W^{\perp}=\left\{(\phi, \lambda \phi) ; \phi \in H^{1}(\Omega)\right\}$, então $\left(E^{-}\right)^{\perp} \cap W=E^{+} \cap W=0$ e $E^{-} \cap W^{\perp}=0$, de maneira que $\operatorname{dim}_{E^{-}} W=0$. Logo $m(u, v)=\operatorname{dim}_{E^{-}} V=\operatorname{dim}_{W} V$.

Por $(H)$ temos $f^{\prime} \geq 0$ e $g^{\prime} \geq 0$, então, para todo $\phi \in H^{1}(\Omega)$,

$$
I^{\prime \prime}(u, v)(\lambda \phi,-\phi)(\lambda \phi,-\phi)=\int_{\Omega}\left(2 \epsilon^{2}\langle\nabla(\lambda \phi), \nabla(-\phi)\rangle-2 \lambda \phi^{2}-f^{\prime}(u) \lambda^{2} \phi^{2}-g_{j}^{\prime}(v) \phi^{2}\right)<0,
$$

o que implica que $W \subset V$. Então segue que $\operatorname{dim}_{W} V=\operatorname{dim}\left(V \cap W^{\perp}\right)-\operatorname{dim}\left(V^{\perp} \cap W\right)$, mas $\operatorname{dim}\left(V^{\perp} \cap W\right)=0$, pois como $W \subset V$, segue que $V^{\perp} \subset W^{\perp}$. Portanto $m(u, v)=$ $\operatorname{dim}\left(V \cap W^{\perp}\right)$.

Considere agora $X=\left\{\left(\phi_{i}, \lambda \phi_{i}\right) ; i=1, \ldots, k+1\right\}$. Por hipótese $X \subset V$ e por definição $X \subset W^{\perp}$. Como $\operatorname{dim} X=k+1$, já que os $k$ elementos de $X$ são linearmente independentes, uma vez que os suportes são disjuntos, e $X \subset V \cap W^{\perp}$, então $m(u, v) \geq k+1$.

Definição 2.2.4. Seja $f_{\infty}, g_{\infty} \in C^{1}(\mathbb{R})$ e $u, v \in C^{2}(\Omega)$ soluções do sistema

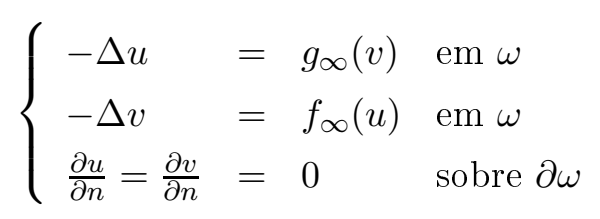

onde $\omega=\left\{x \in \mathbb{R}^{N} ;\left\langle x, y_{0}\right\rangle<d_{0}\right\}$ para algum $y_{0} \neq 0$ e $d_{0} \in(-\infty,+\infty]$.

Dizemos que $(u, v)$ tem índice finito se existir $R_{0}>0$ tal que $\forall \phi \in H^{1}(\Omega) \operatorname{com} \phi=0$ em $B_{R_{0}} \cap \omega$, vale:

$$
2 \int_{\omega}|\nabla \phi|^{2}-\int_{\omega} f_{\infty}^{\prime}(u) \phi^{2}-\int_{\omega} g_{\infty}^{\prime}(v) \phi^{2} \geq 0
$$

No próximo resultado consideraremos $g_{\infty} \in C^{1}$ tal que, existem valores $c_{1}, c_{2}>0,2<$ $p<2^{*}, p \leq q$ e $\frac{1}{p}+\frac{1}{q}>\frac{N-2}{N}$ para os quais valem:

1. $c_{1}|s|^{q} \leq g_{\infty}(s) s \leq c_{2}|s|^{q}$

2. $g_{\infty}(s) s \leq q G_{\infty}(s)$;

3. $(p-1) g_{\infty}(s) s \leq g_{\infty}^{\prime}(s) s^{2}$

$\forall s \in \mathbb{R}$.

A proposição seguinte é um resultado do tipo Liouville que será utilizado na demonstração do Teorema 2.2.1, cujas idéias da prova do item 1, estão presentes no trabalho [9] de Ramos, Terracini e Troestler. 
Proposição 2.2.5. Seja $g_{\infty} \in C^{1}(\mathbb{R})$ e $f_{\infty}(s)=c|s|^{p-2} s$ com $c>0$ e $2<p<2^{*}$ e suponha que $(u, v)$ tenha indice finito e seja limitado em $L^{\infty}(\omega) \times L^{\infty}(\omega)$. Então:

1. Se $g_{\infty}=0$, então $u=0$;

2. Se $g_{\infty}$ satisfaz as propriedades da definição acima, então $u=v=0$.

Demonstração. Como o problema é invariante por translação, podemos sempre supor que $\omega=\mathbb{R}^{N}$ ou que $\omega=\omega_{0}=\left\{x ;\left\langle x, y_{0}\right\rangle<0\right\}$. De fato:

- Se $d_{0}=\infty$, então $\omega=\left\{x ;\left\langle x, y_{0}\right\rangle<\infty\right\}=\mathbb{R}^{N}$.

- Se $d_{0} \in \mathbb{R}$, então sendo $z_{0} \in \mathbb{R}^{N}$ tal que $\left\langle z_{0}, y_{0}\right\rangle=d_{0}$, note que $\omega-z_{0}=\left\{x-z_{0} ;\left\langle x, y_{0}\right\rangle<\right.$ $\left.d_{0}=\left\langle z_{0}, y_{0}\right\rangle\right\}=\left\{x-z_{0} ;\left\langle x-z_{0}, y_{0}\right\rangle<0\right\}=\omega_{0}$.

Portanto não há perda de generalidade em supor que $\omega=\mathbb{R}^{N}$ ou que $\omega=\left\{x ;\left\langle x, y_{0}\right\rangle<0\right\}$ (que é o semi-espaço limitado pelo plano normal a $y_{0}$ ).

Suponhamos que $c=1 \mathrm{e}$, para todo $R>0$ suficientemente grande, seja $\varphi \in C_{0}^{\infty}\left(\mathbb{R}^{N}\right)$ com $0 \leq \varphi \leq 1, \varphi=0$ em $B_{R_{0}} \cup \mathbb{R}^{N} \backslash B_{2 R}(0), \varphi=1$ em $B_{R} \backslash B_{2 R_{0}}$ e $\|\nabla \varphi\|_{L^{\infty}}<\frac{C}{R^{\frac{N+1}{2}}}$, onde $C$ não depende de $R$.

1. Suponhamos que $g_{\infty}=0$, então $(u, v)$ satisfaz o seguinte sistema:

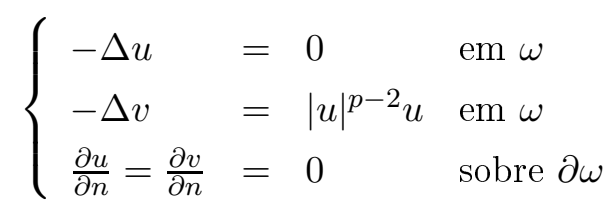

Suponhamos que $c=1$ e, para todo $R>0$ suficientemente grande, seja $\varphi \in C_{0}^{\infty}\left(\mathbb{R}^{N}\right)$ com $0 \leq \varphi \leq 1, \varphi=0$ em $B_{R_{0}} \cup \mathbb{R}^{N} \backslash B_{2 R}(0), \varphi=1$ em $B_{R} \backslash B_{2 R_{0}}$ e $|\nabla \varphi|<\frac{C}{R}$ em $\mathbb{R}^{N} \backslash B_{R}$, onde $C$ não depende de $R$. Pela escolha de $\varphi$, temos $u \varphi \in H^{1}(\omega)$ e $u \varphi=0$ em $\omega \cap B_{R_{0}}$. Multiplicando a primeira equação do sistema acima por $u \varphi^{2} \in H^{1}(\omega)$ e integrando por partes, temos $\int_{\omega}\left(2 u \varphi\langle\nabla u, \nabla \varphi\rangle+\varphi^{2}|\nabla u|^{2}\right)=0$. Usando então o fato de $(u, v)$ ter índice finito, teremos:

$$
2 \int_{\omega}|\nabla(u \varphi)|^{2}-\int_{\omega}(p-1)|u|^{p-2}|u \varphi|^{2} \geq 0 .
$$

Dessa forma

$$
\begin{aligned}
(p-1) \int_{\omega}|u|^{p} \varphi^{2} & \leq 2 \int_{\omega}|\nabla(u \varphi)|^{2} \\
& =2 \int_{\omega}|\varphi \nabla u+u \nabla \varphi|^{2} \\
& =2 \int_{\omega}\left(u^{2}|\nabla \varphi|^{2}+\varphi^{2}|\nabla u|^{2}+2 u \varphi\langle\nabla u, \nabla \varphi\rangle\right) \\
& =2 \int_{\omega} u^{2}|\nabla \varphi|^{2} .
\end{aligned}
$$


Usando agora o fato de $\nabla \varphi=0$ onde $\varphi$ é constante e a limitação de $u$, segue que

$$
\begin{aligned}
(p-1) \int_{B_{R}}|u|^{p} \leq & 2 \int_{B_{2 R}} u^{2}|\nabla \varphi|^{2} \\
= & 2 \int_{B_{R_{0}}} u^{2}|\nabla \varphi|^{2}+2 \int_{B_{2 R_{0} \backslash B_{R_{0}}} u^{2}|\nabla \varphi|^{2}+} u^{2}|\nabla \varphi|^{2}+2 \int_{B_{2 R} \backslash B_{R}} u^{2}|\nabla \varphi|^{2} \\
& 2 \int_{B_{R} \backslash B_{2 R_{0}}} u^{\leq} \\
& C\left(1+\frac{1}{R^{2}} \int_{B_{2 R}} u^{2}\right),
\end{aligned}
$$

ou seja

$$
(p-1) \int_{B_{R}}|u|^{p} \leq C\left(1+R^{-2} \int_{B_{2 R}} u^{2}\right) .
$$

Mostremos que $\int_{B_{R}}|u|^{p}$ é limitado independentemente de $R$.

Suponhamos que não, então para $R$ suficientemente grande, $\int_{B_{R}}|u|^{p}>C$, de maneira que por (2.12)

$$
(p-1) \int_{B_{R}}|u|^{p} \leq \int_{B_{R}}|u|^{p}+C R^{-2} \int_{B_{2 R}} u^{2},
$$

e então

$$
\int_{B_{R}}|u|^{p} \leq C R^{-2} \int_{B_{2 R}} u^{2}
$$

Utilizando a desigualdade de Hölder com $\frac{p}{2}$ e $\frac{p}{p-2}$ na integral da direita de (2.13), teremos

$$
C R^{-2} \int_{B_{2 R}} u^{2} \leq C R^{-2}\left(\int_{B_{2 R}}\left(u^{2}\right)^{\frac{p}{2}}\right)^{\frac{2}{p}} R^{\frac{N p}{p-2}}=C\left(\int_{B_{2 R}}|u|^{p}\right)^{\frac{2}{p}} R^{N \frac{p-2}{p}-2} .
$$

Note agora que, como $p<2^{*}=\frac{2 N}{N-2}$, então:

$$
\frac{N(p-2)}{p}-2=\frac{p(N-2)-2 N}{p}<0
$$

e definimos por $\alpha=2-\frac{N(p-2)}{p}>0$. Então

$$
R^{-2} \int_{B_{2 R}} u^{2} \leq C R^{-\alpha}\left(\int_{B_{2 R}}|u|^{p}\right)^{\frac{2}{p}} .
$$

Aplicando então (2.14) em (2.13), teremos

$$
\int_{B_{R}}|u|^{p} \leq C R^{-\alpha}\left(\int_{B_{2 R}}|u|^{p}\right)^{\frac{2}{p}}
$$

Procedendo de maneira recursiva sobre a desigualdade (2.15), onde reaplicamos a desigualdade às bolas de raio $2^{j} R$, teremos

$$
\begin{aligned}
\int_{B_{R}}|u|^{p} & \leq C R^{-\alpha\left(1+\frac{2}{p}+\ldots+\left(\frac{2}{p}\right)^{k-1}\right)}\left(\int_{B_{2^{k} R}}|u|^{p}\right)^{\left(\frac{2}{p}\right)^{k}} \\
& \leq C R^{-\alpha\left(1+\ldots+\left(\frac{2}{p}\right)^{k-1}\right)+N\left(\frac{2}{p}\right)^{k}}
\end{aligned}
$$


e como $p>2$, para $k$ suficientemente grande, $-\alpha\left(1+\ldots+\left(\frac{2}{p}\right)^{k-1}\right)+N\left(\frac{2}{p}\right)^{k}<0$. Fazendo então $R \rightarrow+\infty$, segue que

$$
\int_{B_{R}}|u|^{p}<C
$$

onde $C$ não depende de $R$. Portanto $u \in L^{p}(\omega)$.

Este fato implica então que

$$
\int_{B_{R}} u^{2}=o\left(R^{2}\right)
$$

De fato, usando a desigualdade de Hölder novamente com $\frac{p}{2}$ e $\frac{p}{p-2}$ e também (2.16), segue que

$$
\begin{aligned}
\int_{B_{R}} u^{2} & \leq\left(\int_{B_{R}}\left(u^{2}\right)^{\frac{p}{2}}\right)^{\frac{2}{p}} R^{\frac{N(p-2)}{p}} \\
& \leq C R^{\frac{N(p-2)}{p}},
\end{aligned}
$$

então

$$
R^{-2} \int_{B_{R}} u^{2} \leq C R^{\frac{N(p-2)}{p}-2}=C R^{-\alpha} \rightarrow 0
$$

quando $R \rightarrow+\infty$.

Mostremos agora que $\int_{\omega}|\nabla u|^{2}=0$. Tomemos então $\varphi \in C_{0}^{\infty}\left(\mathbb{R}^{N}\right), 0 \leq \varphi \leq 1, \varphi=0$ em $B_{2 R}^{c}, \varphi=1$ em $B_{R}$ e $\|\nabla \varphi\|_{L^{\infty}}<\frac{C}{R}$, onde $C$ não depende de $R$.

Como visto anteriormente, $\int_{\omega}\left(2 u \varphi\langle\nabla u, \nabla \varphi\rangle+\varphi^{2}|\nabla u|^{2}\right)=0$, de maneira que, utilizando novamente Hölder,

$$
\begin{aligned}
\int_{\omega} \varphi^{2}|\nabla u|^{2} & \leq 2 \int_{\omega}|u||\varphi||\nabla u||\nabla \varphi| \\
& \leq \frac{C}{R} \int_{\omega}|u\|\varphi\| \nabla u| \\
& \leq \frac{C}{R}\left(\int_{\omega} u^{2}\right)^{\frac{1}{2}}\left(\int_{\omega} \varphi^{2}|\nabla u|^{2}\right)^{\frac{1}{2}} \\
& =C\left(R^{-2} \int_{\omega} u^{2}\right)^{\frac{1}{2}}\left(\int_{\omega} \varphi^{2}|\nabla u|^{2}\right)^{\frac{1}{2}}
\end{aligned}
$$

e isto implica que

$$
\left(\int_{\omega} \varphi^{2}|\nabla u|^{2}\right)^{\frac{1}{2}} \leq C\left(R^{-2} \int_{\omega} u^{2}\right)^{\frac{1}{2}} \rightarrow 0
$$

quando $R \rightarrow+\infty$.

Portanto $\lim _{R \rightarrow+\infty} \int_{B_{R}} \varphi^{2}|\nabla u|^{2}=0$ e então $\int_{\omega}|\nabla u|^{2}=0$. Como $\nabla u=0$ em $\omega$ e $u \in L^{p}(\omega)$, então necessariamente $u=0$ em $\omega$. 
2. Seja $\varphi$ como no item anterior. Por (2.11), teremos:

$$
(p-1) \int_{\omega} f_{\infty}(u) u \varphi^{2}+\int_{\omega} g_{\infty}^{\prime}(v) u^{2} \varphi^{2} \leq 2 \int_{\omega}|\nabla(u \varphi)|^{2}
$$

Argumentando como no item anterior, usando o item (3) da página 19 e a desigualdade $2 u v \leq u^{2}+v^{2}$, chegaremos a:

$$
\begin{aligned}
(p-1) \int_{\omega} f_{\infty}(u) u \varphi^{2}+\epsilon_{\omega} g_{\infty}^{\prime}(v) u^{2} \varphi^{2} & \leq \int_{\omega}|\nabla(u \varphi)|^{2} \\
& =2 \int_{\omega}\left(u^{2}|\nabla \varphi|^{2}+2 u \varphi \nabla u \nabla \varphi+\varphi^{2}|\nabla u|^{2}\right) \\
& =C \int_{\omega} u^{2}|\nabla \varphi|^{2}+2 \int_{\omega} g_{\infty}(v) u \varphi^{2} \\
& \leq \int_{\omega} u^{2}|\nabla \varphi|^{2}+\int_{\omega} g_{\infty}^{\prime}(v) u^{2} \varphi^{2}+\int_{\omega} g_{\infty}(v) v \varphi^{2},
\end{aligned}
$$

o que implica que

$$
(p-1) \int_{\omega} f_{\infty}(u) u \varphi^{2} \leq C \int_{\infty} u^{2}|\nabla \varphi|^{2}+\int_{\omega} g_{\infty}(v) v \varphi^{2} .
$$

Logo teremos

$$
(p-1) \int_{\omega} f_{\infty}(u) u^{2} \varphi^{2} \leq C\left(1+\int_{\omega} u^{2}|\nabla \varphi|^{2}\right)+\int_{\omega} g_{\infty}(v) v \varphi^{2} .
$$

Pelo Teorema 5A de [2], para todo $\epsilon>0$,

$$
\int_{\omega} g_{\infty}(v) v \varphi^{2} \leq \frac{1+\epsilon}{1-\epsilon} \int_{\omega} f_{\infty}(u) u \varphi^{2}+o(1) \quad \text { quando } R \rightarrow \infty
$$

Tomando então $\epsilon>0$ tal que $(p-1)-\frac{1+\epsilon}{1-\epsilon}>0$, segue por (2.18) e (2.19) que $u \in L^{p}(\omega)$. Devido a condição (1) da Definição (2.2.4) e de (2.19), temos também que $v \in L^{q}(\omega)$. Pelo Corolário 6B e Lema 6B de [2], temos que:

$$
\int_{\omega}\langle\nabla u, \nabla v\rangle=\int_{\omega} f_{\infty}(u) u=\int_{\omega} g_{\infty}(v) v<\infty
$$

Usando uma identidade do tipo Pohozaev-Rellich (ver [14], pág. 138) em (2.20), teremos que:

$$
\int_{\omega}\langle\nabla u, \nabla v\rangle=\frac{N}{N-2} \int_{\omega}\left(F_{\infty}(u)+G_{\infty}(v)\right)
$$

Segue então pelo item (2) da Definição (2.2.4), por (2.20) e (2.21) que:

$$
\frac{N-2}{N} \int_{\omega}|u|^{p}=\int_{\omega}\left(F_{\infty}(u)+G_{\infty}(v)\right) \geq \frac{1}{p} \int_{\omega}|u|^{p}+\frac{1}{q} \int_{\omega} g_{\infty}(v) v=\left(\frac{1}{p}+\frac{1}{q}\right) \int_{\omega}|u|^{p} .
$$

Como $\frac{1}{p}+\frac{1}{q}>\frac{N-2}{N}$, então $u=v=0$. 
Agora podemos dar a demonstração completa do Teorema 2.2.1.

Demonstração. Mostraremos que, se supormos por contradição, que a menos de subsequência, $\left\|u_{j}\right\|_{L^{\infty}}+\left\|v_{j}\right\|_{L^{\infty}} \rightarrow+\infty$, então será possível encontrar $x_{j} \in \bar{\Omega}, \alpha_{j}, \beta_{j}>0, \lambda_{j} \longrightarrow 0^{+}$, tais que as seguintes funções:

$$
\tilde{u_{j}}(x)=\frac{1}{\alpha_{j}} u_{j}\left(\lambda_{j} \epsilon_{j} x+x_{j}\right) \quad \text { e } \quad \tilde{v}_{j}(x)=\frac{1}{\beta_{j}} v_{j}\left(\lambda_{j} \epsilon_{j} x+x_{j}\right),
$$

que mostraremos que satisfazem o sistema:

$$
\left\{\begin{array}{l}
-\Delta \tilde{u_{j}}+\lambda_{j}^{2} \tilde{u_{j}}=\frac{\lambda_{j}^{2}}{\alpha_{j}} g_{j}\left(\beta_{j} \tilde{v_{j}}\right) \text { em } \Omega_{j}=\frac{1}{\lambda_{j} \epsilon_{j}}\left(\Omega-x_{j}\right) \\
-\Delta \tilde{v_{j}}+\lambda_{j}^{2} \tilde{v_{j}}=\frac{\lambda_{j}^{2}}{\beta_{j}} f\left(\alpha_{j} \tilde{u_{j}}\right) \quad \text { em } \Omega_{j}
\end{array}\right.
$$

são uniformemente limitadas e, a menos de subsequência, convergem em $C_{l o c}^{2}$ para funções $u$ e $v$, respectivamente, que satisfazem o seguinte problema limite:

$$
\left\{\begin{array}{llc}
-\Delta u & =g_{\infty}(v) & \text { em } \omega \\
-\Delta v & =f_{\infty}(u) & \text { em } \omega \\
\frac{\partial u}{\partial n}=\frac{\partial v}{\partial n} & =0 & \text { sobre } \partial \omega
\end{array}\right.
$$

para $f_{\infty}, g_{\infty} \in C^{1}(\omega)$, onde $\omega$ é $\mathbb{R}^{N}$ ou $\left\{x ;\left\langle x, n\left(x_{0}\right)\right\rangle<d_{0}\right\}$ onde $x_{0} \in \partial \Omega \mathrm{e}$

$d_{0}=\lim _{j \rightarrow \infty} \frac{\operatorname{dist}\left(x_{j}, \partial \Omega\right)}{\lambda_{j} \epsilon_{j}}$. Além disso, mostraremos que $(u, v)$ tem índice finito. O argumento conclusivo será a utilização da Proposição 2.2.5 para chegarmos a uma contradição. Isto dependerá das escolhas de $\alpha_{j}, \beta_{j}, \lambda_{j}$ e $x_{j}$ que, por sua vez, dependem do limite de $\frac{a_{j}}{\left\|v_{j}\right\|_{L^{\infty}}}$ quando $j \rightarrow \infty$.

- Mostremos que $\tilde{u}_{j}$ e $\tilde{v}_{j}$ como definidas em (2.22), satisfazem o sistema (2.23). De fato, sendo $y=\lambda_{j} \epsilon_{j} x+x_{j}$, temos:

$$
\Delta \tilde{u}_{j}(x)=\frac{\lambda_{j}^{2} \epsilon_{j}^{2}}{\alpha_{j}} \Delta u_{j}(y)
$$

Como $u_{j}$ satisfaz $-\epsilon_{j}^{2} \Delta u_{j}+u_{j}=g_{j}\left(v_{j}\right)$, então, substituindo e multiplicando por $\frac{\lambda_{j}^{2}}{\alpha_{j}}$, temos:

$$
-\Delta \tilde{u_{j}}+\lambda_{j}^{2} \tilde{u}_{j}=\frac{\lambda_{j}^{2}}{\alpha_{j}} g_{j}\left(\beta_{j} \tilde{v}_{j}\right)
$$

e analogamente para $\tilde{v}_{j}$.

- Mostremos que as funções $u$ e $v$, que são soluções do problema limite (2.24), têm índice finito. Para isto, suponha que não, ou seja, que $\forall R_{0}>0, \exists \varphi \in H^{1}(\omega) \operatorname{com} \varphi=0$ em $B_{R_{0}}$, que podemos supor que tem suporte compacto (já que $C_{0}^{\infty}(\omega)$ é denso em $H^{1}(\omega)$ ), tal que:

$$
E(\varphi)=2 \int_{\omega}|\nabla \varphi|^{2}-f_{\infty}^{\prime}(u) \varphi^{2}-g_{\infty}^{\prime}(v) \varphi^{2}<0
$$


Note que o problema limite (2.24) foi obtido fazendo $j \rightarrow \infty$ no sistema (2.23).

Vamos admitir por um instante as convergências

$$
\lambda_{j}^{2} \frac{\alpha_{j}}{\beta_{j}} f^{\prime}\left(\alpha_{j} \tilde{u}_{j}\right) \rightarrow f_{\infty}^{\prime}(u)
$$

e

$$
\lambda_{j}^{2} \frac{\beta_{j}}{\alpha_{j}} g_{j}^{\prime}\left(\beta_{j} \tilde{v}_{j}\right) \rightarrow g_{\infty}^{\prime}(u)
$$

Consideremos a mudança de variáveis que leva $\Omega_{j}$ em $\Omega$ dada por $x \mapsto \lambda_{j} \epsilon_{j} x+x_{j}$ e seja $\varphi_{j}(x)=\varphi\left(\frac{x-x_{j}}{\lambda_{j} \epsilon_{j}}\right) \in H^{1}(\Omega)$, onde $j$ é grande o suficiente para que $\operatorname{supp}(\varphi) \subset \Omega_{j}$. Por (2.9), pela convergência $C_{l o c}^{2}$ de $\lambda_{j}^{2} \frac{\alpha_{j}}{\beta_{j}} f^{\prime}\left(\alpha_{j} u_{j}\right)$ e o teorema de mudança de variáveis em integral, temos

$$
\frac{1}{\lambda_{j}^{N-2} \epsilon_{j}^{N}} I^{\prime \prime}\left(u_{j}, v_{j}\right)\left(\varphi_{j}, \frac{\beta_{j}}{\alpha_{j}} \varphi_{j}\right)\left(\varphi_{j}, \frac{\beta_{j}}{\alpha_{j} \varphi_{j}}\right) \longrightarrow E(\varphi)<0 .
$$

Seja então $R_{1}>0$ qualquer, existe $\varphi_{1} \in H^{1}(\omega)$ com suporte compacto, $\operatorname{supp}\left(\varphi_{1}\right) \subset$ $\omega \backslash B_{R_{1}}(0)$ e tal que para algum $j_{1} \in \mathbb{N}, I^{\prime \prime}\left(u_{j_{1}}, v_{j_{1}}\right)\left(\varphi_{1, j_{1}}, \frac{\beta_{j_{1}}}{\alpha_{j_{1}}} \varphi_{1, j_{1}}\right)<0$. Tome agora $R_{2}>0$ tal que $\operatorname{supp}\left(\varphi_{1}\right) \subset B_{R_{2}}(0)$. Existirá então $\varphi_{2} \in H^{1}(\omega)$ com suporte compacto, $\operatorname{supp}\left(\varphi_{2}\right) \subset \omega \backslash B_{R_{2}}(0)$ e tal que, para algum $j_{2} \in \mathbb{N}, I^{\prime \prime}\left(u_{j_{2}}, v_{j_{2}}\right)\left(\varphi_{2, j_{2}}, \frac{\beta_{j_{2}}}{\alpha_{j_{2}}} \varphi_{2, j_{2}}\right)<0$. Repetindo o processo $k+1$ vezes, conseguiremos $k+1$ funções, $\varphi_{1, j}, \ldots \varphi_{k+1, j} \in H^{1}(\Omega)$ com suportes disjuntos e tais que $I^{\prime \prime}\left(u_{j}, v_{j}\right)\left(\varphi_{i, j}, \frac{\beta_{j}}{\alpha_{j}} \varphi_{i, j}\right)<0$, onde $i=1, \ldots, k+1$ e $j>\max \left\{j_{1}, \ldots, j_{k+1}\right\}$. Dessa forma, pelo Lema $2.2 .3, m\left(u_{j}, v_{j}\right) \geq k+1$, o que contraria a nossa hipótese. Portanto $(u, v)$ realmente tem índice finito.

Seja

$$
M_{j}=\sup _{x \in \bar{\Omega}}\left\{\max \left\{\left|u_{j}(x)\right|^{\frac{1}{q}},\left|v_{j}(x)\right|^{\frac{1}{p}}\right\}\right\}
$$

que, por hipótese tende a $+\infty$ quando $j \rightarrow \infty$. Seja

$$
l=\lim _{j \rightarrow \infty} \frac{a_{j}}{M_{j}^{p}} \in[0, \infty]
$$

a menos de subsequência.

CASO 1 - Vamos considerar o caso em que $l>0$ (podendo inclusive ser infinito). Seja $\lambda_{j}$ dado por $\lambda_{j}^{2} M_{j}^{p q-p-q}=1$ e $y_{j} \in \bar{\Omega}$ tal que $M_{j}=\max \left\{\left|u_{j}\left(y_{j}\right)\right|^{\frac{1}{q}},\left|v_{j}\left(y_{j}\right)\right|^{\frac{1}{p}}\right\}$ e sejam $\alpha_{j}=M_{j}^{q}$ e $\beta_{j}=M_{j}^{p}$. Seja agora $\tilde{u}_{j}(x)=\frac{1}{\alpha_{j}} u_{j}\left(\lambda_{j} \epsilon_{j} x+y_{j}\right)$ e $\tilde{v}_{j}(x)=\frac{1}{\beta_{j}} v_{j}\left(\lambda_{j} \epsilon_{j} x+y_{j}\right)$. Note que

$$
\left|\tilde{u}_{j}(x)\right|=\frac{1}{M_{j}^{q}}\left|u_{j}\left(\lambda_{j} \epsilon_{j} x+y_{j}\right)\right| \leq \frac{1}{M_{j}^{q}}\left|u_{j}\left(y_{j}\right)\right|=\frac{1}{M_{j}^{q}} M_{j}^{q}=1
$$

e analogamente para $\tilde{v}_{j}$, logo, $\left\|\tilde{u}_{j}\right\|_{L^{\infty}},\left\|\tilde{v}_{j}\right\|_{L^{\infty}} \leq 1$. 
Para mostrar que $\tilde{u}_{j} \rightarrow u$ e $\tilde{v}_{j} \rightarrow v$ em $C_{l o c}^{2}$, note que o operador $L w=-\Delta w+\lambda_{j}^{2} w$ é elíptico, tem coeficientes $C^{\alpha}, \frac{\lambda_{j}^{2}}{\alpha_{j}} g_{j}$ e $\frac{\lambda_{j}^{2}}{\beta_{j}} f\left(\right.$ são $C^{1}(\mathbb{R})$, em particular $\left.C^{\alpha}\right)$. Então utilizando a estimativa de Schauder, vemos que:

$$
\left|\tilde{u}_{j}\right|_{2, \alpha ; \Omega_{j}} \leq C\left(\left\|\tilde{u}_{j}\right\|_{L^{\infty}}+\frac{\lambda_{j}^{2}}{\alpha_{j}}\left|g_{j}\left(\beta_{j} \tilde{v}_{j}\right)\right|_{0, \alpha ; \Omega_{j}}\right)
$$

e

$$
\left|\tilde{v}_{j}\right|_{2, \alpha ; \Omega_{j}} \leq C\left(\left\|\tilde{v}_{j}\right\|_{L^{\infty}}+\frac{\lambda_{j}^{2}}{\beta_{j}}\left|f\left(\alpha_{j} \tilde{u}_{j}\right)\right|_{0, \alpha ; \Omega_{j}}\right),
$$

onde $C$ não depende de $\tilde{u}_{j}$ e $\tilde{v}_{j}$. Como $\left\|\tilde{u}_{j}\right\|_{\infty},\left\|\tilde{v}_{j}\right\|_{\infty} \leq 1$ e devido a (2.3), $\frac{\lambda_{j}^{2}}{\alpha_{j}}\left|g_{j}\left(\beta_{j} \tilde{v}_{j}\right)\right|_{0, \alpha ; \Omega_{j}}$ é uniformemente limitada, então $\left|\tilde{u}_{j}\right|_{2, \alpha ; \Omega_{j}} \leq K$. Isto implica na equicontinuidade e limitação uniforme das sequências de funções e das derivadas até ordem 2. Finalmente, a menos de subsequência, $\tilde{u}_{j} \rightarrow u$ e $\tilde{v}_{j} \rightarrow v$ em $C_{l o c}^{2}$.

Mostremos que $(u, v)$ satisfaz o problema limite (2.24) para funções $f_{\infty}(s)=\frac{l_{1}}{p-1}|s|^{p-2} s$ e $g_{\infty}(s)=\frac{l_{2}}{q-1}|s|^{q-2} s$ se $|s| \leq l$ e $g_{\infty}(s)=\frac{l_{2}}{p-1}|s|^{p-2} s+\frac{l_{2}(p-q)}{(p-1)(q-1)} l^{q-1}$ para $s<l$ e $g$ ímpar.

- $f_{\infty}$ : substituindo os valores de $\alpha_{j}, \beta_{j}$ e $\lambda_{j}$, vemos que:

$$
\frac{\lambda_{j}^{2}}{\beta_{j}} f\left(\alpha_{j} \tilde{u}_{j}\right)=\frac{f\left(M_{j}^{q} \tilde{u}_{j}\right)}{\left(M_{j}^{q}\right)^{p-1}} .
$$

Como $\lim _{|s| \rightarrow \infty} \frac{f(s)}{|s|^{p-1}}=l_{1}(p-1)$, então $\lim _{|s| \rightarrow \infty} \frac{f(C s)}{|s|^{p-1}}=l_{1}(p-1) C^{p-1}$. Então, como $M_{j}^{q} \rightarrow \infty, \lim _{j \rightarrow \infty} \frac{\lambda_{j}^{2}}{\beta_{j}} f\left(\alpha_{j} \tilde{u}_{j}\right)=\lim _{j \rightarrow \infty} \frac{f\left(M_{j}^{q} \tilde{u}_{j}\right)}{\left(M_{j}^{q}\right)^{p-1}}=\frac{l_{1}}{p-1}|u|^{p-1}=f_{\infty}(u)$.

- $g_{\infty}$ : para o caso em que $|v|<l$, procedemos de maneira exatamente igual ao feito para $f$. Por outro lado, para o caso em que $v \geq l$, procedemos como para $f$ utilizando a definição de $g_{j}$ para valores maiores ou iguais a $a_{j}$. Analogamente para o caso em que $v \leq-l$.

Logo, chegaremos a

$$
g_{\infty}(v)=\left\{\begin{aligned}
\frac{l_{2}}{p-1} l^{q-p}|v|^{p-2} v+\frac{l_{2}(p-q)}{(p-1)(q-1)} l^{q-1} & ; \quad v \geq l \\
\frac{l_{2}}{q-1}|v|^{q-2} v & ; \quad|v| \leq l
\end{aligned}\right.
$$

e $g_{\infty}$ é ímpar.

Verifica-se facilmente que $g_{\infty}$ satisfaz as propriedades (1), (2) e (3) da página 19 e podemos então usar o item (2) da Proposição 2.2.5.

Então $u=v=0$, o que contradiz o fato de $\left|\tilde{u}_{j}(0)\right|=1$ ou $\left|\tilde{v}_{j}(0)\right|=1$.

CASO 2 - Suponha agora que $l=0$. Procedendo como no Caso 1, chegaremos ao problema limite:

$$
\left\{\begin{array}{rccc}
-\Delta u & = & 0 & \text { em } \omega \\
-\Delta v & = & \frac{l_{1}}{p-1}|u|^{p-2} u & \text { em } \omega
\end{array}\right.
$$


e pela Proposição 2.2.5 item $1, u=0 . \operatorname{Logo} M_{j}=\left\|v_{j}\right\|_{L^{\infty}}=\left|v_{j}\left(y_{j}\right)\right|$ e $\lim _{j \rightarrow \infty} \frac{a_{j}}{\left\|v_{j}\right\|_{L^{\infty}}}=$ 0 , em particular $\left\|v_{j}\right\|_{L^{\infty}} \rightarrow \infty$. Consideremos agora outros valores para $\lambda_{j}, \alpha_{j}$ e $\beta_{j}$. Seja então $\alpha_{j}=\beta_{j}=\left\|v_{j}\right\|_{L^{\infty}}$ e $\lambda_{j}$ tal que $\lambda_{j}^{2} A_{j}\left\|v_{j}\right\|_{L^{\infty}}=1$, onde $A_{j}$ foi definido no início da seção. Suponha que existam subsequências com $\left\|u_{j}\right\|_{L^{\infty}} \leq\left\|v_{j}\right\|_{L^{\infty}}$ e sejam:

$$
\tilde{u}_{j}(x)=\frac{u_{j}\left(\lambda_{j} \epsilon_{j} x+y_{j}\right)}{\left\|v_{j}\right\|_{L^{\infty}}} \text { e } \quad \tilde{v}_{j}(x)=\frac{v_{j}\left(\lambda_{j} \epsilon_{j} x+y_{j}\right)}{\left\|v_{j}\right\|_{L^{\infty}}}
$$

que satisfazem o sistema

$$
\left\{\begin{aligned}
-\Delta \tilde{u}_{j}+\lambda_{j}^{2} \tilde{u}_{j}=\frac{\lambda_{j}^{2}}{\left\|v_{j}\right\|_{L^{\infty}}} g_{j}\left(\left\|v_{j}\right\|_{L^{\infty}} \tilde{v}_{j}\right) & \text { em } \Omega_{j} \\
-\Delta \tilde{v}_{j}+\lambda_{j}^{2} \tilde{v}_{j}=\frac{\lambda_{j}^{2}}{\left\|v_{j}\right\|_{L^{\infty}}} f\left(\left\|v_{j}\right\|_{L^{\infty}} \tilde{u}_{j}\right) & \text { em } \Omega_{j}
\end{aligned}\right.
$$

e são tais que $\lambda_{j} \rightarrow 0,\left\|\tilde{u}_{j}\right\|_{L^{\infty}} \leq 1$ e $\left\|\tilde{v}_{j}\right\|_{L^{\infty}}=1$.

Note que usando (2.3) vemos que $\frac{\lambda_{j}^{2}}{\left\|v_{j}\right\|_{L^{\infty}}} g_{j}\left(\left\|v_{j}\right\|_{L^{\infty}} \tilde{v}_{j}\right) \rightarrow|v|^{p-2} v$ e também que $\frac{\lambda_{j}^{2}}{\left\|v_{j}\right\|_{L^{\infty}}} f\left(\left\|v_{j}\right\|_{L^{\infty}} \tilde{u}_{j}\right) \rightarrow 0$ quando $j \rightarrow \infty$.

Logo o limite $(\bar{u}, \bar{v})$ de $\left(\tilde{u}_{j}, \tilde{v}_{j}\right)$ satisfaz o seguinte problema limite:

$$
\left\{\begin{array}{llll}
-\Delta \bar{u}_{j} & =|\bar{v}|^{p-2} \bar{v} & & \text { em } \omega \\
-\Delta \bar{v} & =0 & & \text { em } \omega
\end{array} .\right.
$$

Pela Proposição 2.2.5 item (1), temos agora que $\bar{v}=0$, o que contraria o fato de $\left|\bar{v}_{j}(0)\right|=\left|\tilde{v}_{j}(0)\right|=1, \forall j \in \mathbb{N}$.

CASO 3 - Tratemos agora do caso em que $l=0$ e $\left\|v_{j}\right\|_{L^{\infty}} \leq\left\|u_{j}\right\|_{L^{\infty}}$. Suponha que:

$$
\frac{\left\|v_{j}\right\|_{L^{\infty}}}{\left\|u_{j}\right\|_{L^{\infty}}} A_{j}^{\frac{1}{p}} \longrightarrow \infty
$$

Seja

$$
\tilde{u}_{j}(x)=\frac{u_{j}\left(\lambda_{j} \epsilon_{j} x+y_{j}\right)}{\left\|u_{j}\right\|_{L^{\infty}}} \quad \text { e } \quad \tilde{v}_{j}(x)=\frac{v_{j}\left(\lambda_{j} \epsilon_{j} x+y_{j}\right)}{\left\|v_{j}\right\|_{L^{\infty}}}
$$

e $\lambda_{j}^{2}=\frac{\left\|u_{j}\right\|_{L} \infty}{A_{j}\left\|u_{j}\right\|_{L \infty}^{p-1}}$. Note que $\lambda_{j} \rightarrow 0^{+}$, pois $\lambda_{j}^{-2}=A_{j}^{\frac{p-1}{p}}\left\|v_{j}\right\|^{p-2}\left(\frac{A_{j}^{\frac{1}{p}}\left\|v_{j}\right\|}{\left\|u_{j}\right\|}\right) \rightarrow \infty$. Da mesma forma que no Caso 2, chegamos ao problema limite:

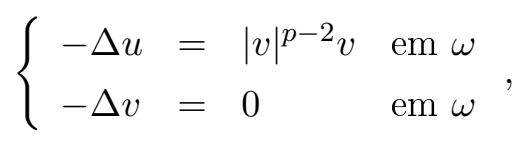

o que implicará pela Proposição 2.2.5 item (1) que $v=0$, o que contraria o fato de $\left|\tilde{v}_{j}(0)\right|=1, \forall j \in \mathbb{N}$.

CASO 4 - O único caso que resta considerar é quando $l=0,\left\|v_{j}\right\|_{L^{\infty}} \leq\left\|u_{j}\right\|_{L^{\infty}}$, porém não vale que $\frac{\left\|v_{j}\right\|_{L^{\infty}}}{\left\|u_{j}\right\|_{L^{\infty}}} A_{j}^{\frac{1}{p}} \rightarrow \infty$, ou seja, existe uma constante $C$ tal que, para todo $j \in \mathbb{N}$, $\left\|v_{j}\right\|_{L^{\infty}} A_{j}^{\frac{1}{p}} \leq C\left\|u_{j}\right\|_{L^{\infty}}$. Nesse caso, seja:

$$
\tilde{u}_{j}(x)=\frac{u_{j}\left(\lambda_{j} \epsilon_{j} x+x_{j}\right)}{\left\|u_{j}\right\|_{L^{\infty}}}, \tilde{v}_{j}(x)=\frac{v_{j}\left(\lambda_{j} \epsilon_{j} x+x_{j}\right)}{\left\|v_{j}\right\|_{L^{\infty}}} \quad \text { e } \quad \lambda_{j}^{2}=\frac{\left\|v_{j}\right\|_{L^{\infty}}}{\left\|u_{j}\right\|_{L^{\infty}}^{p-1}}
$$


e $x_{j}$ é tal que $\left|u_{j}\left(x_{j}\right)\right|=\left\|u_{j}\right\|_{L^{\infty}}$. Note que $\lambda_{j}^{2} \leq \frac{\left\|u_{j}\right\|_{L^{\infty}}}{\left\|u_{j}\right\|_{L^{\infty}}^{p-1}}=\frac{1}{\left\|u_{j}\right\|_{L^{\infty}}^{p-2}} \rightarrow 0$.

A menos de subsequência, seja:

$$
\bar{\lambda}=\lim _{j \rightarrow \infty} \frac{\lambda_{j}^{2} A_{j}\left\|v_{j}\right\|_{L^{\infty}}^{p-1}}{\left\|u_{j}\right\|_{L^{\infty}}}
$$

e note que $0 \leq \bar{\lambda} \leq \infty$, pois, como $\lim \frac{A_{j}^{\frac{1}{p}}\left\|v_{j}\right\|_{L^{\infty}}}{\left\|u_{j}\right\|_{L^{\infty}}}<\infty$, então

$$
\bar{\lambda}=\lim \frac{\left\|v_{j}\right\|_{L^{\infty}}}{\left\|u_{j}\right\|_{L^{\infty}}^{p-1}} \frac{\left\|v_{j}\right\|_{L^{\infty}}^{p-1}}{\left\|u_{j}\right\|_{L^{\infty}}} A_{j}=\lim \left(A_{j}^{\frac{1}{p}} \frac{\left\|v_{j}\right\|_{L^{\infty}}}{\left\|u_{j}\right\|_{L^{\infty}}}\right)^{p}<\infty .
$$

Note que $\frac{\lambda_{j}^{2}}{\left\|u_{j}\right\|_{L^{\infty}}} g_{j}\left(\left\|v_{j}\right\|_{L^{\infty}} \tilde{v}_{j}\right) \rightarrow \bar{\lambda}|v|^{p-2} v$ e também $\frac{\lambda_{j}^{2}}{\left\|v_{j}\right\|_{L^{\infty}}} f\left(\left\|u_{j}\right\|_{L^{\infty}} \tilde{u}_{j}\right) \rightarrow \frac{l_{1}}{p-1}|u|^{p-2} u$. Então $(u, v)$ satisfaz o problema limite:

$$
\left\{\begin{array}{rlrl}
-\Delta u & =(p-1) \bar{\lambda}|v|^{p-2} v & & \text { em } \omega \\
-\Delta v & =\frac{l_{1}}{p-1}|u|^{p-2} u & & \text { em } \omega
\end{array} .\right.
$$

Então se $\bar{\lambda}>0$, a Proposição 2.2.5 item (2) implica que $u=v=0$, pois $(p-1) \bar{\lambda}|s|^{p-2} s$ satisfaz (a), (b) e (c). Se $\bar{\lambda}=0$, então o item (1) implica que $u=0$. Logo podemos afirmar em qualquer dos casos que $u=0$, o que contradiz o fato de $\left|\tilde{u}_{j}(0)\right|=1, \forall j \in \mathbb{N}$.

Seja $\epsilon_{j} \rightarrow 0$ uma sequência qualquer e $u_{j}, v_{j}$ solução do problema $(2.1) \operatorname{com} \epsilon=\epsilon_{j}$, tal que $m\left(u_{j}, v_{j}\right)$ é limitado uniformemente. Pelo Teorema 2.2.1, segue que $\left\|u_{j}\right\|_{L^{\infty}}$ e $\left\|v_{j}\right\|_{L^{\infty}}$ são limitados. Seja $x_{j} \in \bar{\Omega}$ e consideremos agora o seguinte reescalonamento das soluções:

$$
\tilde{u}_{j}(x)=u_{j}\left(\epsilon_{j} x+x_{j}\right) \quad \text { e } \quad \tilde{v}_{j}(x)=v_{j}\left(\epsilon_{j} x+x_{j}\right)
$$

que satisfaz o seguinte sistema:

$$
\left\{\begin{array}{lll}
-\Delta \tilde{u}_{j}+\tilde{u}_{j}=g\left(\tilde{v}_{j}\right) & \text { em } \Omega_{j} \\
-\Delta \tilde{v}_{j}+\tilde{v}_{j}=f\left(\tilde{u}_{j}\right) & \text { em } \Omega_{j} \\
\frac{\partial \tilde{u}_{j}}{\partial n}=\frac{\partial \tilde{v}_{j}}{\partial n}=0 & \text { sobre } \partial \Omega_{j}
\end{array}\right.
$$

onde $\Omega_{j}=\frac{1}{\epsilon_{j}}\left(\Omega-x_{j}\right)$. Devido as estimativas que temos sobre $\tilde{u}_{j}$ e $\tilde{v}_{j}$, vemos que, assim como na demonstração do Teorema 2.2.1, $\tilde{u}_{j} \rightarrow u$ e $\tilde{v}_{j} \rightarrow v$ em $C_{l o c}^{2}$ a menos de subsequência, onde $u$ e $v$ satisfazem:

$$
\left\{\begin{array}{lll}
-\Delta u+u & =g(v) & \text { em } \omega \\
-\Delta v+v & =f(u) & \text { em } \omega \\
\frac{\partial u}{\partial n}=\frac{\partial v}{\partial n}=0 & & \text { sobre } \partial \omega
\end{array}\right.
$$

onde $\omega=\mathbb{R}^{N}$ ou, no caso em que $x_{j} \rightarrow x_{0} \in \partial \Omega, \omega=\left\{x ;\left\langle x, n\left(x_{0}\right)\right\rangle<d_{0}\right\}$ e $d_{0}=$ $\lim _{j \rightarrow \infty} \frac{\operatorname{dist}\left(x_{j}, \partial \Omega\right)}{\epsilon_{j}}$. 
Mostra-se exatamente da mesma forma que no Teorema 2.2.1, que $(u, v)$ tem índice finito. Usando este fato, juntamente com a hipótese (2.4), mostra-se como na demonstração do item (2) da Proposição 2.2.5 que $u, v \in H^{1}(\omega)$. Isto implica que

$$
I_{\infty}(u, v)=\int_{\omega}(\langle\nabla u, \nabla v\rangle+u v-F(u)-G(v))<\infty
$$

O próximo resultado é no sentido de fornecer condições suficientes para a convergência:

$$
I_{j}\left(\tilde{u}_{j}, \tilde{v}_{j}\right)=\int_{\Omega_{j}}\left(\left\langle\nabla \tilde{u}_{j}, \nabla \tilde{v}_{j}\right\rangle+\tilde{u}_{j} \tilde{v}_{j}-F\left(\tilde{u}_{j}\right)-G\left(\tilde{v}_{j}\right)\right) \longrightarrow I_{\infty}(u, v) .
$$

Como $\omega$ é ilimitado e a convergência de $\tilde{u}_{j}$ e $\tilde{v}_{j}$ é sobre compactos, então a convergência de $I_{j}\left(\tilde{u}_{j}, \tilde{v}_{j}\right)$ para $I_{\infty}(u, v)$ não segue diretamente.

Proposição 2.2.6. Suponha verdadeira a hipótese $(H)$ e seja $u_{j}$ e $v_{j}$ tais que $m\left(u_{j}, v_{j}\right) \leq 1$ $e(u, v) \neq(0,0)$. Então para todo $\delta>0$, existe $R>0$ e $j_{0} \in \mathbb{N}$ tais que:

$$
\int_{\Omega_{j} \cap\{x ;|x| \geq R\}}\left(f\left(\tilde{u}_{j}\right) \tilde{u}_{j}+g\left(\tilde{v}_{j}\right) \tilde{v}_{j}\right) \leq \delta \quad \forall j \geq j_{0} .
$$

Demonstração. Não podemos ter que $\forall \varphi \in C_{0}^{\infty}(\omega)$, vale as duas seguintes desigualdades:

$$
I_{\infty}^{\prime \prime}(u, v)(u \varphi, u \varphi)^{2} \geq 0 \quad \text { e } \quad I_{\infty}^{\prime \prime}(u, v)(v \varphi, v \varphi)^{2} \geq 0
$$

pois se valessem, argumentando da mesma forma que na Proposição 2.2.5, existiria uma constante $C$ independente de $\varphi$, tal que:

$$
\int_{B_{2 R}}\left(|\nabla u|^{2}+|\nabla v|^{2}+u^{2}+v^{2}+f(u) u+g(v) v\right) \varphi^{2} \leq C \int_{\omega}\left(u^{2}+v^{2}\right)|\nabla \varphi|^{2}<\frac{c}{R^{2}} \longrightarrow 0,
$$

quando $R \rightarrow 0$, onde $\varphi=1$ em $B_{R}, \varphi=0$ em $B_{2 R}^{C}$ e $|\nabla \varphi|<\frac{C}{R}$. Mas isso implica que $u=v=0 \mathrm{em} \omega$. Logo deve existir uma $\varphi_{1}$ com suporte em uma bola $B_{R_{1}}$ com, digamos,

$$
I_{\infty}^{\prime \prime}(u, v)\left(u \varphi_{1}, u \varphi_{1}\right)^{2}<0
$$

Como $\tilde{u}_{j} \rightarrow u$ e $\tilde{v}_{j} \rightarrow v$ em $C_{l o c}^{2}$ e $I_{j}^{\prime \prime}\left(\tilde{u}_{j}, \tilde{v}_{j}\right)\left(u \varphi_{1}, u \varphi_{1}\right)^{2}$ é uma integral calculada sobre $\overline{B_{R_{1}}}$ que é compacto, então

$$
I_{j}^{\prime \prime}\left(\tilde{u}_{j}, \tilde{v}_{j}\right)\left(u \varphi_{1}, u \varphi_{1}\right)^{2} \rightarrow I_{\infty}^{\prime \prime}(u, v)\left(u \varphi_{1}, u \varphi_{1}\right)^{2}<0
$$

o que significa que para $j$ suficientemente grande, $I_{j}^{\prime \prime}\left(\tilde{u}_{j}, \tilde{v}_{j}\right)\left(u \varphi_{1}, u \varphi_{1}\right)^{2}<0$. Como $m\left(u_{j}, v_{j}\right) \leq 1$ e $u \varphi_{1}$ é uma função tal que para $j$ suficientemente grande, $I_{j}^{\prime \prime}\left(\tilde{u}_{j}, \tilde{v}_{j}\right)\left(u \varphi_{1}, u \varphi_{1}\right)^{2}<0$, a forma contrapositiva do Lema 2.2.3 garante que toda $\Psi \in H^{1}\left(\Omega_{j}\right)$ com suporte disjunto do suporte de $u \varphi_{1}$, é tal que $I_{j}^{\prime \prime}\left(\tilde{u}_{j}, \tilde{v}_{j}\right)(\Psi, \Psi)^{2} \geq 0$, em particular as $\Psi$ que se anulam em $B_{R_{1}}(0)$. 
Dado $\delta>0$, escolha $R>0$ grande o suficiente para que

$$
\int_{B_{2 R} \backslash B_{R} \cap \omega}\left(u^{2}+v^{2}\right)<\delta .
$$

Dessa forma, se $j \in \mathbb{N}$ é grande o suficiente, $\int_{\Omega_{j} \cap\left(B_{2 R} \backslash B_{R}\right)}\left(\tilde{u}_{j}^{2}+\tilde{v}_{j}^{2}\right)<\delta$. Seja então $\varphi$ uma função de corte tal que $\varphi=0$ em $B_{R}$ e $\varphi=1$ em $B_{2 R}^{C}$. Tomando $\Psi=\varphi \tilde{u}_{j}$ e $\Psi=\varphi \tilde{v}_{j}$, temos $I_{j}^{\prime \prime}\left(\tilde{u}_{j}, \tilde{v}_{j}\right)(\Psi, \Psi)^{2} \geq 0$ e, argumentando novamente como na Proposição 2.2.5, teremos:

$$
\int_{\Omega_{j} \cap B_{2 R}^{C}}\left(\left|\nabla \tilde{u}_{j}\right|^{2}+\left|\nabla \tilde{v}_{j}\right|^{2}+\tilde{u}_{j}^{2}+\tilde{v}_{j}^{2}+f\left(\tilde{u}_{j}\right) \tilde{u}_{j}+g\left(\tilde{v}_{j}\right) \tilde{v}_{j}\right) \leq C \delta .
$$

Isto implica diretamente na tese do teorema.

Observação 2.2.7. Observe que como $f^{\prime}(s) \geq 0, \forall s \in \mathbb{R}$ então $|F(s)| \leq \int_{0}^{s}|f(t)| d t \leq f(s) s$. Multiplicando as equações do sistema (2.26) por $\tilde{u}_{j}$ e $\tilde{v}_{j}$, respectivamente, obtemos também que

$$
\int_{\omega}\left(\left\langle\nabla \tilde{u}_{j}, \nabla \tilde{v}_{j}\right\rangle+\tilde{u}_{j} \tilde{v}_{j}\right)=\int_{\omega} f\left(\tilde{u}_{j}\right) \tilde{u}_{j}=\int_{\omega} g\left(\tilde{v}_{j}\right) \tilde{v}_{j}
$$

Esses dois fatos e mais a Proposição 2.2.6 implicam na convergência de $I_{j}\left(\tilde{u}_{j}, \tilde{v}_{j}\right)$ para $I_{\infty}(u, v)$.

Observação 2.2.8. Observamos que, se $u_{j}>0$ e $v_{j}>0$ em $\Omega$, então

$$
\liminf _{j \rightarrow \infty} \min \left\{\left\|u_{j}\right\|_{\infty},\left\|v_{j}\right\|_{\infty}\right\}>0
$$

Isto segue observando que, como $u_{j}$ e $v_{j}$ satisfazem o sistema (2.1), então $w_{j}=u_{j}+v_{j}$ satisfaz a equação

$$
-\epsilon_{j}^{2} \Delta w_{j}=u_{j}\left(\frac{f\left(u_{j}\right)}{u_{j}}-1\right)+v_{j}\left(\frac{g\left(v_{j}\right)}{v_{j}}-1\right) .
$$

Não pode ocorrer que $-\Delta w_{j}<0$ em uma vizinhança de um ponto de máximo de $w_{j}$ e isto se estende até a fronteira devido ao Lema de Hopf. Porém, observando que a hipótese (2.3) implica que $f$ e $g$ são $o(s)$ quando $s \rightarrow 0$, então para valores pequenos de $u_{j}+v_{j}$, o lado direito de (2.30) é negativo. Dessa forma concluímos que, digamos, $\liminf \operatorname{in}_{j \rightarrow \infty}\left\|u_{j}\right\|_{L^{\infty}}>0$. Como $-\epsilon_{j}^{2} \Delta u_{j}=g\left(v_{j}\right)-u_{j}$, pelo mesmo motivo segue que $g\left(v_{j}\left(x_{j}\right)\right) \geq u_{j}\left(x_{j}\right)=\left\|u_{j}\right\|_{L^{\infty}}$, de maneira que $\liminf \operatorname{in}_{j \rightarrow \infty}\left\|v_{j}\right\|_{L^{\infty}}>0$.

\subsection{Forma das soluções positivas}

Nessa seção, iremos estudar algumas características interessantes das soluções do problema (2.1), para valores de $\epsilon$ suficientemente pequenos. Para isto iremos admitir a hipótese $(H)$, bem como uma limitação uniforme no Índice de Morse das soluções. 
Teorema 2.3.1. Suponha que valha $(H)$ e sejam $\left(u_{\epsilon}, v_{\epsilon}\right)$ solução positiva de (2.1) com $m\left(u_{\epsilon}, v_{\epsilon}\right) \leq 1$. Suponha que exista $\epsilon_{1}>0$ e $C>0$ tal que para todo $0<\epsilon \leq \epsilon_{1}$, $u_{\epsilon}$ tem um ponto de máximo satisfazendo:

$$
\operatorname{dist}\left(x_{\epsilon}, \partial \Omega\right) \leq C \epsilon
$$

Então existe $\epsilon_{0}>0$ tal que se $0<\epsilon<\epsilon_{0}, x_{\epsilon}$ é o único ponto de máximo de $u_{\epsilon}$ e também de $v_{\epsilon}$, além disso $x_{\epsilon} \in \partial \Omega$.

Demonstração. Note que é suficiente mostrar que toda sequência de soluções de (2.1) com $\epsilon=\epsilon_{j} \rightarrow 0$ tem uma subsequência satisfazendo a tese do teorema, ou seja, que toda sequência possui uma subsequência que, a partir de um certo $\epsilon_{0}$, possui um único e comum ponto de máximo localizado em $\partial \Omega$.

Denotaremos por $u_{j}, v_{j}$ e $x_{j}$ subsequências que serão extraídas de $u_{\epsilon_{j}}, v_{\epsilon_{j}}$ e $x_{\epsilon_{j}}$, respectivamente. Vamos dividir a prova em vários passos:

1. A hipótese de que existem $\epsilon_{1}, C>0$ tais que $\operatorname{dist}\left(x_{\epsilon_{j}}, \partial \Omega\right) \leq C \epsilon_{j}$, para todo $\epsilon_{j} \in\left(0, \epsilon_{1}\right)$ e a compacidade de $\partial \Omega$, implicam que a menos de subsequência, $x_{\epsilon_{j}}$ converge para um ponto de $\partial \Omega$. . Por isso, podemos afirmar que, a menos de subsequência, $x_{j} \rightarrow P \in \partial \Omega$. Para mostrar que, para $j$ suficientemente grande, $x_{j}=P$, vamos provar primeiramente que

$$
d_{0}=\lim _{j \rightarrow \infty} \frac{\operatorname{dist}\left(x_{j}, \partial \Omega\right)}{\epsilon_{j}}=0
$$

Definamos $\tilde{u}_{j}(x)=u_{j}\left(\epsilon_{j} x+x_{j}\right)$ e $\tilde{v}_{j}(x)=v_{j}\left(\epsilon_{j} x+x_{j}\right)$ onde $x_{j}$ é ponto de máximo de $u_{j}$. Suponhamos, sem perda de generalidade, que $n(P)=e_{N}$ e denotemos por $x_{N}=\left\langle e_{n}, x\right\rangle$. Sabemos que as sequências $\tilde{u}_{j}$ e $\tilde{v}_{j}$ convergem em $C_{l o c}^{2}$ para $u$ e $v$, respectivamente, definido sobre $\omega=\left\{x \in \mathbb{R}^{N} ;\langle x, n(P)\rangle<d_{0}\right\}=\left\{x ; x_{N}<d_{0}\right\}$. Como $u_{j}$ e $v_{j}$ são positivas por hipótese, (2.29) implica que $\|u\|_{L^{\infty}} \geq \liminf \left\|u_{j}\right\|_{L^{\infty}}>0$ e analogamente para $v$, logo, $u(0)>0$ e $v(0)>0$.

Consideremos o hiperplano $\left\{x ; x_{N}=d_{0}\right\}$ e vamos refletir $u$ e $v$ (que são definidas em $\left.\omega=\left\{x ; x_{N}<d_{0}\right\}\right)$ com relação a este. Dessa forma, $u$ e $v$ satisfazem o seguinte sistema:

$$
\left\{\begin{aligned}
-\Delta u+u=g(v) & \text { em } \mathbb{R}^{N} \\
-\Delta v+v & =f(u) \quad \text { em } \mathbb{R}^{N}
\end{aligned}\right.
$$

e estão em $H^{1}\left(\mathbb{R}^{N}\right)$. Segue pelo fato de $u, v \in H^{1}(\Omega)$ que $u$ e $v$ têm um decaimento no infinito, ou seja, $\lim _{|x| \rightarrow \infty} u(x)=0$ e $\lim _{|x| \rightarrow \infty} v(x)=0$. Por [3], segue que, como $f(0)=f^{\prime}(0)=0$ e $f^{\prime} \geq 0$ e analogamente para $g, u$ e $v$ são radialmente simétricas com respeito ao seu único ponto de máximo $x_{0} \in \mathbb{R}^{N}$. Como por construção $u(0)=\max _{\mathbb{R}^{N}} u$, então $x_{0}=0$. Logo, como $u$ e $v$ são simétricas com relação a 0 e também ao hiperplano $\left\{x ; x_{N}=d_{0}\right\}$, necessariamente $d_{0}=0$. 
2. Seja agora $y_{j}$ um ponto de máximo de $v_{j}$ em $\bar{\Omega}$. Mostremos que existe $C>0$ tal que, para $j$ grande o suficiente

$$
\left|x_{j}-y_{j}\right| \leq C \epsilon_{j}
$$

Definamos por $\bar{u}_{j} \rightarrow \bar{u}$ e $\bar{v}_{j} \rightarrow \bar{v}$ a sequência de blow-up, onde $\bar{u}_{j}(x)=u_{j}\left(\epsilon_{j} x+y_{j}\right)$ e $\bar{v}_{j}(x)=v_{j}\left(\epsilon_{j} x+y_{j}\right)$. Pelo mesmo motivo que $u$, agora $\bar{v}(0)>0$. Pela Proposição 2.2.6, para todo $\delta>0$, existe $R>0$ tal que, para $j$ suficientemente grande

$$
\int_{\bar{\Omega}_{j} \backslash B_{R}(0)} f\left(\bar{u}_{j}\right) \bar{u}_{j}+g\left(\bar{v}_{j}\right) \bar{v}_{j} \leq \delta
$$

onde $\bar{\Omega}_{j}=\frac{1}{\epsilon_{j}}\left(\Omega-y_{j}\right)$. Denotando por $z_{j}=\frac{y_{j}-x_{j}}{\epsilon_{j}}$, como $\tilde{u}_{j}\left(z_{j}\right)=u_{j}\left(y_{j}\right)=\bar{u}_{j}(0)$, então a desigualdade anterior lê-se como

$$
\int_{\Omega_{j} \backslash B_{R}\left(z_{j}\right)} f\left(\tilde{u}_{j}\right) \tilde{u}_{j}+g\left(\tilde{v}_{j}\right) \tilde{v}_{j} \leq \delta .
$$

Se assumirmos por contradição que $\left|z_{j}\right| \rightarrow \infty$, então, para todo $\delta>0$, existe $j_{0}$ tal que

$$
\int_{\Omega_{j}} f\left(\tilde{u}_{j}\right) \tilde{u}_{j}+g\left(\tilde{v}_{j}\right) \tilde{v}_{j} \leq 2 \delta \quad \forall j>j_{0}
$$

pois se $\left|z_{j}\right|$ for muito grande, as bolas $B_{R}(0)$ e $B_{R}\left(z_{j}\right)$ serão disjuntas, de modo que, pela Proposição 2.2.6 e por (2.34), teremos

$\int_{\Omega_{j}} f\left(\tilde{u}_{j}\right) \tilde{u}_{j}+g\left(\tilde{v}_{j}\right) \tilde{v}_{j} \leq \int_{\Omega_{j} \backslash B_{R}(0)} f\left(\tilde{u}_{j}\right) \tilde{u}_{j}+g\left(\tilde{v}_{j}\right) \tilde{v}_{j}+\int_{\Omega_{j} \backslash B_{R}\left(z_{j}\right)} f\left(\tilde{u}_{j}\right) \tilde{u}_{j}+g\left(\tilde{v}_{j}\right) \tilde{v}_{j} \leq 2 \delta$

para todo $j>j_{0}$. Isso implica que $\int_{\omega}(f(u) u+g(v) v)=0$. Logo $u=v=0$, pois, por (2.4), $F(s)>0$ para $s>0, \operatorname{logo} f(s)>0$ quando $s>0$ e analogamente para $g$.

Esta contradição mostra que $\left|z_{j}\right|$ não converge para $\infty$ e, portanto, vale (2.33).

3. Queremos agora mostrar que, se $y_{j}$ é qualquer ponto de máximo de $v_{j}$ em $\bar{\Omega}$, então:

$$
\lim _{j \rightarrow \infty} \frac{\left|x_{j}-y_{j}\right|}{\epsilon_{j}}=0
$$

Note que, por (2.33), $\left(y_{j}\right)$ converge para o mesmo limite $P \in \partial \Omega$, pois dado $\delta>0$, tome $j_{0}$ grande de forma que $\left|x_{j}-P\right|<\frac{\delta}{2}$ para $j>j_{0}$, e também de maneira que $\left|x_{j}-y_{j}\right|<C \epsilon_{j}<\frac{\delta}{2}$ para $j>j_{0}$ (isto é possível pois $\epsilon_{j} \rightarrow 0$ ). Teremos então

$$
j>j_{0} \Rightarrow\left|y_{j}-P\right| \leq\left|y_{j}-x_{j}\right|+\left|x_{j}-P\right|<\delta .
$$

Segue por (2.32) que $\frac{\operatorname{dist}\left(y_{j}, \partial \Omega\right)}{\epsilon_{j}}$ é limitado, pois dado $\delta>0, \exists j_{0} \in \mathbb{N}$ tal que $j>j_{0}$ implica que

$$
\frac{\operatorname{dist}\left(y_{j}, \partial \Omega\right)}{\epsilon_{j}} \leq \frac{\left|y_{j}-x_{j}\right|}{\epsilon_{j}}+\frac{\operatorname{dist}\left(x_{j}, \partial \Omega\right)}{\epsilon_{j}}<C+\delta .
$$


Como temos um resultado como (2.31) também para $y_{j}$, podemos prosseguir como no primeiro passo, definindo sequências de funções $\bar{u}_{j}$ e $\bar{v}_{j}$ com $y_{j}$ em lugar de $x_{j}$, chegando a conclusão de que $\lim _{j \rightarrow \infty} \frac{\operatorname{dist}\left(y_{j}, \partial \Omega\right)}{\epsilon_{j}}=0$. Logo $\bar{u}_{j}$ e $\bar{v}_{j}$ convergem para $\bar{u}$ e $\bar{v}$ que estão definidas também em $\omega=\left\{x ; x_{N}<0\right\}$ e também atingem seus únicos máximos em 0 .

Como no segundo passo mostramos que $\left|z_{j}\right|$ não tende ao infinito, seja então $z_{0}=\lim z_{j}$. Então $\bar{u}_{j}(x)=u_{j}\left(\epsilon_{j} x+y_{j}\right)=\tilde{u}_{j}\left(x+\frac{y_{j}-x_{j}}{\epsilon_{j}}\right) \rightarrow u\left(x+z_{0}\right)$ pontualmente. Dessa forma $\bar{u}(x)=u\left(x+z_{0}\right)$ e $u$ terá máximo em $z_{0}$ (já que $\bar{u}$ tem em 0 ). Como $u$ tem seu único máximo em 0 , então $z_{0}=0$ e vale (2.35). Com isso vemos também que $\bar{u}=u$, ou seja, $\tilde{u}_{j}(x)=u_{j}\left(\epsilon_{j} x+x_{j}\right)$ e $\bar{u}_{j}(x)=u_{j}\left(\epsilon_{j} x+y_{j}\right)$ tem o mesmo limite.

4. Vamos agora usar (2.32) para mostrar que $x_{j} \in \partial \Omega$ para $j$ suficientemente grande e usar (2.35) e o fato de $\bar{u}_{j} \rightarrow u$ e $\bar{v}_{j} \rightarrow v$ em $C_{l o c}^{2}(\bar{\omega})$ para mostrar que $y_{j}=x_{j}$ para $j$ suficientemente grande.

Observe que, como $u$ e $v$ são radialmente simétricas com relação a origem, então considerando $u$ e $v$ como funções de $r=|x|$, temos que elas satisfazem o seguinte sistema:

$$
\left\{\begin{array}{lll}
-\left(r^{N-1} u^{\prime}\right)^{\prime} & = & r^{N-1}(g(v)-u) \\
-\left(r^{N-1} v^{\prime}\right)^{\prime} & = & r^{N-1}(f(u)-v) \\
-N u^{\prime \prime}(0) & = & g(v(0))-u(0) \\
-N v^{\prime \prime}(0) & = & f(u(0))-v(0)
\end{array} .\right.
$$

Como elas são funções não constantes, a versão local do Teorema de unicidade para soluções de EDO's implica que $u^{\prime \prime}(0) \neq 0$ ou $v^{\prime \prime}(0) \neq 0$. Suponhamos que seja $\left|u^{\prime \prime}(0)\right| \neq 0$, ou seja, $u$ tem um máximo não degenerado em 0 . Segue da convergência $C_{l o c}^{2}$ de $\tilde{u}_{j}$ para $u$, que é radialmente simétrica com relação a 0 , que para algum $\delta>0$ pequeno, $\left\langle\nabla u_{j}(x), y\right\rangle \neq 0$ para todo $x \in B_{\delta \epsilon_{j}}\left(x_{j}\right), x \neq x_{j}$ e para todo $y=\lambda\left(x-x_{j}\right)$, $\lambda \neq 0$. Porém, como $\frac{\partial u_{j}}{\partial n}=0$ em $\partial \Omega$, segue de (2.32) que $x_{j} \in \partial \Omega$.

Como $\bar{u}_{j}$ converge em $C_{l o c}^{2}$ para $u$, então existe um $\delta>0$ tal que não existe pontos críticos diferentes de $y_{j}$ dentro de $B_{\delta \epsilon_{j}}\left(y_{j}\right)$. Como por (2.35), $x_{j} \in B_{\delta \epsilon_{j}}\left(y_{j}\right)$ para valores de $j$ suficientemente grandes, então $x_{j}=y_{j}$.

\subsection{Estimativas de Energia}

Nessa seção vamos procurar estimativas superiores para a energia do sistema (2.1), de forma a podermos obter, através do Teorema 1.3.2, soluções com índice de Morse limitado. Isto possibilitará a aplicação de todos os resultados vistos até aqui neste capítulo. 
Teorema 2.4.1. Suponha $(H)$ com $2<p=q<2^{*}$ e seja (u,v) solução de (2.1) tal que $v \neq-u$. Então

$$
\sup _{E^{-} \oplus \mathbb{R}^{+}(u, v)} I=I(u, v)
$$

Demonstração. Vamos supor que $\epsilon=1$ para evitar mais tecnicalidades. Precisamos mostrar que para qualquer $\phi \in H^{1}(\Omega)$ e $t \geq 0, I((\phi,-\phi)+t(u, v)) \leq I(u, v)$. Isto é equivalente a mostrar que, fixado um $\phi \in H^{1}(\Omega)$ arbitrário, $\alpha(t)=I(t(u, v)+(1-t)(\phi,-\phi)) \leq I(u, v)=$ $\alpha(1)$. Na verdade vamos verificar que $\alpha$ tem em 1 um ponto de máximo. De fato, um dos pontos a ser verificado é que $\alpha(t) \rightarrow-\infty$ quando $t \rightarrow+\infty$. Seja $\bar{u}=\phi+t(u-\phi)$ e $\bar{v}=\psi+t(v-\psi)$, onde $\psi=-\phi$, então:

$$
\begin{aligned}
\alpha(t)= & \int_{\Omega}(\langle\nabla \bar{u}, \nabla \bar{v}\rangle+\bar{u} \bar{v}-F(\bar{u})-G(\bar{v})) \\
\leq & \|\nabla \bar{u}\|_{L^{2}}\|\nabla \bar{v}\|_{L^{2}}+\|\bar{u}\|_{L^{2}}\|\bar{v}\|_{L^{2}}-\int_{\Omega}(F(\bar{u})+G(\bar{v})) \\
\leq & \|\bar{u}\|_{H^{1}}\|\bar{v}\|_{H^{1}}-\int_{\Omega}(F(\bar{u})-G(\bar{v})) \\
\leq & \|\phi+t(u-\phi)\|_{H^{1}}\|\psi+t(v-\psi)\|_{H^{1}}-C \int_{\Omega}\left(|\bar{u}|^{p}+|\bar{v}|^{p}\right) \\
\leq & \left.t\left\|\frac{\phi}{t}-(u-\phi)\right\|_{H^{1}}\left\|\frac{\psi}{t}-(v-\psi)\right\|_{H^{1}}\left\|^{p}+\right\| \frac{\psi}{t}-(v-\psi) \|_{L^{p}}^{p}\right] \longrightarrow-\infty,
\end{aligned}
$$

quando $t \rightarrow+\infty$.

Precisamos mostrar que

$$
\alpha^{\prime}(1)=0 \quad \alpha^{\prime \prime}(1)<0 \quad \alpha(0) \leq 0 .
$$

- $\alpha^{\prime}(1)=0$ pois $\alpha^{\prime}(1)=I^{\prime}(u, v)(u-\phi, v-\Psi)=0$, já que $(u, v)$ é ponto crítico de $I$ por ser solução de (2.1);

- $\alpha(0) \leq 0$ pois $\alpha(0)=I(\phi,-\phi)=\int\left(-|\nabla \phi|^{2}-\phi^{2}-F(\phi)-G(-\phi)\right) \leq 0$ já que $F, G \geq 0$;

- para mostrar que $\alpha^{\prime \prime}(1)<0$, note que:

$$
\begin{aligned}
\alpha^{\prime \prime}(1)= & I^{\prime \prime}(u, v)(u-\phi, v+\phi)^{2} \\
= & \int_{\Omega}\left[2\langle\nabla(u-\phi), \nabla(v+\phi)\rangle+2(u-\phi)(v+\phi)-f^{\prime}(u)(u-\phi)^{2}-g^{\prime}(v)(v+\phi)^{2}\right] \\
= & \int_{\Omega}[2(\langle\nabla u, \nabla v\rangle+u v)+2(\langle\nabla u, \nabla \phi\rangle+u \phi)- \\
& \left.-2(\langle\nabla v, \nabla \phi\rangle+u \phi)-2\left(|\nabla \phi|^{2}+\phi^{2}\right)-f^{\prime}(u)(u-\phi)^{2}-g^{\prime}(v \mid)(v+\phi)^{2}\right] \\
= & -2\|\phi\|_{H^{1}}^{2}+i(f)+i(g) .
\end{aligned}
$$


onde

$$
i(f)=\int_{\Omega}\left(f(u) u-2 f(u) \phi-f^{\prime}(u)(u-\phi)^{2}\right) .
$$

Note que na expressão acima, multiplicamos as equações do sistema (2.1) por $u, v, \phi$, $-\phi$ e usamos integração por partes. Usando a hipótese a condição (2.4) da hipótese $(H)$, vemos que

$$
i(f) \leq-\delta \int_{\Omega} f(u) u<0 .
$$

Vamos agora definir uma função $\beta$ que irá coincidir com $\alpha$ nos seus pontos críticos e irá nos auxiliar na prova do teorema.

Seja $t$ tal que $\alpha^{\prime}(t)=0$, então, usando que $u-\phi=\frac{\bar{u}-\phi}{t}$ e analogamente para $v$ :

$$
\begin{aligned}
0= & \alpha^{\prime}(t)=I^{\prime}(\bar{u}, \bar{v})(u-\phi, v-\Psi) \\
= & \int_{\Omega}[\langle\nabla \bar{u}, \nabla(v-\Psi)\rangle+\langle\nabla \bar{v}, \nabla(u-\phi)\rangle+\bar{u}(v-\Psi)+\bar{v}(u-\phi)- \\
& -f(\bar{u})(u-\phi)-g(\bar{v})(v-\Psi)],
\end{aligned}
$$

o que implica que

$$
\int_{\Omega}(\langle\nabla \bar{u}, \nabla \bar{v}\rangle+\bar{u} \bar{v})=\frac{1}{2}\left[Q(\bar{u}, \Psi)+Q(\bar{v}, \phi)+\int(f(\bar{u}) t(u-\phi)+g(\bar{v}) t(v-\Psi))\right] .
$$

Definindo então $\beta(t)$ como:

$$
\beta(t)=\frac{1}{2}\left[Q(\bar{u}, \Psi)+Q(\bar{v}, \phi)+\int(f(\bar{u}) t(u-\phi)+g(\bar{v}) t(v-\Psi))\right]-\int_{\Omega}(F(\bar{u})-G(\bar{v})),
$$

temos as seguintes propriedades:

- $\beta(0) \leq 0$, pois $\beta(0)=\frac{1}{2}\left[Q(\phi, \Psi)+Q(\Psi, \phi)-\int_{\Omega}(F(\phi)-G(\Psi))\right]=I(\phi, \Psi)=\alpha(0) \leq 0$;

- $\beta(1)=\alpha(1)>0$ pois, por (2.4) $f^{\prime}(s) s^{2} \geq(1+\delta) f(s) s$, o que implica que $\int\left(f^{\prime}(s) s+f(s)\right) \geq \int(2+\delta) f(s)$. Assim $f(s) s \geq(2+\delta) F(s)$. Então

$$
\begin{aligned}
\alpha(1) & =I(u, v) \\
& =\int_{\Omega}\left[\frac{1}{2}(\langle\nabla u, \nabla v\rangle+u v)+\frac{1}{2}(\langle\nabla u, \nabla v\rangle+u v)-F(u)-G(v)\right] \\
& =\int_{\Omega}\left[\frac{1}{2} f(u) u-F(u)+\frac{1}{2} g(v) v-G(v)\right] \\
& \geq \int_{\Omega}\left(\frac{1}{2}-\frac{1}{2+\delta}\right)(f(u) u+g(v) v) \\
& >0
\end{aligned}
$$

- $\beta^{\prime}(1)>0$, pois através de cálculos simples vemos que

$$
\beta^{\prime}(1)=\|\phi\|_{H^{1}}^{2}-\frac{1}{2} i(f)-\frac{1}{2} i(g)=-\frac{\alpha^{\prime \prime}(1)}{2}>0 .
$$


Suponhamos por contradição que $\alpha$ tenha um máximo em $t_{0} \geq 0$ com $t_{0} \neq 1$. Então ou $t_{0} \in(0,1)$ ou $t_{0} \in(1,+\infty)$.

- se $t_{0} \in(0,1)$, então $\beta(0) \leq 0, \beta(1)>0$ e $\beta\left(t_{0}\right)>\beta(1)$, logo existe um ponto crítico de $\beta$ maior que 0 , ou seja:

$$
\exists \bar{t}>0 \quad \text { tal que } \quad \beta^{\prime}(\bar{t})=0 \text { e } \beta(\bar{t})>0 ;
$$

- se $t_{0} \in(1,+\infty)$, então como $0<1<t_{0}$ e $\alpha^{\prime}(t)=0, \alpha^{\prime \prime}(t)<0$ e $\alpha(1)<\alpha\left(t_{0}\right)$, como 1 é ponto de máximo, existe $t_{1} \in\left(1, t_{0}\right)$ com $\alpha^{\prime}\left(t_{1}\right)=0$ e $\alpha\left(t_{1}\right)<\alpha(1)$. Logo $\beta\left(t_{1}\right)=\alpha\left(t_{1}\right)<\alpha(1)=\beta(1)$. Como $\beta^{\prime}(1)>0$, então (2.36) vale.

Assim podemos afirmar em qualquer das situações que (2.36) vale. Para chegarmos até a contradição que encerrará a prova, mostremos que

$$
\forall t \geq 0 ; \quad \beta^{\prime}(t)=0 \Rightarrow \beta(t) \leq 0 .
$$

Note que isto contraria (2.36).

De fato, se $\beta^{\prime}(t)=0$, semelhantemente a $\alpha$, a parte quadrática na expressão de $\beta$ pode ser expressa em termos de $f$ e $g$, logo:

$$
\beta(t)=-\|\phi\|^{2}-\frac{\gamma(f)}{2}-\frac{\gamma(g)}{2} \text { onde } \gamma(f)=\int_{\Omega}\left(f^{\prime}(\bar{u}) t^{2}(u-\phi)^{2}-2 f(\bar{u}) t(u-\phi)+2 F(\bar{u})\right) .
$$
Por (2.4), $\gamma(f), \gamma(g) \geq 0$, o que implica que $\beta(t) \leq 0$.

Vamos apresentar uma generalização desse resultado que será utilizado na seção (2.5). Consideremos $u, v \in H^{1}\left(\mathbb{R}_{+}^{N}\right) \cap C^{2}\left(\mathbb{R}_{+}^{N}\right)$ solução de

$$
\left\{\begin{array}{rll}
-\Delta u+u & =g(v) & \text { em } \mathbb{R}_{+}^{N} \\
-\Delta v+v=f(u) & \text { em } \mathbb{R}_{+}^{N} \\
\frac{\partial u}{\partial n}=\frac{\partial v}{\partial n}=0 & \text { sobre } \partial \mathbb{R}_{+}^{N}
\end{array},\right.
$$

onde $\mathbb{R}_{+}^{N}=\left\{x \in \mathbb{R}^{N} ; x_{N}>0\right\}$. Vamos supor que $0 \in \partial \Omega$. Como $\Omega$ é um domínio $C^{2}$, seja $\alpha: \mathbb{R}^{N-1} \rightarrow \partial \Omega$ um $C^{2}$ difeomorfismo sobre uma vizinhança de 0 em $\partial \Omega$ tal que $\alpha(0)=0$ e $D \alpha(0)=I d$. Seja $\Phi(y, t)=\alpha(y)-t n(\alpha(y))$ onde $n(x)$ é o vetor unitário normal exterior a $\partial \Omega$ em $x \in \partial \Omega$. Note que, como $\frac{\partial \Phi}{\partial t}(0,0)=n(0)$ e $\frac{\partial \Phi}{\partial y}(0,0)=I d$, temos pelo Teorema da Função Inversa que $\Phi$ admite uma inversa local em 0, ou seja, existe $\Psi=\Phi^{-1}: B_{r}(0) \subset \mathbb{R}^{N} \rightarrow \mathbb{R}^{N-1} \times \mathbb{R}=\mathbb{R}^{N}$. Temos ainda que $D \Psi(x) n(x)=(0,1) \in \mathbb{R} \times \mathbb{R}$ para todo $x \in \partial \Omega$ próximo a 0 , pois:

$$
D \Psi(x) n(x)=\lim _{s \rightarrow 0} \frac{\Psi(x+s n(x))-\psi(x)}{s}=\lim _{s \rightarrow 0} \frac{\Psi(\Phi(y, s))-\Psi(\Phi(y, 0))}{s}=(0,1),
$$

onde $y=\alpha(x)$. Então $u \circ \Psi$ é tal que $\frac{\partial(u \circ \Psi)}{\partial n}=\nabla u(\Psi(x)) \cdot(0,1)=0$ em $\partial \Omega$.

Seja $\epsilon_{j} \rightarrow 0^{+}$e $\Omega_{j}=\frac{1}{\epsilon_{j}} \Omega$, definamos:

$$
u_{j}(x)=u\left(\frac{\Psi\left(\epsilon_{j} x\right)}{\epsilon_{j}}\right) \chi\left(\Psi\left(\epsilon_{j} x\right)\right) \in H^{1}\left(\Omega_{j}\right)
$$


onde $\chi \in C^{\infty}\left(\mathbb{R}^{N}\right), \chi=1$ em $B_{\frac{r}{2}}(0), \chi=0$ em $\mathbb{R}_{+}^{N} \backslash B_{r}(0), 0 \leq \chi \leq 1$ e $\chi$ é radialmente simétrica, em particular $\frac{\partial \chi}{\partial n}=0$ em $\partial \mathbb{R}_{+}^{N}$. Temos que $\frac{\partial u}{\partial n}=0$ em todo $x \in \partial \Omega_{j}$.

Da mesma forma como em (2.39) definimos $v_{j}$. O próximo Lema nos fornecerá algumas propriedades de $u_{j}$ e $v_{j}$.

Lema 2.4.2. Suponha que valha $(H)$ com $2<p=q<2^{*}$. Sejam $u, v \in H^{1}\left(\mathbb{R}_{+}^{N}\right) \cap C^{2}\left(\mathbb{R}_{+}^{N}\right)$, $v \neq-u \operatorname{com}(u, v)$ solução positiva de (2.38) e considere $u_{j}$ e $v_{j}$ como em (2.39). Então, quando $j \rightarrow+\infty$ e para $\phi_{j} \in H^{1}\left(\mathbb{R}_{+}^{N}\right)$ com $\left\|\phi_{j}\right\|_{H^{1}} \leq 1$ :

1. $\int_{\Omega_{j}}\left|u_{j}-u\right|^{p}=o(1)$;

2. $\int_{\Omega_{j}} f\left(u_{j}\right) \phi_{j}=\int_{\Omega_{j}} \frac{f\left(\bar{u}_{j}\right)}{\bar{u}_{j}} u_{j} \phi_{j}+o(1)$ se $\bar{u}_{j}=\phi_{j}+t_{j}\left(u_{j}-\phi_{j}\right)$, quando $t_{j} \rightarrow 1$.

Demonstração. $\quad$ 1. Dado $R>0$, note que a função $\left|u-u_{j}\right|^{p}$ é dominada em $B_{R}$ pela seguinte função $L^{1}\left(B_{R}\right),\left(2 \sup _{B_{R}} u\right)^{p}$, pois se $x \in B$, então

$$
\left|u(x)-u\left(\frac{\Psi\left(\epsilon_{j} x\right)}{\epsilon_{j}}\right) \chi\left(\Psi\left(\epsilon_{j} x\right)\right)\right|^{p} \leq 2^{p}\left(\sup _{B_{R}} u\right)^{p} .
$$

Logo, se mostrarmos que $u_{j} \rightarrow u$ pontualmente em $B_{R}$, teremos que $\int_{B_{R}}\left|u_{j}-u\right|^{p} \rightarrow 0$ quando $j \rightarrow+\infty$ pelo Teorema da Convergência Dominada de Lebesgue. Tomando $j$ suficientemente grande, temos $\Psi\left(\epsilon_{j} x\right) \in B_{\frac{r}{2}}(0)$. Logo,

$$
\left|u(x)-u_{j}(x)\right|=\left|u(x)-u\left(\frac{\Psi\left(\epsilon_{j} x\right)}{\epsilon_{j}}\right)\right|=\left|u\left(\frac{\Psi\left(\Phi\left(\epsilon_{j} x\right)\right)}{\epsilon_{j}}\right)-u\left(\frac{\Psi\left(\epsilon_{j} x\right)}{\epsilon_{j}}\right)\right| .
$$

Denotando por $h$ a função $x \mapsto \frac{x}{\epsilon_{j}}$, como $u \circ h \circ \Psi$ é contínua, dado $\epsilon>0, \exists \delta>0$ tal que se $\left|\Phi\left(\epsilon_{j} x\right)-\epsilon_{j} x\right|<\delta$, então $\left|u(x)-u_{j}(x)\right|<\epsilon$. Mas como $\Phi$ é contínua e $\epsilon_{j} \rightarrow 0$, então

$$
\lim _{j \rightarrow \infty}\left|\Phi\left(\epsilon_{j} x\right)-\epsilon_{j} x\right|=0 .
$$

Dessa forma existe $j_{0} \in \mathbb{N}$ tal que se $j>j_{0}$, então $\left|\Phi\left(\epsilon_{j} x\right)-\epsilon_{j} x\right|<\delta$, o que implica que $\left|u(x)-u_{j}(x)\right|<\epsilon$. Portanto $u_{j} \rightarrow u$ pontualmente. Então $\forall R>0, \int_{B_{R}}\left|u_{j}-u\right|^{p} \rightarrow 0$. Agora, como $u \in H^{1}\left(\mathbb{R}_{+}^{N}\right)$, então $\forall \epsilon>0, \exists R>0$ tal que $\int_{\mathbb{R}^{N} \backslash B_{R}}|u|^{p}<\epsilon$. Dessa forma o mesmo vale para $u_{j}$ com um $R^{\prime}>0$. Então

$$
\int_{\Omega_{j} \backslash B_{R}}\left|u-u_{j}\right|^{p} \leq\left[\left(\int_{\mathbb{R}^{N} \backslash B_{R}}|u|^{p}\right)^{\frac{1}{p}}+\left(\int_{\Omega_{j} \backslash B_{R}}\left|u_{j}\right|^{p}\right)^{\frac{1}{p}}\right]^{p}<\epsilon,
$$

ou seja, quando $R \rightarrow \infty$,

$$
\int_{\Omega_{j} \backslash B_{R}}\left|u-u_{j}\right|^{p} \rightarrow 0
$$

Portanto $\int_{\Omega_{j}}\left|u-u_{j}\right|^{p}=o(1)$ 
2. Temos que $u_{j}-\bar{u}_{j}=\lambda_{j}\left(\bar{u}_{j}-\phi_{j}\right)$ onde $\lambda_{j}=\frac{1-t_{j}}{t_{j}} \rightarrow 0$ e também que $\int_{\Omega_{j}}\left(f\left(u_{j}\right) \phi_{j}-\frac{f\left(\bar{u}_{j}\right)}{u_{j}} \phi_{j}\right)=\int_{\Omega_{j}}\left(f\left(u_{j}\right)-f\left(\bar{u}_{j}\right)\right) \phi_{j}-\int_{\Omega_{j}} \frac{f\left(\bar{u}_{j}\right)}{\bar{u}_{j}} \lambda_{j}\left(\bar{u}_{j}-\phi_{j}\right) \phi_{j}$. Usando o Teorema do Valor Médio e (2.3), ambas as integrais do lado direito da igualdade acima podem ser limitadas por

$$
C \lambda_{j} \int_{\Omega_{j}}\left(1+\left|u_{j}\right|^{p-2}+\left|\phi_{j}\right|^{p-2}\right)\left(\left|u_{j}\right|+\left|\phi_{j}\right|\right) \phi_{j} .
$$

Como $\phi_{j}$ é limitada em $L^{p}\left(\Omega_{j}\right)$, aplicando Hölder vemos que

$$
\int_{\Omega_{j}} f\left(u_{j}\right) \phi_{j}-\int_{\Omega_{j}} \frac{f\left(\bar{u}_{j}\right)}{\bar{u}_{j}} u_{j} \phi_{j}=o(1)
$$

Denotemos por $I_{j}$ o funcional energia associado a $u_{j}, v_{j}, \Omega_{j}$ e por $I_{\infty}$ o associado ao sistema (2.38).

Lema 2.4.3. Supondo que valha $(H)$ com $2<p=q<2^{*}$ e sendo $u, v \in H^{1}\left(\mathbb{R}_{+}^{N}\right) \cap C^{2}\left(\mathbb{R}_{+}^{N}\right)$, $u \neq-v$ soluções de (2.38) e $\phi_{j} \in H^{1}\left(\Omega_{j}\right)$ com $\left\|\phi_{j}\right\|=1$, então se $\left|t_{j}\right|+\left|s_{j}\right| \rightarrow \infty$, temos:

$$
I_{j}\left(t_{j}\left(\phi_{j},-\phi_{j}\right)+s_{j}\left(u_{j}, v_{j}\right)\right) \longrightarrow-\infty \text {. }
$$

Teorema 2.4.4. Suponha que valha $(H)$ com $2<p=q<2^{*}$. Sejam $u, v \in H^{1}\left(\mathbb{R}_{+}^{N}\right) \cap$ $C^{2}\left(\mathbb{R}_{+}^{N}\right), v \neq-u$ solução de (2.38) e sejam $u_{j}$ e $v_{j}$ como definido em (2.39). Então, quando $j \rightarrow \infty$ :

$$
\sup _{E^{-\oplus \mathbb{R}^{+}\left(u_{j}, v_{j}\right)}} I_{j}=I_{j}\left(u_{j}, v_{j}\right)+o(1)=I_{\infty}(u, v)+o(1) .
$$

Demonstração. Seja $t_{j}\left(\phi_{j},-\phi_{j}\right)+s_{j}\left(u_{j}, v_{j}\right)$ uma sequência maximizante para o supremo acima, onde $\left\|\phi_{j}\right\|=1, t_{j} \in \mathbb{R}$ e $s_{j} \geq 0$. Como $I_{j}\left(t_{j}\left(\phi_{j},-\phi_{j}\right)+s_{j}\left(u_{j}, v_{j}\right)\right) \rightarrow$ $\sup _{E^{-} \oplus \mathbb{R}^{+}\left(u_{j}, v_{j}\right)} I_{j} \geq 0$, pela contrapositiva do Lema 2.4.3, as sequências $\left(t_{j}\right)$ e $\left(s_{j}\right)$ são limitadas. Teremos dois casos a considerar:

- Suponhamos que $s_{j} \rightarrow 1$ e consideremos a função:

$$
\theta_{j}(t)=I_{j}\left(t\left(\phi_{j},-\phi_{j}\right)+s_{j}\left(u_{j}, v_{j}\right)\right), \quad t \in \mathbb{R}
$$

e seja $T_{j}$ um máximo de $\theta_{j}$. Vemos facilmente que $\theta_{j}^{\prime}(0) \rightarrow 0$. Note também que $T_{j} \rightarrow 0$. Com efeito, $\theta_{j}^{\prime}\left(T_{j}\right)=0$ e também:

$$
\begin{aligned}
\theta_{j}^{\prime \prime}(t) & =I_{j}^{\prime \prime}\left(t(\phi,-\phi)+s_{j}\left(u_{j}, v_{j}\right)\right)\left(\phi_{j},-\phi_{j}\right)^{2} \\
& =\int_{\Omega_{j}}\left(-2\left|\nabla \phi_{j}\right|^{2}-2\left|\phi_{j}\right|^{2}-f^{\prime}\left(\bar{u}_{j}\right) \phi_{j}^{2}-g^{\prime}\left(\bar{v}_{j}\right) \phi_{j}^{2}\right) \\
& =-2-\int_{\Omega_{j}}\left(f^{\prime}\left(\bar{u}_{j}\right) \phi_{j}^{2}+g^{\prime}\left(\bar{v}_{j}\right) \phi^{2}\right) \leq-2,
\end{aligned}
$$


para todo $j \in \mathbb{N}$ e $t \in \mathbb{R}$. Logo de fato $T_{j} \rightarrow 0$.

Como $\theta_{j}$ é contínua, segue que $\theta_{j}\left(T_{j}\right)=I_{j}\left(u_{j}, v_{j}\right)+o(1)$. Note agora que, como $T_{j}$ é máximo para $\theta_{j}$ e $t_{j}\left(\phi_{j},-\phi_{j}\right)+s_{j}\left(u_{j}, v_{j}\right)$ é uma sequência maximizante para o supremo, o resultado do enunciado segue.

- Suponhamos agora que $\liminf \left|s_{j}-1\right|>0$. Note que $t_{j} \phi_{j}+s_{j} u_{j}=\psi_{j}+s_{j}\left(u_{j}-\psi_{j}\right)$ onde $\psi_{j}=\frac{t_{j}}{1-s_{j}} \phi_{j}$. Seja

$$
\alpha_{j}(t)=I_{j}\left(t\left(u_{j}, v_{j}\right)+(1-t)\left(\phi_{j},-\phi_{j}\right)\right) .
$$

Semelhante ao Teorema 2.4.1, basta mostrar que para todo $\phi \in H^{1}\left(\Omega_{j}\right), \alpha_{j}(t) \leq$ $\alpha_{j}(1)+o(1) \forall t \geq 0$. Para isso, note que é suficiente que $t_{j} \rightarrow 1$, pois nesse caso, observando a expressão de $\alpha_{j}\left(t_{j}\right)-\alpha_{j}(1)$, vemos que isto tende a 0 quando $j \rightarrow \infty$. Suponha por contradição que $\liminf \left|t_{j}-1\right|>0$ e $\liminf \left|\alpha_{j}\left(t_{j}\right)-\alpha_{j}(1)\right|>0$. Note que chegando a uma contradição o teorema estará provado, pois o contrário de qualquer das condições acima implica no resultado. O resto da demonstração é muito parecida com a do Teorema 2.4.1. Usando a definição de $u_{j}$ e $v_{j}$ e o item (1) do Lema 2.4.2, verifica-se que $u_{j}$ e $v_{j}$ satisfazem o seguinte sistema

$$
\left\{\begin{array}{l}
-\Delta u_{j}+u_{j}=g\left(v_{j}+\mu_{j}(x) \text { em } \Omega_{j}\right. \\
-\Delta v_{j}+v_{j}=f\left(u_{j}\right)+\nu_{j}(x) \text { em } \Omega_{j}
\end{array},\right.
$$

onde $\mu_{j}$ e $\nu_{j}$ são tais que $\int_{\Omega_{j}}\left|\mu_{j}(x) \phi_{j}(x)\right| d x=o(1)$ e $\int_{\Omega_{j}}\left|\nu_{j}(x) \phi_{j}(x)\right| d x=o(1)$ quando $j \rightarrow \infty$.

Mostremos que:

- $\alpha_{j} \leq 0$ : segue como no Teorema 2.4.1;

- $\alpha_{j}^{\prime}(1)=o(1)$ : como $\alpha_{j}^{\prime}(1)=I_{j}^{\prime}\left(u_{j}, v_{j}\right)\left(u_{j}-\phi_{j}, v_{j}-\phi_{j}\right)$, então multiplicando a primeira equação do sistema (2.40) por $\left(u_{j}-\phi_{j}\right)$, a segunda por $\left(v_{j}-\Psi_{j}\right)$ e integrando por partes, segue que $\alpha_{j}^{\prime}(1)=o(1)$.

$-\sup _{|t-1|<\epsilon} \alpha^{\prime \prime}(t)<0$ : procedendo exatamente como no Teorema 2.4.1, chegamos a $\quad \alpha_{j}^{\prime \prime}(t) \quad-2\left\|\phi_{j}\right\|^{2}+i_{j}(f)+i_{j}(g) \quad$ onde $i_{j}(f)=\int_{\Omega_{j}}\left(f\left(u_{j}\right) u_{j}-2 f\left(u_{j}\right) \phi_{j}-f^{\prime}\left(\bar{u}_{j}\right)\left(u_{j}-\phi_{j}\right)^{2}\right)+o(1)$ e $\bar{u}_{j}=\phi_{j}+t\left(u_{j}-\phi_{j}\right)$ e analogamente para $g$. Por (2) do Lema 2.4.2, se $t$ está suficientemente próximo de 1:

$$
\begin{aligned}
i_{j}(f) & =\int_{\Omega_{j}}\left(\frac{f\left(\bar{u}_{j}\right)}{\bar{u}_{j}} u_{j}^{2}-2 \frac{f\left(\bar{u}_{j}\right)}{\bar{u}_{j}} u_{j} \phi_{j}-f^{\prime}\left(\bar{u}_{j}\right)\left(u_{j}-\phi_{j}\right)^{2}\right)+o(1) \\
& \leq-\delta \int_{\Omega_{j}} \frac{f\left(\bar{u}_{j}\right)}{\bar{u}_{j}} u_{j}^{2}+o(1) \\
& =-\delta \int_{\Omega_{j}} f\left(u_{j}\right) u_{j}+o(1) \\
& =-\delta \int_{\mathbb{R}_{+}^{N}} f(u) u+o(1),
\end{aligned}
$$


o que implica no resultado.

Note que, como $t_{j}$ é máximo para $\alpha_{j}, \liminf \left|t_{j}-1\right|>0, \liminf \left|\alpha_{j}\left(t_{j}\right)-\alpha_{j}(1)\right|>0$,

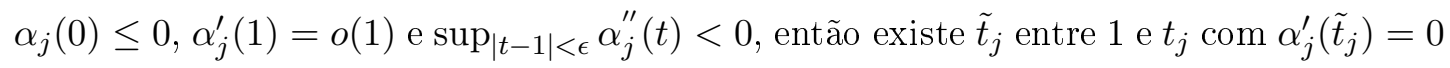
e $\alpha_{j}\left(\tilde{t}_{j}\right)<\alpha_{j}(1)-\rho$ onde a constante $\rho$ independe de $j$.

Assim como no Teorema 2.4.1, denotamos por $\beta_{j}(t)$ a função que coincide com $\alpha_{j}(t)$ em seus pontos críticos, cuja expressão é obtida da mesma forma. Usando o sistema (2.40) segue da mesma forma que no Teorema 2.4.1 que:

$$
\beta_{j}(0) \leq 0 \quad \beta_{j}(1)=\alpha_{j}(1)+o(1)>0 \quad 2 \beta_{j}^{\prime}(1)=-\alpha_{j}^{\prime \prime}(1)+o(1)>0 .
$$

Observando então $\tilde{t}_{j}$ e $(2.41)$, segue que $\beta_{j}^{\prime}$ se anula em algum $\bar{t}_{j}>0 \operatorname{com} \beta_{j}\left(\bar{t}_{j}\right)>0$.

Da mesma forma como no Teorema 3.1, obtemos a propriedade (2.37) que contradiz a existência de $\bar{t}_{j}>0$. Como a contradição veio em supor que $\liminf \left|t_{j}-1\right|>0$ e $\lim \inf \left|\alpha_{j}(t)-\alpha_{j}(1)\right|>0$, então uma delas não ocorre, o que implica no resultado.

\subsection{Resultados de Concentração}

Nessa seção vamos aplicar todos os resultados vistos até aqui nesse capítulo para estabelecer a concentração de soluções do sistema (2.1). Relembremos então o conjunto de hipóteses $(\mathrm{H})$ :

(H) $f, g \in C^{1}(\mathbb{R}), f(0)=g(0)=f^{\prime}(0)=g^{\prime}(0)=0$ e existem $l_{1}, l_{2}>0$ e $p, q>2$ tais que $\frac{1}{p}+\frac{1}{q}>\frac{N-2}{N} \mathrm{e}$

$$
\lim _{|s| \rightarrow \infty} \frac{f^{\prime}(s)}{|s|^{p-2}}=l_{1} \quad \text { e } \quad \lim _{|s| \rightarrow \infty} \frac{g^{\prime}(s)}{|s|^{q-2}}=l_{2} .
$$

Além disso, para algum $\delta>0$ e todo $s \in \mathbb{R}, s \neq 0$,

$$
f(s) s \geq(2+\delta) F(s)>0 \quad \text { e } \quad f^{2}(s) \leq 2 f^{\prime}(s) F(s)
$$

e igualmente para $g$.

Teorema 2.5.1. Seja o sistema (2.1), ou seja, o seguinte sistema:

$$
\left\{\begin{array}{rll}
-\epsilon^{2} \Delta u+u & =g(v) & \text { em } \Omega \\
-\epsilon^{2} \Delta v+v & =f(u) & \text { em } \Omega \\
\frac{\partial u}{\partial n}=\frac{\partial v}{\partial n} & =0 & \text { sobre } \partial \Omega
\end{array},\right.
$$

onde $f, g$ satisfazem o conjunto de hipóteses $(H)$. Então existe $\epsilon_{0}>0$ tal que, para todo $0<\epsilon<\epsilon_{0}$ o sistema (2.1) possui solução $C^{2}(\bar{\Omega})$ positiva não constante $\left(u_{\epsilon}, v_{\epsilon}\right)$. Vale ainda a seguinte estimativa para o indice de Morse relativo:

$$
m\left(u_{\epsilon}, v_{\epsilon}\right) \leq 1
$$


Demonstração. Vamos fazer o seguinte truncamento nas não linearidades:

$$
f(s)=g(s)=0 \quad \forall s \leq 0 .
$$

Devido ao Teorema 2.2.1 podemos supor, sem perda de generalidade, que $2<p=q<2^{*}$. Vamos agora utilizar o conjunto de hipóteses para mostrar que o funcional energia $I$ satisfaz as condições geométricas e todas as hipóteses do Teorema 1.3.2.

- I satisfaz a condição de Palais-Smale.

De fato, mostremos primeiramente que, se $\left(u_{n}, v_{n}\right) \in E$ é uma sequência tal que $\left|I\left(u_{n}, v_{n}\right)\right|<K$ e $I^{\prime}\left(u_{n}, v_{n}\right) \rightarrow 0$, então $\left(u_{n}, v_{n}\right)$ é limitada em $E$. Como

$$
2 I\left(u_{n}, v_{n}\right)=I^{\prime}\left(u_{n}, v_{n}\right)\left(u_{n}, v_{n}\right)+\int_{\Omega}\left(f\left(u_{n}\right) u_{n}-2 F\left(u_{n}\right)\right)+\int_{\Omega}\left(g\left(v_{n}\right) v_{n}-2 G\left(v_{n}\right)\right),
$$

então pela hipótese $(\mathrm{H})$

$$
\int_{\Omega}\left(f\left(u_{n}\right) u_{n}+g\left(v_{n}\right) v_{n}\right) \leq C+\mu_{n}\left(\left\|u_{n}\right\|+\left\|v_{n}\right\|\right)
$$

onde $\mu_{n} \rightarrow 0$. Ainda por $(\mathrm{H})$, dado $\delta>0$, existe $C(\delta)>0$ tal que $F(s) \leq$ $-\delta|s|^{2}+C(\delta)|s|^{p}, \forall s \in \mathbb{R}$ e analogamente para $G(s)$. Dessa forma

$$
\int_{\Omega}\left(\left|u_{n}\right|^{p}+\left|v_{n}\right|^{p}\right) \leq \delta \int_{\Omega}\left(\left|u_{n}\right|^{2}+\left|v_{n}\right|^{2}\right)+C(\delta)+C \mu_{n}\left(\left\|u_{n}\right\|+\left\|v_{n}\right\|\right) .
$$

Como

$$
\left\|u_{n}\right\|^{2}+\left\|v_{n}\right\|^{2}=I^{\prime}\left(u_{n}, v_{n}\right)\left(v_{n}, u_{n}\right)+\int_{\Omega}\left(f\left(u_{n}\right) v_{n}+g\left(v_{n}\right) u_{n}\right),
$$

segue por $(\mathrm{H})$ que

$$
\left\|u_{n}\right\|^{2}+\left\|v_{n}\right\|^{2} \leq \delta \int_{\Omega}\left(\left|u_{n}\right|^{2}+\left|v_{n}\right|^{2}\right)+C(\delta) \int_{\Omega}\left(\left|u_{n}\right|^{p-1}\left|v_{n}\right|+\left|v_{n}\right|^{p-1}\left|u_{n}\right|\right)+\mu_{n}\left(\left\|u_{n}\right\|+\left\|v_{n}\right\|\right) .
$$

Usando então a Desigualdade de Young com $p$ e $p^{\prime}$, obtemos

$$
\begin{gathered}
\left\|u_{n}\right\|^{2}+\left\|v_{n}\right\|^{2} \leq \quad \mu_{n}\left(\left\|u_{n}\right\|+\left\|v_{n}\right\|\right)+\delta \int_{\Omega}\left(\left|u_{n}\right|^{2}+\left|v_{n}\right|^{2}\right)+ \\
C(\delta) \int_{\Omega}\left(\left|u_{n}\right|^{p}+\left|v_{n}\right|^{p}\right) .
\end{gathered}
$$

Usando agora (2.45) e (2.46), temos:

$$
\left\|u_{n}\right\|^{2}+\left\|v_{n}\right\|^{2} \leq \delta \int_{\Omega}\left(\left|u_{n}\right|^{2}+\left|v_{n}\right|^{2}\right)+C(\delta)+\mu_{n}\left(\left\|u_{n}\right\|+\left\|v_{n}\right\|\right),
$$

o que implica que

$$
\left\|u_{n}\right\|+\left\|v_{n}\right\| \leq C
$$

Mostremos agora que a sequência $\left(u_{n}, v_{n}\right)$ converge a menos de subsequência. 
Como $u_{n}$ e $v_{n}$ são limitadas em $H^{1}(\Omega)$, podemos supor sem perda de generalidade que $u_{n}$ e $v_{n}$ convergem fracamente respectivamente para $u$ e $v$ em $H^{1}(\Omega)$ e fortemente para $u$ e $v$ em $L^{p}(\Omega)$. Observando que

$$
I^{\prime}\left(u_{n}, v_{n}\right)\left(0, u_{n}\right)=\left\|u_{n}\right\|^{2}-\int_{\Omega} g\left(v_{n}\right) u_{n}
$$

pelo Teorema da Convergência Dominada de Lebesgue,

$$
\lim _{n \rightarrow \infty}\left\|u_{n}\right\|^{2}=\lim _{n \rightarrow \infty} \int g\left(v_{n}\right) u_{n}=\int g(v) u
$$

Por outro lado, como

$$
I^{\prime}\left(u_{n}, v_{n}\right)(0, u)=\int_{\Omega}\left[\left\langle\nabla u_{n}, \nabla u\right\rangle+u_{n} u\right]-\int_{\Omega} g\left(v_{n}\right) u
$$

usando a convergência fraca e novamente o Teorema da Convergência Dominada de Lebesgue, obtemos

$$
\|u\|^{2}=\int_{\Omega} g(v) u .
$$

Combinando (2.47) e (2.48), concluímos que

$$
\lim _{n \rightarrow \infty}\left\|u_{n}\right\|^{2}=\|u\|^{2}
$$

e analogamente para $v$. Portanto

$$
\left\|u_{n}-u\right\|^{2}=\left\|u_{n}\right\|^{2}+\|u\|^{2}-2 \int\left(\left\langle\nabla u_{n}, \nabla u\right\rangle+u_{n} u\right) \longrightarrow 0
$$

e analogamente para $v$. Logo,

$$
\left\|\left(u_{n}, v_{n}\right)-(u, v)\right\|_{E}^{2}=\left\|u_{n}-u\right\|^{2}+\left\|v_{n}-v\right\|^{2} \longrightarrow 0 .
$$

- $I \leq 0$ em $E^{-}$e para todo $e_{1}, e_{2}>0 \operatorname{com} e=\left(e_{1}, e_{2}\right) \in E$, existe um $R(\epsilon)>0$ suficientemente grande de maneira que

$$
\sup _{\left(E^{-} \oplus \mathbb{R}^{+} e\right) \cap \partial B_{R}(0)} I \leq 0 .
$$

De fato, seja $(\phi,-\phi) \in E^{-}$, então

$$
I(\phi,-\phi)=\int_{\Omega}\left(-\epsilon^{2}|\nabla \phi|^{2}-\phi^{2}-F(\phi)-G(-\phi)\right) \leq 0 .
$$

Seja agora $\left(\phi+\lambda e_{1},-\phi+\lambda e_{2}\right) \in E^{-} \oplus \mathbb{R}^{+} e$, então a prova de que $\sup _{\left(E^{-} \oplus \mathbb{R}^{+} e\right) \cap \partial B_{R}} I \leq 0$ segue como no início da demonstração do Teorema 2.4.1. 
- $I \geq \rho>0$ em $E^{+} \cap \partial B_{r}(0)$ para valores pequenos de $r>0$ e $\rho>0$. Com efeito, seja $(\phi, \phi) \in E^{+} \cap \partial B_{r}(0)$, então usando (2.4) da hipótese $(\mathrm{H})$ dado $\tau>0$, existe constante $C(\tau)>0$, tal que

$$
\begin{aligned}
I(\phi, \phi) & =\int_{\Omega}\left(\epsilon^{2}|\nabla \phi|^{2}+|\phi|^{2}-F(\phi)-G(\phi)\right) \\
& \geq \int_{\Omega}\left(\epsilon^{2}|\nabla \phi|^{2}+|\phi|^{2}-\frac{f(\phi) \phi}{2+\delta}-\frac{g(\phi) \phi}{2+\delta}\right) \\
& \geq \int_{\Omega}\left\{\epsilon^{2}|\nabla \phi|^{2}+|\phi|^{2}-\frac{2 \tau}{2+\delta}|\phi|^{2}-\left.C(\tau \mid) \phi\right|^{p}-C(\tau)|\phi|^{q}\right\} \\
& \geq\|\phi\|^{2}-C \tau\|\phi\|^{2}-C(\tau)\|\phi\|^{p}-C(\tau)\|\phi\|^{q} \\
& \geq r^{2}\left\{1-C \tau-C r^{p-2}-C r^{q-2}\right\}>0,
\end{aligned}
$$

para valores suficientemente pequenos de $r>0$.

Denotando então

$$
S=E^{+} \cap \partial B_{r} \quad \text { e } \quad Q=\left(E^{-} \cap B_{R}(0)\right) \cup\left(\left(E^{-} \oplus \mathbb{R}^{+} e\right) \cap B_{R}(0)\right),
$$

vale o seguinte:

$$
\sup _{\partial Q} I \leq 0<\rho-\delta<\rho \leq \inf _{S} I \quad \text { e } \quad \sup _{Q} I<\infty,
$$

para um $\delta$ suficientemente pequeno. Pelo Teorema 1.3.2, segue que $I$ tem um ponto crítico

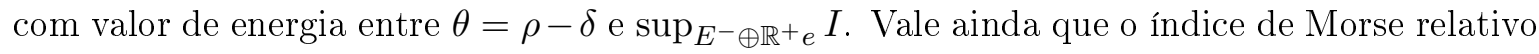
de tal solução é $\leq 1$.

Seja agora $\mathcal{N}=\left\{z \in E ; I^{\prime}(z)=0 \mathrm{e} I(z) \geq \theta\right\}$. Como $I$ satisfaz a condição de Palais-Smale, existe $\left(u_{\epsilon}, v_{\epsilon}\right) \in \mathcal{N}$ tal que

$$
I\left(u_{\epsilon}, v_{\epsilon}\right)=\inf _{\mathcal{N}} I
$$

Como $f(s)=g(s)=0$ para valores negativos de $s$, segue que $u_{\epsilon}, v_{\epsilon}>0$ em $\Omega$. Dessa forma, aplicando novamente o Teorema 1.3.2 a $I$ com $e=\left(u_{\epsilon}, v_{\epsilon}\right)$, teremos que existe um ponto crítico de $I$ com nível de energia entre $\theta$ e $\sup _{E^{-} \oplus \mathbb{R}^{+}\left(u_{\epsilon}, v_{\epsilon}\right)} I$ e índice de Morse relativo $\leq 1$. Pelo Teorema 2.4.1 temos a seguinte estimativa para a energia de $\left(u_{\epsilon}, v_{\epsilon}\right)$, $I\left(u_{\epsilon}, v_{\epsilon}\right)=\sup _{E^{-} \oplus \mathbb{R}^{+}\left(u_{\epsilon}, v_{\epsilon}\right)} I$. Logo, uma vez que $I\left(u_{\epsilon}, v_{\epsilon}\right)=\inf _{\mathcal{N}} I$, segue que a solução $\left(u_{\epsilon}, v_{\epsilon}\right)$ é a proveniente do Teorema 1.3 .2 e portanto $m\left(u_{\epsilon}, v_{\epsilon}\right) \leq 1$.

Resta apenas mostrar que as soluções $\left(u_{\epsilon}, v_{\epsilon}\right)$ são não constantes para valores suficientemente pequenos de $\epsilon$. Isto segue do fato de que para soluções constantes e valores de $\epsilon$ suficientemente pequenos, o índice de Morse não satisfaz a estimativa $m\left(u_{\epsilon}, v_{\epsilon}\right) \leq 1$. De fato, seja $\left(u_{\epsilon}, v_{\epsilon}\right)$ solução constante do sistema (2.1). Então, $\left(u_{\epsilon}, v_{\epsilon}\right)$ satisfaz o seguinte sistema:

$$
\left\{\begin{array}{l}
u=g(v) \text { em } \Omega \\
v=f(u) \text { em } \Omega
\end{array} .\right.
$$

Por definição,

$$
m_{E^{+} \oplus E^{-}}\left(u_{\epsilon}, v_{\epsilon}\right)=\operatorname{dim}_{E^{-}} V=\operatorname{dim}\left(V \cap\left(E^{-}\right)^{\perp}\right)-\operatorname{dim}\left(V^{\perp} \cap E^{-}\right) .
$$


Porém $\operatorname{dim}\left(V^{\perp} \cap E^{-}\right)=0$, uma vez que $V^{\perp} \cap E^{-}=\emptyset$. Note também que se $(\phi, \phi) \in\left(E^{-}\right)^{\perp}$, então:

$I^{\prime \prime}\left(u_{\epsilon}, v_{\epsilon}\right)(\phi, \phi)^{2}=\int_{\Omega}\left(2 \epsilon^{2}|\nabla \phi|^{2}+2 \phi^{2}-f^{\prime}\left(u_{\epsilon}\right) \phi^{2}-g^{\prime}(v) \phi^{2}\right) \leq \int_{\Omega}\left(2 \epsilon^{2}|\nabla \phi|^{2}-2 \delta \phi^{2}\right) \leq 0$,

para valores de $\epsilon$ suficientemente pequenos. Logo $(\phi, \phi) \in V$, portanto, tomando $(\phi, \phi),(\psi, \psi) \in\left(E^{-}\right)^{\perp}$ com suporte disjuntos, $m\left(u_{\epsilon}, v_{\epsilon}\right) \geq 2$.

O teorema seguinte mostra que as soluções obtidas no teorema anterior apresentam a característica de concentração em torno de um ponto de máximo comum para as duas funções, que está localizado na fronteira do domínio $\Omega$, quando $\epsilon \rightarrow 0$.

Teorema 2.5.2. Considere o sistema (2.1) sob o conjunto de hipóteses $(H)$ e as soluções obtidas no Teorema 2.5.1. Então, quando $\epsilon \rightarrow 0$, as soluções $\left(u_{\epsilon}, v_{\epsilon}\right)$ atingem seus valores máximos em um único e comum ponto $x_{\epsilon} \in \partial \Omega$.

Demonstração. Seja $x_{\epsilon}$ ponto de máximo de $u_{\epsilon}$ em $\bar{\Omega}$. Suponhamos por absurdo que

$$
\frac{\operatorname{dist}\left(x_{j}, \partial \Omega\right)}{\epsilon_{j}} \rightarrow \infty
$$

para alguma sequência $x_{j}=x_{\epsilon_{j}}$, onde $\epsilon_{j} \rightarrow 0$. Consideremos agora o reescalonamento das soluções $\tilde{u}_{j}(x)=u_{j}\left(\epsilon_{j} x+x_{j}\right) \rightarrow u, \tilde{v}_{j}(x)=v_{j}\left(\epsilon_{j} x+x_{j}\right) \rightarrow v$. Note que a implicação principal da condição (2.50) é o fato de $\omega=\mathbb{R}^{N}$. Isto será de fundamental importância para o argumento que utilizaremos. Lembremos que as soluções limites $u, v$ são radialmente simétricas com relação a origem.

Pela Proposição 2.2.6, vale a convergência $I_{j}\left(\tilde{u}_{j}, \tilde{v}_{j}\right) \rightarrow I_{\infty}(u, v)$. Logo, utilizando a condição (2.4) temos:

$$
\begin{aligned}
I_{j}\left(\tilde{u}_{j}, \tilde{v}_{j}\right)+o(1) & =\int_{\mathbb{R}^{N}}(\langle\nabla u, \nabla v\rangle+u v-F(u)-G(v)) \\
& =\int_{\mathbb{R}^{N}}\left(\frac{1}{2} f(u) u-F(u)\right)+\int_{\mathbb{R}^{N}}\left(\frac{1}{2} g(v) v-G(v)\right) \\
& =2 \int_{\mathbb{R}_{+}^{N}}\left(\frac{1}{2} f(u) u-F(u)\right)+2 \int_{\mathbb{R}_{+}^{N}}\left(\frac{1}{2} g(v) v-G(v)\right) \\
& >\int_{\mathbb{R}_{+}^{N}}\left(\frac{1}{2} f(u) u-F(u)\right)+\int_{\mathbb{R}_{+}^{N}}\left(\frac{1}{2} g(v) v-G(v)\right) \\
& =\int_{\mathbb{R}_{+}^{N}}(\langle\nabla u, \nabla v\rangle+u v-F(u)-G(v)) .
\end{aligned}
$$

Por outro lado, pela definição do nível de energia $c$, pela construção de $u_{j}, v_{j}$ e pelo Teorema 2.4.4, segue que:

$$
I_{j}\left(\tilde{u}_{j}, \tilde{v}_{j}\right) \leq \int_{\mathbb{R}_{+}^{N}}(\langle\nabla u, \nabla v\rangle+u v-F(u)-G(v))+o(1)
$$


o que contraria a desigualdade estrita anterior. Como a contradição veio da suposição (2.50), segue que:

$$
\operatorname{dist}\left(x_{j}, \partial \Omega\right) \leq C \epsilon_{j}
$$

O resultado segue agora pelo Teorema 2.3.1.

\subsection{Localização dos Pontos de Máximo}

$\mathrm{Na}$ seção anterior provamos que, para valores pequenos de $\epsilon$, os pontos de máximo das funções $u_{\epsilon}$ e $v_{\epsilon}$ solução de (2.1), coincidem e está localizado na fronteira de $\Omega$. Mostraremos nessa seção, seguindo o trabalho [6] de Pistoia e Ramos, que este ponto de máximo localizase no ponto da fronteira que maximiza a sua curvatura média. Para tanto, vamos utilizar estimativas inferiores e superiores para os níveis de energia mínimia em termos da curvatura média da fronteira em diversos pontos.

Visando envolver a curvatura média da fronteira nas estimativas para os níveis de energia mínima que obteremos, vamos raciocinar semelhantemente ao comentário feito após a demonstração do Teorema 2.4.1.

Nessa seção vamos assumir o conjunto de hipóteses $(H)$ enunciado no início da seção anterior. Dado um ponto $P \in \partial \Omega$ arbitrário, podemos fazer uma rotação e uma translação para supor que o ponto $P$ está na origem do sistema de coordenadas e também que o vetor normal exterior a $\partial \Omega$ em $P$ é $(0, \ldots 0,-1)$. Como $\partial \Omega$ é suave, podemos considerá-la localmente como o gráfico de uma função $\alpha: \mathbb{R}^{N-1} \rightarrow \mathbb{R}$ diferenciável, ou seja, $\Omega \cap \mathcal{N}=$ $\left\{\left(x^{\prime}, x\right) \in \mathbb{R}^{N} ; x_{N}>\alpha\left(x^{\prime}\right)\right\} \mathrm{e}$

$\partial \Omega \cap \mathcal{N}=\left\{\left(x^{\prime}, x\right) \in \mathbb{R}^{N} ; x_{N}=\alpha\left(x^{\prime}\right)\right\}$, onde $\mathcal{N}$ é uma vizinhança de $P$. Dessa forma teremos que a função $\alpha$ é tal que:

- $\alpha(0)=0$;

- $\nabla \alpha(0)=0$

Através da função $\alpha$ vamos definir a função $\Phi$ que mostraremos depois se tratar de um difeomorfismo que transforma uma porção de um hiperplano contendo a origem em uma porção da fronteira de $\Omega$ que inclui o ponto $P$. Seja então $\Phi\left(x^{\prime}, x_{N}\right)=\left(x^{\prime}, \alpha\left(x^{\prime}\right)\right)-$ $x_{N} n\left(\left(x^{\prime}, \alpha\left(x^{\prime}\right)\right)\right)$ onde $n(y)$ é o vetor normal exterior a $\partial \Omega$ em $y \in \partial \Omega$. Pela construção de $\alpha$, note que o vetor $n\left(\left(x^{\prime}, \alpha\left(x^{\prime}\right)\right)=\left(\nabla \alpha\left(x^{\prime}\right),-1\right)\right.$. Dessa forma

$$
\Phi\left(x^{\prime}, x_{N}\right)=\left(x^{\prime}-x_{N} \nabla \alpha\left(x^{\prime}\right), \alpha\left(x^{\prime}\right)+x_{N}\right) .
$$

Note então que $D \Phi(0)=I d$, assim, pelo Teorema da Função Inversa, existe uma inversa local de $\Phi$ em $P$ que denotaremos por $\Psi$.

No trabalho [12] os autores mostram que existe uma constante $C=C(P)=\Delta \alpha(0)=$ $\Delta \Phi_{N}(0)$ tal que $\operatorname{det} D(\Phi(x))=1-C x_{N}+O\left(|x|^{2}\right)$ quando $|x| \rightarrow 0$. 
Por definição, a curvatura média de $\partial \Omega$ em $P$ é $H(P)=\frac{C(P)}{N-1}$. Utilizando isso juntamente com a seguinte identidade $\Delta \Psi_{N}(P)=-\Delta \Phi_{N}(0)=-\Delta \alpha(0)$, segue que

$$
\Delta \Psi_{N}(P)=-\Delta \Phi_{N}(0)=-\Delta \alpha(0)=-H(P)(N-1)
$$

Considerando uma sequência $\epsilon_{j} \rightarrow 0$ e a solução $\left(u_{\epsilon_{j}}, v_{\epsilon_{j}}\right)$ do problema (2.1) obtida através do Teorema 2.5.1, seja $\tilde{u}_{j}, \tilde{v}_{j}$ como em (2.25) (que convergem em $C_{l o c}^{2}$, respectivamente, para as funções $u, v \in H^{1}\left(\mathbb{R}_{+}^{N}\right)$ ). De acordo com o Teorema 2.5.2 podemos supor que a sequência de pontos de máximo $x_{j}$ de $u_{\epsilon_{j}}, v_{\epsilon_{j}}$, converge, a menos de subsequência, para um ponto $P_{0} \in \partial \Omega$.

O teorema seguinte estabelecerá uma estimativa superior para a energia das soluções $\left(u_{\epsilon_{j}}, v_{\epsilon_{j}}\right)$, em termos da curvatura média da fronteira calculada sobre um ponto qualquer $P \in \partial \Omega$.

Teorema 2.6.1. Se $P \in \partial \Omega$, então vale a seguinte estimativa:

$$
I\left(u_{\epsilon_{j}}, v_{\epsilon_{j}}\right) \leq \epsilon_{j}^{N}\left[I_{\infty}(u, v)-\epsilon_{j}(N-1) H(P) \gamma+o\left(\epsilon_{j}\right)\right] \quad \text { quando } \epsilon_{j} \rightarrow 0
$$

onde $\gamma$ é uma constante positiva definida por

$$
\gamma=\gamma(f)+\gamma(g)+\frac{1}{2} \int_{\partial \mathbb{R}_{+}^{N}} u v
$$

com

$$
\gamma(f)=\int_{\mathbb{R}_{+}^{N}}\left(\frac{1}{2} f(u) u-F(u)\right) x_{N} d x
$$

e semelhantemente para $\gamma(g)$.

A prova desse teorema seguirá de alguns lemas que serão expostos a seguir e também de algumas observações já feitas no texto.

Por simplicidade de notação, suponhamos que $P=0$. Seja $\Omega_{j}=\frac{1}{\epsilon_{j}} \Omega$ e definamos $u_{j}, v_{j}$ como em (2.39) com $\Psi$ dada como no início dessa seção. Observamos na demonstração do Teorema 2.4.4 que as funções $u_{j}, v_{j}$ satisfazem o sistema (2.40), onde $\mu_{j}(x)$ e $\nu_{j}(x)$ são $o(1)$ em um certo sentido. Na verdade pode-se mostrar que as funções $\mu_{j}, \nu_{j}$ são tais que

$$
\int_{\Omega_{j}} \mu_{j}(x) \phi_{j}(x) d x=\left\|\phi_{j}\right\| O\left(\epsilon_{j}\right) \quad \text { quando } j \rightarrow \infty \text {. }
$$

Vamos assumir a seguinte identidade e utilizá-la para provar o próximo Lema:

$$
\int_{\Omega_{j}}\left(\mu_{j} v_{j}+\nu_{j} u_{j}\right)=-\epsilon_{j} \Delta \alpha(0) \int_{\partial \mathbb{R}_{+}^{N}} u v+o\left(\epsilon_{j}\right) \quad \text { quando } j \rightarrow \infty
$$

Lema 2.6.2. Vale a seguinte identidade:

$$
I_{j}\left(u_{j}, v_{j}\right)=I_{\infty}(u, v)-\epsilon_{j}(N-1) H(P) \gamma+o\left(\epsilon_{j}\right) \quad \text { quando } j \rightarrow \infty .
$$


Demonstração. Na demonstração da Proposição 2.2.6, mostramos que para qualquer $\delta>0$, existe $j_{0} \in \mathbb{N}$ e $R>0$ tais que, para todo $j \geq j_{0}$,

$$
\int_{\Omega_{j} \cap B_{2 R}^{C}}\left(\left|\nabla \tilde{u}_{j}\right|^{2}+\left|\nabla \tilde{v}_{j}\right|^{2}+\tilde{u}_{j}^{2}+\tilde{v}_{j}^{2}+f\left(\tilde{u}_{j}\right) \tilde{u}_{j}+g\left(\tilde{v}_{j}\right) \tilde{v}_{j}\right) \leq \delta .
$$

Utilizando esta desigualdade, mostra-se como em [12] que

$$
\int_{\Omega_{j}}\left(\frac{1}{2} f\left(u_{j}\right) u_{j}-F\left(u_{j}\right)\right)=\int_{\mathbb{R}_{+}^{N}}\left(\frac{1}{2} f(u) u-F(u)\right)-\epsilon_{j} \Delta \alpha(0) \gamma(f)+o\left(\epsilon_{j}\right) .
$$

Multiplicando o sistema (2.40) por $v_{j}$ e $u_{j}$, integrando por partes, utilizando a igualdade acima e (2.53) teremos que:

$$
\begin{aligned}
I_{j}\left(u_{j}, v_{j}\right)= & \int_{\Omega_{j}}\left(\left\langle\nabla u_{j}, \nabla v_{j}\right\rangle+u_{j} v_{j}-F\left(u_{j}\right)-G\left(v_{j}\right)\right) \\
= & \int_{\Omega_{j}}\left(\frac{1}{2} f\left(u_{j}\right) u_{j}-F\left(u_{j}\right)\right)+\int_{\Omega_{j}}\left(\frac{1}{2} g\left(v_{j}\right) v_{j}-G\left(v_{j}\right)\right) \\
& +\frac{1}{2} \int_{\Omega_{j}}\left(\mu_{j} v_{j}+\nu_{j} u_{j}\right) \\
= & \int_{\mathbb{R}_{+}^{N}}\left(\frac{1}{2} f(u) u-F(u)\right)+\int_{\mathbb{R}_{+}^{N}}\left(\frac{1}{2} g(v) v-G(v)\right) \\
& -\epsilon_{j} \Delta \alpha(0)\left(\gamma(f)+\gamma(g)+o\left(\epsilon_{j}\right)\right)+\frac{1}{2} \int_{\Omega_{j}}\left(\mu_{j} v_{j}+\nu_{j} u_{j}\right) \\
= & \int_{\mathbb{R}_{+}^{N}}\left(\frac{1}{2} f(u) u-F(u)\right)+\int_{\mathbb{R}_{+}^{N}}\left(\frac{1}{2} g(v) v-G(v)\right) \\
& -\epsilon_{j} \Delta \alpha(0)\left(\gamma(f)+\gamma(g)+\frac{1}{2} \int_{\partial \mathbb{R}_{+}^{N}} u v\right)+o\left(\epsilon_{j}\right) \\
= & I_{\infty}(u, v)-\epsilon_{j} \Delta \alpha(0) \gamma+o\left(\epsilon_{j}\right),
\end{aligned}
$$

o que prova o Lema.

Vamos agora apresentar a demonstração do Teorema 2.6.1. Esta utilizará uma estimativa muito semelhante, porém mais forte, que a apresentada no Teorema 2.4.4. A argumentação para a demonstração também é bastante parecida com a utilizada na demonstração daquele Teorema.

Demonstração do Teorema 2.6.1. Mostremos inicialmente a seguinte estimativa para o nível de energia da solução $\left(u_{j}, v_{j}\right)$ :

$$
\sup _{E^{-} \oplus \mathbb{R}^{+}\left(u_{j}, v_{j}\right)} I_{j}=I_{j}\left(u_{j}, v_{j}\right)+o\left(\epsilon_{j}\right) \quad \text { quando } j \rightarrow \infty .
$$

Seja $\left\|\phi_{j}\right\|=1$ e $s_{j}\left(u_{j}, v_{j}\right)+t_{j}\left(\phi_{j},-\phi_{j}\right)$ uma sequência de pontos onde o supremo acima é atingido. Pela forma contrapositiva do Lema 2.4.3, vemos que $\left|t_{j}\right|+\left|s_{j}\right| \leq C$. Definamos

$$
\chi_{j}(s, t)=I_{j}\left(s\left(u_{j}, v_{j}\right)+t\left(\phi_{j},-\phi_{j}\right)\right),
$$


de maneira que $\left(s_{j}, t_{j}\right)$ é ponto de máximo para $\chi_{j}$. A idéia da demonstração de $(2.54)$ é utilizar a definição de diferenciabilidade de $\chi_{j}$ em $(1,0)$ e, posteriormente, estimativas em termos de $\epsilon_{j}$ de $\left|s_{j}-1\right|$ e $\left|t_{j}\right|$. Para tanto seguiremos alguns passos.

1. Temos

$$
\lim _{j \rightarrow \infty} s_{j}=1 .
$$

De fato, suponhamos por contradição que exista uma subsequência tal que $\left|s_{j}-1\right| \geq$ $\delta>0$. Isso implica que as seguintes funções são limitadas uniformemente em $j$,

$$
\psi_{j}=\frac{t_{j}}{1-s_{j}}
$$

Definamos agora

$$
\alpha_{j}(s)=I_{j}\left(s\left(u_{j}, v_{j}\right)+(1-s)\left(\psi_{j},-\psi_{j}\right)\right),
$$

e note que essa função tem ponto de máximo em $s=s_{j}$. A discussão que faremos a seguir é muito semelhante a aquela empregada na demonstração do Teorema 2.4.4. Denotemos por $\beta_{j}(s)$ uma função que concorda com $\alpha_{j}$ em seus pontos críticos. Na demonstração do Teorema 2.4.4 mostrou-se que o fato de $\liminf \left|s_{j}-1\right|>0$ implicava na existência de uma constante $\rho>0$ e um ponto $\tilde{s}_{j}$ entre 1 e $s_{j}$ tais que:

$$
\beta_{j}\left(\tilde{s}_{j}\right)<\alpha_{j}(1)-\rho, \beta_{j}(0) \leq 0, \beta_{j}(1)=\alpha_{j}(1)+o(1), \beta_{j}^{\prime}(1)>0
$$

para valores grandes de $j$ e ainda que $\beta_{j}^{\prime}(s) \neq 0$ sempre que $\beta_{j}(s)>0$. Temos agora dois casos a considerar:

- Se $0<s_{j}<1$ então

$$
\beta_{j}(0) \leq 0<\alpha_{j}(1) \leq \alpha_{j}\left(s_{j}\right)=\beta_{j}\left(s_{j}\right)
$$

pois, usando (2.4)

$$
\alpha(1)=I_{j}\left(u_{j}, v_{j}\right) \geq\left(\frac{1}{2}-\frac{1}{2+\delta}\right) \int_{\Omega_{j}}\left(f\left(u_{j}\right) u_{j}+g\left(v_{j}\right) v_{j}\right)+\frac{1}{2} \int_{\Omega_{j}}\left(\mu_{j} v_{j}+\nu_{j} u_{j}\right)>0 .
$$

Temos ainda que

$$
\beta_{j}\left(\tilde{s}_{j}\right)<\alpha_{j}(1)-\rho \leq \alpha_{j}\left(s_{j}\right)-\rho=\beta_{j}\left(s_{j}\right)-\rho<\beta_{j}\left(s_{j}\right),
$$

ou seja

$$
\max \left\{\beta_{j}(0), \beta_{j}\left(\tilde{s}_{j}\right)\right\}<\beta_{j}\left(s_{j}\right) \text { e } s_{j} \in\left(0, \tilde{s}_{j}\right) .
$$

Isso implica que $\beta_{j}^{\prime}$ se anula em algum ponto onde $\beta_{j}>0$, o que contraria o exposto acima.

- Se $1<s_{j}$, então $1<\tilde{s}_{j}<s_{j}$. Logo, para valores de $j$ suficientemente grandes

$$
\beta_{j}\left(\tilde{s}_{j}\right)<\alpha_{j}(1)-\rho<\beta_{j}(1) .
$$

Como $\beta_{j}^{\prime}(1)>0, \beta_{j}^{\prime}$ precisa se anular em algum ponto onde $\beta_{j}>0$, o que contraria novamente o exposto acima. 
Dessa forma, notamos que de fato precisa valer (2.55).

2. Mostremos agora que existe $C>0$ constante tal que

$$
\left|t_{j}\right| \leq C\left(\left|s_{j}-1\right|+\epsilon_{j}\right)
$$

Com efeito, seja $\theta_{j}(t)=\chi_{j}\left(s_{j}, t\right)=I_{j}\left(s_{j}\left(u_{j}, v_{j}\right)+t\left(\phi_{j},-\phi_{j}\right)\right)$. Note então que $\theta_{j}^{\prime}\left(t_{j}\right)=0$, uma vez que $\left(s_{j}, t_{j}\right)$ é ponto de máximo de $\chi_{j}$. Na demonstração do Teorema 2.4.4 mostramos que $\theta_{j}^{\prime \prime}(t) \leq-2$ para todo $t \in \mathbb{R}$ e $j \in \mathbb{N}$.

Mostremos agora que

$$
\left|\theta_{j}^{\prime}(0)\right| \leq C\left(\left|s_{j}-1\right|+\epsilon_{j}\right)
$$

De fato, calculando $\theta_{j}^{\prime}(0)$, multiplicando as equações do sistema (2.40) por $\phi$ e $-\phi$, respectivamente e integrando por partes, teremos que:

$$
\begin{aligned}
\theta_{j}^{\prime}(0)= & \left(s_{j}-1\right) \int g\left(v_{j}\right) \phi_{j}-\left(s_{j}-1\right) \int f\left(u_{j}\right) \phi_{j} \\
& +\int\left(g\left(v_{j}\right)-g\left(s_{j} v_{j}\right)\right) \phi_{j}-\int\left(f\left(u_{j}\right)-f\left(s_{j} u_{j}\right)\right) \phi_{j} \\
& +s_{j} \int\left(\mu_{j} \phi_{j}-\nu_{j} \phi_{j}\right) .
\end{aligned}
$$

Usando Hölder, a hipótese $(H)$ e o fato de $\left\|\phi_{j}\right\|=1$, verificamos que

$$
\left(s_{j}-1\right) \int f\left(u_{j}\right) \phi_{j}=O\left(\left|s_{j}-1\right|\right)
$$

e semelhantemente para $g$. Pela igualdade (2.52), segue que

$$
s_{j} \int\left(\mu_{j} \phi_{j}-\nu_{j} \phi_{j}\right)=O\left(\epsilon_{j}\right)
$$

Para a terceira e quarta integrais, note que pela hipótese $(\mathrm{H})$,

$$
\left|f^{\prime}(s)\right| \leq C\left(|s|+|s|^{p-2}\right) \leq C\left(|s|+|s|^{p-1}\right) .
$$

Usando então o Teorema do Valor Médio, as desigualdades de Hölder (com $p$ e $p^{\prime}=\frac{p}{p-1}$ ), Minkowski e o Teorema de Imersão de Sobolev (ver [13], cap.7), segue que:

$$
\begin{aligned}
\int_{\Omega_{j}}\left|f\left(u_{j}\right)-f\left(s_{j} u_{j}\right)\right|\left|\phi_{j}\right| & \leq C\left|s_{j}-1\right|\left\|f^{\prime}(s)\right\|_{L^{p^{\prime}}}\left\|\phi_{j}\right\|_{L^{p}} \\
& \leq C\left|s_{j}-1\right|\left(\int_{\Omega_{j}}\left(|s|+|s|^{p-1}\right)^{\frac{p}{p-1}}\right)^{\frac{p-1}{p}} \\
& \leq C\left|s_{j}-1\right|\|s\|_{L^{p^{\prime}}}\left(\|s\|_{L^{p^{\prime}}}+\|s\|_{L^{p}}^{p}-1\right) \\
& \leq C\left|s_{j}-1\right|,
\end{aligned}
$$

onde $s \in H^{1}\left(\Omega_{j}\right)$. Logo vale (2.57). 
Usando agora o Teorema do Valor Médio e (2.57), segue que

$$
\theta_{j}^{\prime}\left(t_{j}\right)-\theta_{j}^{\prime}(0)=t_{j} \theta_{j}^{\prime \prime}\left(s t_{j}\right) \leq t_{j}(-2) \Rightarrow t_{j} \leq \frac{1}{2} \theta_{j}^{\prime}(0) \leq C\left(\left|s_{j}-1\right|+\epsilon_{j}\right),
$$

o que estabelece (2.56).

3. Mostremos agora que existe $C>0$ tal que, para todo $j \in \mathbb{N}$

$$
\left|s_{j}-1\right|+\left|t_{j}\right| \leq C \epsilon_{j}
$$

Podemos supor, sem perda de generalidade, que $\epsilon_{j} \leq\left|s_{j}-1\right|$, pois caso contrário, como $\epsilon_{j} \rightarrow 0$, podemos considerar uma subsequência que satisfaça essa condição. Consideremos então novamente a função $\alpha_{j}$ tal como no primeiro passo. Como $\left(u_{j}, v_{j}\right)$ satisfaz o sistema (2.40), utilizando (2.52), segue que

$$
\begin{aligned}
\alpha_{j}^{\prime}(1) & =I_{j}^{\prime}\left(u_{j}, v_{j}\right)\left(u_{j}-\psi_{j}, v_{j}+\psi_{j}\right) \\
& =\int_{\Omega_{j}}\left(\mu_{j}\left(v_{j}+\psi_{j}\right)+\nu_{j}\left(u_{j}-\psi_{j}\right)\right)=O\left(\epsilon_{j}\right) .
\end{aligned}
$$

Tal como no Teorema 2.4.4, podemos mostrar que $\sup _{|s-1|<\delta} \alpha_{j}^{\prime \prime}(s)<0$ para $\delta>0$ suficientemente pequeno. Como $s_{j} \rightarrow 1$, para qualquer $s$ entre 1 e $s_{j}$, vale

$$
\left|\alpha^{\prime \prime}(s)\right| \geq \rho>0
$$

Utilizando mais uma vez o Teorema do Valor Médio e (2.59) e (2.60), segue que

$$
\alpha_{j}^{\prime}\left(s_{j}\right)-\alpha_{j}^{\prime}(0) \leq\left|s_{j}-1\right| \alpha_{j}^{\prime \prime}(s)<\left|s_{j}-1\right|(-\rho) \Rightarrow\left|s_{j}-1\right| \leq C \epsilon_{j},
$$

onde $s$ é um valor intermediário entre 1 e $s_{j}$. Por (2.56), temos

$$
\left|t_{j}\right| \leq C\left(\left|s_{j}-1\right|+\epsilon_{j}\right) \leq C\left|s_{j}-1\right| \leq C \epsilon_{j},
$$

o que mostra o desejado.

4. Relembremos a definição de $\chi_{j}$ :

$$
\chi_{j}(s, t)=I_{j}\left(s\left(u_{j}, v_{j}\right)+t\left(\phi_{j},-\phi_{j}\right)\right) .
$$

Então

$$
\frac{\partial \chi_{j}}{\partial s}(1,0)=I^{\prime}\left(u_{j}, v_{j}\right)\left(u_{j}, v_{j}\right)=\int_{\Omega_{j}}\left(\nu_{j} u_{j}+\mu_{j} v_{j}\right)=o(1),
$$

enquanto que

$$
\frac{\partial \chi_{j}}{\partial t}(1,0)=I^{\prime}\left(u_{j}, v_{j}\right)\left(\phi_{j},-\phi_{j}\right)=\int_{\Omega_{j}}\left(\nu_{j} \phi_{j}-\mu_{j} \phi_{j}\right)=o(1) .
$$


Dessa forma, pela definição de diferenciabilidade de $\chi_{j}$ em $(1,0)$, segue que:

$$
\begin{aligned}
\chi_{j}\left(s_{j}, t_{j}\right)-\chi_{j}(1,0) & =\nabla \chi_{j}(1,0)\left(s_{j}-1, t_{j}\right)+o\left(\left|s_{j}-1\right|+\left|t_{j}\right|\right) \\
& =\chi_{j}(1,0)+\left(s_{j}-1\right) o(1)+t_{j} o(1)+o\left(\left|s_{j}-1\right|+\left|t_{j}\right|\right) .
\end{aligned}
$$

Então substituindo os valores de $\chi_{j}$, segue por (2.58) que

$$
I_{j}\left(s_{j}\left(u_{j}, v_{j}\right)=I\left(u_{j}, v_{j}\right)+o\left(\epsilon_{j}\right),\right.
$$

o que implica na estimativa (2.54).

Agora que foi mostrada a estimativa (2.54), podemos finalizar a prova. Pelo final da demonstração do Teorema 2.5.2,

$$
I_{j}\left(\tilde{u}_{j}, \tilde{v}_{j}\right) \leq \sup _{E^{-} \oplus \mathbb{R}^{+}\left(u_{j}, v_{j}\right)} I_{j}
$$

Então, usando o Lema 2.6.2, a estimativa (2.54) e o Teorema de Mudança de Variáveis em integrais

$$
\begin{aligned}
& I\left(u_{\epsilon_{j}}, v_{\epsilon_{j}}\right)=\epsilon_{j}^{N}\left(I_{j}\left(\tilde{u}_{j}, \tilde{v}_{j}\right)\right)
\end{aligned}
$$

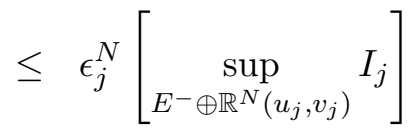

$$
\begin{aligned}
& =\epsilon_{j}^{N}\left[I_{j}\left(u_{j}, v_{j}\right)+o\left(\epsilon_{j}\right)\right] \\
& \leq \epsilon_{j}^{N}\left[I_{\infty}(u, v)-\epsilon_{j}(N-1) H(P) \gamma+o\left(\epsilon_{j}\right)\right] \text {, }
\end{aligned}
$$

quando $\epsilon_{j} \rightarrow 0$.

Agora que obtemos uma estimativa superior para o nível de energia da solução $\left(u_{\epsilon_{j}}, v_{\epsilon_{j}}\right)$ em termos da curvatura média da fronteira de $\Omega$ em um ponto arbitrário $P$, nos resta obter uma estimativa inferior do nível de energia de $\left(u_{\epsilon_{j}}, v_{\epsilon_{j}}\right)$ em termos da curvatura média da fronteira no ponto $P_{0}=\lim _{j \rightarrow \infty} x_{\epsilon_{j}}$, onde $x_{\epsilon_{j}}$ é o ponto de máximo de $u_{\epsilon_{j}}$ e $v_{\epsilon_{j}}$. Uma vez que a argumentação para a obtenção de tal estimativa é bastante semelhante àquela utilizada até agora nessa seção, vamos então assumir o seguinte Teorema.

Teorema 2.6.3. Vale a seguinte estimativa:

$$
I\left(u_{\epsilon_{j}}, v_{\epsilon_{j}}\right) \geq \epsilon_{j}^{N}\left[I_{\infty}(u, v)-\epsilon_{j}(N-1) H\left(P_{0}\right) \gamma+o\left(\epsilon_{j}\right)\right]
$$

quando $\epsilon_{j} \rightarrow 0$, onde $u$ e $v$ são os limites $C_{l o c}^{2}$ das funçôes reescalonadas $\tilde{u}_{j}$ e $\tilde{v}_{j}$, respectivamente.

Munido dessas duas estimativas, podemos agora enunciar e provar o teorema que garantirá que as soluções $u_{\epsilon_{j}}$ e $v_{\epsilon_{j}}$ do sistema (2.1) se concentram em torno do ponto da fronteira de $\Omega$ que maximiza a sua curvatura média. 
Teorema 2.6.4. Seja $P_{0}=\lim _{\epsilon \rightarrow 0} x_{\epsilon}$, onde $x_{\epsilon}$ é como no Teorema 2.5.2. Então

$$
H\left(P_{0}\right)=\max _{P \in \partial \Omega} H(P) .
$$

Demonstração. Pelos Teoremas 2.6.1 e 2.6.3, segue que

$$
H(P) \leq H\left(P_{0}\right)
$$

onde $P \in \partial \Omega$ é arbitrário. Portanto temos o resultado. 


\section{Referências Bibliográficas}

[1] Abbondandolo, A., Felmer, P., Molina, J. - An estimate on the relative Morse index for strongly indefinite functionals, Electron. J. Diff. Equations, Conf. 06, 1-11 $(2001)$

[2] Angenent, S., VAn Der Vorst, R. - A priori bounds and renormalized Morse indices of solutions fo an elliptic system, Ann. Inst. H. Poincaré Anal. Non Linéaire $17,277-306(2000)$

[3] Busca, J., Sirakov, B. - Symmetry results for semilinear elliptic systems in the whole space, Journal of Differential Equations 163, 41 - 56 (2000)

[4] Del Pino, M., Felmer, P. - Spike-layered solutions of singularly perturbed elliptic problems in a degenerate setting, Indiana Univ. math J. 48, 883 - 898 (1999)

[5] Marino, A., Prodi, G. - Metodi perturbativi nella teoria di Morse, Boll. Un. Mat. Ital. A (7) 4, 1 - 32 (1975)

[6] Pistoia, A., Ramos, M. - Locating the peaks of the least energy solutions to an elliptic system with Neumann boundary conditions, Journal of Differential Equations 201, 160 - 176 (2004)

[7] Rabinowitz, P. - Minimax methods in CRitical Point theory With applications to Differential equations, Regional Conference Series in Mathematics at the Un. of Miami 65 (1984)

[8] Ramos, M., Yang, J. - Spike-layered solutions for an elliptic system with Neumann boundary conditions, Transactions of the American Mathematical Society, 357, 3265 - $3284(2005)$ 
[9] Ramos, M., Terracini, S., Troestler, C. - Superlinear indefinite elliptic problems and Pohozaev type identities, Journal of Functional Analysis 159, 596 $628(1998)$

[10] Schwartz, J. - Nonlinear Functional Analysis, Gordon (1969)

[11] SiRAKov, B. - On the existence of solutions of Hamiltonian system in $\mathbb{R}^{N}$, Adv. Diff. Eq. 5, 1445 - 1464 (2000)

[12] Ni, W., TAKAGI, I. - On the shape of least-energy solutions to a semilinear Neumann problem, Comm. Pure Appl. Mathematics 44, 819 - 851 (1991)

[13] Trudinger, N., Gilbarg, D. - Elliptic partial differential equations of second order, Springer (2001)

[14] Willem, M. - Minimax theorems, Birkhäuser (1996) 
